\title{
LOCAL SMOOTHING OF FOURIER INTEGRAL OPERATORS AND CARLESON-SJÖLIN ESTIMATES
}

\author{
GERD MOCKENHAUPT, ANDREAS SEEGER, AND CHRISTOPHER D. SOGGE
}

\section{INTRODUCTION}

The purpose of this paper is twofold. First, if $Y$ and $Z$ are smooth paracompact manifolds of dimensions $n \geqslant 2$ and $n+1$, respectively, we shall prove local regularity theorems for a certain class of Fourier integral operators $\mathscr{F} \in I^{\mu}(Z, Y ; \mathscr{C})$ which naturally arise in the study of wave equations. Secondly, we want to apply these estimates to prove versions of the Carleson-Sjölin theorem on compact two-dimensional manifolds with periodic geodesic flow.

The operators we shall study satisfy the curvature condition introduced in [32]. The main example occurs when $Z=Y \times \mathbb{R}$ and $\mathscr{F}$ is the solution operator for the Cauchy problem for the wave equation

$$
\begin{gathered}
\left((\partial / \partial t)^{2}-\Delta\right) \mathscr{F} f=0, \\
\left.\mathscr{F} f\right|_{t=0}=f,\left.\quad(\partial / \partial t) \mathscr{F} f\right|_{t=0}=0 .
\end{gathered}
$$

Here $\Delta$ is the Laplace-Beltrami operator associated to some metric $g$ on $Y$, and $L_{\alpha}^{p}$ denotes the usual Sobolev space. Then the solution operator $\mathscr{F}_{t}$ at fixed time $t$, is bounded from $L_{\text {comp }}^{p}(Y)$ to $L_{\text {loc }, \alpha}^{p}(Y)$ if $\alpha \leqslant-(n-1)|1 / 2-1 / p|$. This is a special case of a result for general hyperbolic equations in [28]. It is sharp for all but a discrete set of times $t$. One of our chief results implies that, if $2<p<\infty$, then there is a $\delta=\delta(p, n)>0$ so that $\mathscr{F}$ is bounded from $L_{\text {comp }}^{p}(Y)$ to $L_{\text {loc }, \alpha}^{p}(Y \times \mathbb{R})$ if $\alpha \leqslant-(n-1)|1 / 2-1 / p|+\delta$. Such a result is called $L^{p} \rightarrow L^{p}$ local smoothing of order $\delta$. It is not hard to see that there can never be $L^{p} \rightarrow L^{p}$ local smoothing when $p=\infty$ or $p \leqslant 2$.

It was conjectured in [32] that for $p \geqslant 2 n /(n-1)$ there should always be local smoothing of order $<1 / p$. Although we are not close to proving this conjecture in any dimension, our estimates give a simplified proof of the variable coefficient version of Bourgain's circular maximal theorem $[4,32]$ and also improve Stein's spherical maximal theorem [35] for exponents $2<p<\infty$.

Received by the editors June 13, 1991.

1991 Mathematics Subject Classification. Primary 35P10, 42B25, 58G15.

Key words and phrases. $L^{p}$ space-time regularity for the wave equation, maximal averages, variable coefficient Kakeya maximal functions, Riesz means.

The authors were supported in part by the National Science Foundation. The first author was also supported by a Fritz Thyssen Stiftung fellowship. 
Proving the sharp $L^{p} \rightarrow L^{p}$ local smoothing estimates appears to be very difficult. Our methods do, however, allow us to obtain some sharp local smoothing estimates for Fourier integral operators of type $(1 / 2,1 / 2)$.

It turns out that it is harder to prove $L^{p} \rightarrow L^{p}$ local smoothing in two or three dimensions. We shall adapt the arguments used in the translation invariant case [24]. One of the main tools we shall need to use is a variable coefficient version of Córdoba's Kakeya maximal theorem [12]. A special case of our result is the following: Let $(Y, g)$ be a compact two-dimensional Riemannian manifold. Then, if $0<T<\infty$ is fixed, we let $R_{y, \theta}^{\delta}$ be the tubular neighborhood of width $0<\delta<1 / 2$ around the geodesic of length $T$ starting at $y \in Y$ in the direction $\theta \in T_{y} Y \backslash 0$. So $R_{y, \theta}^{\delta}$ should be thought of as a "geodesic rectangle" of thickness $\delta$ in the direction $\theta$. Then the following maximal inequality holds:

$$
\left\|\sup _{\theta} \frac{1}{\operatorname{Vol}\left(R_{y, \theta}^{\delta}\right)} \int_{R_{y, \theta}^{\delta}}|f| \mathrm{d} \operatorname{Vol}\right\|_{L^{2}(Y)} \leqslant C_{T}|\log \delta|^{1 / 2}\|f\|_{L^{2}(Y)} .
$$

The constants of course depend on $T$, but we make no restrictions on $T$; specifically, we do not require $T$ to be smaller than the injectivity radius of $(Y, g)$. As in the Euclidean case, the bounds in (1.2) are sharp.

It is easier to prove sharp $L^{2} \rightarrow L^{p}$ local smoothing estimates. If we consider solutions of the Cauchy problem for the wave equation, these say that when $2(n+1) /(n-1) \leqslant p<\infty$ there is a gain of regularity of order $1 / p$ if one compares the $L_{\text {comp }}^{2}(Y) \rightarrow L_{\mathrm{loc}, \alpha}^{p}(Y)$ mapping properties to the $L_{\text {comp }}^{2}(Y) \rightarrow L_{\text {loc }, \alpha}^{p}(Y \times \mathbb{R})$ mapping properties of the solution operator. This result is related to Strichartz's $L^{2}$ restriction theorem for the Fourier transform [39] and should be thought of as a variable coefficient version of his result. We shall further improve on this by examining the $L_{\text {comp }}^{2}(Y) \rightarrow L_{\mathrm{loc}, \alpha}^{p}\left(Y ; L^{2}(I)\right)$ mapping properties of the solution operator. Here $I$ is a compact interval on the real line. Both of these estimates are proved by adapting arguments of Tomas and Stein (see [36]).

We shall see that our curvature condition is the natural homogeneous version of the Carleson-Sjölin condition [9, 21, 36] for nonhomogeneous phase functions. The second main purpose of this paper is to prove a version of the Carleson-Sjölin theorem. Our results concern compact two-dimensional Riemannian manifolds $(M, g)$ with periodic geodesic flow. We consider positive selfadjoint differential operators $-\Delta+V$ where $-\Delta$ is the Laplace-Beltrami operator and $V$ is a smooth potential. Under the assumption that the geodesic flow is periodic with minimal period $\Pi$ it is known (see $[40 ; 11 ; 20$, Chapter XXIX]) that the eigenvalues $\lambda_{j}$ of $-\Delta+V$ are clustered around the sequence $\sigma_{k}^{2}$ where $\sigma_{k}$ is the arithmetic progression $\sigma_{k}=(2 \pi / \Pi)(k+\kappa / 4)$ and $\kappa$ is the number of conjugate points in the interval $(0, \Pi]$ (counted with multiplicity). If $f \rightarrow e_{j}(f)$ is the projection onto the eigenspace for $\lambda_{j}$, and if $I_{k}=\left(\sigma_{k}-\pi / \Pi, \sigma_{k}+\pi / \Pi\right]$, we define the projection operator corresponding 
to a cluster of eigenvalues by

$$
E_{k} f=\sum_{\sqrt{\lambda_{j}} \in I_{k}} e_{j}(f) .
$$

On the sphere $S^{2}$ with the standard metric we have $\Pi=2 \pi, \kappa=2$, and the eigenvalues of the Laplace-Beltrami operator are $\sigma_{k}^{2}-1 / 4=k(k+1), k \geqslant 0$. In this case the operator $E_{k}$ is just the projection onto the space of spherical harmonics of degree $k$. In the general case (assuming periodic geodesic flow) the square roots of the eigenvalues are concentrated near the centers of the intervals $I_{k}$; namely, if $\lambda_{j}$ is large, there is $c \geqslant 0$ independent of $\lambda_{j}$ such that $\sqrt{\lambda_{j}} \subset I_{k}^{*}=\left[\sigma_{k}-c / k, \sigma_{k}+c / k\right]$ for some $k$.

We consider Riesz means of index $\delta$, defined by

$$
S_{R}^{\delta} f(x)=\sum_{k \leqslant R}\left(1-\frac{k}{R}\right)^{\delta} E_{k} f(x) .
$$

We shall show that for $f \in L^{p}, 2<p \leqslant 4$, the Riesz means of positive index converge in norm to $f$. Previously, with the exception of $\mathbb{R}^{2}$ and $S^{2}$ with the standard metric, the sharp $L^{p}$ estimates for Riesz means on two-dimensional manifolds were only known for exponents $p \geqslant 6$ (and, of course, $p=2$ ). These were proved in [31], and by using duality and interpolating with the sharp $L^{\infty}$ estimates in that paper and the trivial $L^{2}$ estimates, one sees that the $L^{4}$ estimate yields sharp results for the summation operators on all $L^{p}$ spaces under the above hypotheses.

We shall actually prove a stronger summability result, namely, that

$$
\left\|\sup _{R>\lambda}\left|S_{R}^{\delta} f-f\right|\right\|_{4} \rightarrow 0 \quad \text { as } \lambda \rightarrow \infty .
$$

This is an immediate consequence of the $L^{4}$ boundedness of the maximal operator $S_{*}^{\delta} f(x)=\sup _{R>0}\left|S_{R}^{\delta} f(x)\right|$, which we shall show for $\delta>0$. This result is new even for spherical harmonics expansions on the sphere $S^{2}$ and generalizes Carbery's maximal theorem [7] for Riesz means in $\mathbb{R}^{2}$. Our maximal estimates follow from sharp $L^{4} \rightarrow L^{4}\left(L^{2}\right)$ local smoothing estimates for the wave operator $\mathscr{F}_{t}=e^{i t \sqrt{-\Delta}}$ and related Fourier integral operators. These imply also new results for more general multiplier operators (i.e., functions of the Laplacian), extending results in $[8,26]$. Moreover, they can be used to prove maximal theorems for a class of oscillatory integrals satisfying a variant of the Carleson-Sjölin condition.

The paper is organized as follows. In the next section we shall give a coordinate free definition of the curvature condition and show how it is related to the Carleson-Sjölin condition. In $\S 3$ we shall prove sharp $L^{2} \rightarrow L^{p}$ local smoothing estimates for Fourier integral operators and inequalities for related square functions. Section 4 will be devoted to $L^{p} \rightarrow L^{p}$ smoothing estimates in higher dimension and in $\S 5$ we consider the related problem of proving variants of the Kakeya maximal theorem. In $\S 6$ we shall prove $L^{p} \rightarrow L^{p}$ smoothing in two dimensions as well as sharp $L^{p} \rightarrow L^{q}\left(L^{2}\right)$ estimates. The latter will be applied in 
$\S 7$ to prove the above-mentioned results concerning multiplier transformations and maximal oscillatory integral operators.

\section{Curvature condition}

As in the introduction, $Y$ and $Z$ are to be $C^{\infty}$ paracompact manifolds of dimension $n$ and $n+1$, respectively. In this paper we shall consider a class of Fourier integral operators $I^{\mu}(Z, Y ; \mathscr{C})$, which is determined by the properties of the canonical relation $\mathscr{C}$.

As usual, we assume that $\mathscr{C}$ is a canonical relation from $T^{*} Y \backslash 0$ to $T^{*} Z \backslash 0$ which is homogeneous, Lagrangian with respect to the symplectic form $d \zeta \wedge d z-d \eta \wedge d y$, and closed in $T^{*} Z \backslash 0 \times T^{*} Y \backslash 0$. Notice that our assumptions imply that $\mathscr{C} \subset T^{*} Z \backslash 0 \times T^{*} Y \backslash 0$ is a conic submanifold of dimension $2 n+1$.

To guarantee nontrivial local regularity properties of operators $\mathscr{F} \in$ $I^{\mu}(Z, Y ; \mathscr{C})$, we shall impose conditions on $\mathscr{C}$ which are based on the properties of the following three projections

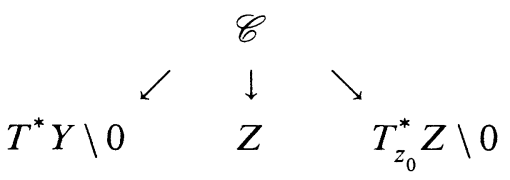

The condition has two parts: (1) a natural nondegeneracy condition which involves the first two projections, and (2) a condition involving the principal curvatures of the images of the projection of $\mathscr{C}$ onto the fibers of $T^{*} Z \backslash 0$.

To describe the first condition, let $\Pi_{T^{*} Y}$ and $\Pi_{Z}$ denote the first two projections in (2.1). We require that they both be submersions, i.e.,

$$
\begin{aligned}
\operatorname{rank} d \Pi_{T^{*} Y} & \equiv 2 n, \\
& \text { rank } d \Pi_{Z} \equiv n+1 .
\end{aligned}
$$

Together, these make up the nondegeneracy requirement. As a side remark, let us point out that if $Y$ and $Z$ were of the same dimension, then (2.2) would imply that $\mathscr{C}$ is locally a canonical graph. Also, in this case the differential in (2.3) would automatically be surjective; however, since we are assuming that the dimensions are different, (2.2) does not imply that $\Pi_{Z}$ is a submersion.

In order to describe the curvature condition, let $z_{0} \in \Pi_{Z} \mathscr{C}$ and let $\Pi_{T_{z_{0}}^{*} Z}$ be the projection of $\mathscr{C}$ onto the fiber $T_{z_{0}}^{*} Z \backslash 0$. Then, clearly,

$$
\Gamma_{z_{0}}=\Pi_{T_{z_{0}}^{*} Z}(\mathscr{C})
$$

is always a conic subset of $T_{z_{0}}^{*} Z \backslash 0$. In fact, $\Gamma_{z_{0}}$ is a smooth immersed hypersurface in $T_{z_{0}}^{*} Z \backslash 0$. In order to see this, note that the first assumption, (2.2), implies that the differential of the projection of $\mathscr{C}$ onto the whole space $T^{*} Z \backslash 0$ must have constant rank $2 n+1$ (see [19, Proposition 4.1.4] and formulae (2.7) and (2.8)). Furthermore, since the differential of $\mathscr{C} \rightarrow T^{*} Z \backslash 0$ 
splits into the differential in the $Z$ direction and in the fiber direction, we see that in view of $(2.3)$

$$
\text { rank } d \Pi_{T_{z_{0}}^{*} Z} \equiv n,
$$

and therefore, by the constant rank theorem, $\Gamma_{z_{0}}$ is a smooth conic $n$-dimensional hypersurface.

Now in addition to the nondegeneracy assumption we shall impose the following

Cone condition: For every $\zeta \in \Gamma_{z_{0}}, n-1$ principal curvatures do not vanish.

Since $\Gamma_{z_{0}}$ is conic, this is the maximum number of curvatures which can be nonzero. Clearly (2.6) does not depend on the choice of local coordinates in $Z$ since changes of variables in $Z$ induce changes of variables in the cotangent bundle which are linear in the fibers.

Let us now see how our assumptions can be reformulated in two useful ways if we use local coordinates. In particular, we shall see that our curvature condition, consisting of (2.2), (2.3), and (2.6) is equivalent to the cinematic curvature condition, defined in [32].

First, we note that (2.2) and (2.3) imply that near a given point $\left(z_{0}, \zeta_{0}, y_{0}, \eta_{0}\right)$ $\in \mathscr{C}$, local coordinates can be chosen so that $\mathscr{C} \ni(z, \zeta, y, \eta) \rightarrow(z, \zeta)$ has bijective differential and, hence, $\mathscr{C}$ can be written here in terms of a generating function. (See [19, Theorem 3.1.3].) Specifically, in these local coordinates, there must be a phase function $\varphi(z, \eta)$ so that $\mathscr{C}$ takes the form

$$
\left\{\left(z, \varphi_{z}^{\prime}(z, \eta), \varphi_{\eta}^{\prime}(z, \eta), \eta\right): \eta \in \mathbb{R}^{n} \backslash 0 \text { in a conic neighborhood of } \eta_{0}\right\} \text {. }
$$

In this case, condition (2.2) becomes

$$
\operatorname{rank} \varphi_{z \eta}^{\prime \prime} \equiv n
$$

which of course means that if we fix $z_{0}$, then, as before,

$$
\Gamma_{z_{0}}=\left\{\varphi_{z}^{\prime}\left(z_{0}, \eta\right): \eta \in \mathbb{R}^{n} \backslash 0 \text { in a conic neighborhood of } \eta_{0}\right\} \subset T_{z_{0}}^{*} Z \backslash 0
$$

must be a smooth conic submanifold of dimension $n$. Then if

$$
\Gamma_{z_{0}} \ni \zeta=\varphi_{z}^{\prime}\left(z_{0}, \eta\right)
$$

and $\theta \in S^{n}$ is normal to $\Gamma_{z_{0}}$ at $\zeta$, it follows that $\pm \theta$ are the unique directions for which

$$
\nabla_{\eta}\left\langle\varphi_{z}^{\prime}\left(z_{0}, \eta\right), \theta\right\rangle=0
$$

The condition that $n-1$ principal curvatures be nonzero at $\zeta$ then is just that

$$
\text { rank }\left(\frac{\partial^{2}}{\partial \eta_{j} \partial \eta_{k}}\right)\left\langle\varphi_{z}^{\prime}\left(z_{0}, \eta\right), \theta\right\rangle=n-1 \text { if } \eta, \theta \text { are as in (2.9). }
$$

It is also convenient to give a formulation which is in the spirit of the wave equation. This involves a splitting of the $z$ variables into "timelike" and "spacelike" directions. If, as above, we work locally, then (2.8) guarantees that we 
can choose coordinates $z=(x, t) \in \mathbb{R}^{n} \times \mathbb{R}$ vanishing at a given point $z_{0}$ so that, first of all,

$$
\operatorname{rank} \varphi_{x \eta}^{\prime \prime} \equiv n
$$

and secondly

$$
\varphi_{t}^{\prime}(x, t, \eta) \neq 0 \text { if } \eta \neq 0 .
$$

In other words, $\Gamma_{z_{0}}$ must be of the form $\Gamma_{z_{0}}=\left\{\left(\varphi_{x}^{\prime}(0, \eta), q\left(\varphi_{x}^{\prime}(0, \eta)\right)\right)\right\}$ for some $q$ satisfying $q(\xi) \neq 0$ if $\xi \neq 0$. This is because, if $(\xi, \tau)$ are the dual variables to $(x, t)$, then $\Gamma_{z_{0}}$ does not intersect the $\tau$ axis. The cone condition, (2.6), just translates here to the condition that rank $q_{\xi \xi}^{\prime \prime} \equiv n-1$. Since $\Gamma_{x, t}$ must have the same form for small $(x, t)$, we see that in appropriate local coordinates

$$
\Gamma_{x, t}=\left\{\left(\varphi_{x}^{\prime}(x, t, \eta), \varphi_{t}^{\prime}(x, t, \eta)\right)\right\}
$$

where $\varphi$ satisfies

$$
\varphi_{t}^{\prime}(x, t, \eta)=q\left(x, t, \varphi_{x}^{\prime}(x, t, \eta)\right) .
$$

Hence local coordinates can always be chosen so that $\mathscr{C}$ is of the form

$$
\mathscr{C}=\left\{(x, t, \xi, \tau, y, \eta):(x, \xi)=\chi_{t}(y, \eta), \tau=q(x, t, \xi) \neq 0\right\},
$$

where, by (2.11), $\chi_{t}$ is a canonical transformation and

$$
\text { rank } q_{\xi \xi}^{\prime \prime} \equiv n-1 \text {. }
$$

This was the formulation given in [32].

Conditions related to (2.14) have already been used in [6], in order to prove $L^{p} \rightarrow L^{p^{\prime}}$ regularity of small time solutions to hyperbolic equations. Closely related is the problem of proving $L^{2} \rightarrow L^{p^{\prime}}$ estimates, which can be reduced to an $L^{p} \rightarrow L^{p^{\prime}}$ estimate for the composition of the Fourier integral operator with its adjoint (see $\S 3)$. Therefore it will be necessary to parameterize the relation $\mathscr{C} \circ \mathscr{C}^{*} \subset T^{*} Z \backslash 0 \times T^{*} Z \backslash 0$ near the diagonal. Here we are using the usual notation that

$$
\mathscr{C}^{*}=\{(y, \eta, z, \zeta):(z, \zeta, y, \eta) \in \mathscr{C}\} .
$$

We assume that local coordinates in $Z$ are chosen such that $\mathscr{C}$ is as in (2.10) and that $\eta$ belongs to the conic set of $\mathbb{R}^{n} \backslash 0$ on which $q$ is defined. Then $\mathscr{C} \circ \mathscr{C}^{*}$ is of the form

$$
\begin{aligned}
\left\{\left(x, t, \eta, \tau, x^{\prime}, t^{\prime}, \xi, \sigma\right):(x, \eta)\right. & =\chi_{t} \circ \chi_{t^{\prime}}^{-1}\left(x^{\prime}, \xi\right), \\
\tau & \left.=q(x, t, \eta), \sigma=q\left(x^{\prime}, t^{\prime}, \xi\right)\right\} .
\end{aligned}
$$

So, if we fix $t^{\prime}$ and let

$$
\mathscr{C}\left(t^{\prime}\right)=\left\{\left(x, t, \eta, \tau, x^{\prime}, \xi\right):(x, \eta)=\chi_{t} \circ \chi_{t^{\prime}}^{-1}\left(x^{\prime}, \xi\right), \tau=q(x, t, \xi)\right\},
$$

we see that $\mathscr{C}\left(t^{\prime}\right)$ is locally parameterized by a phase function of the form $\left\langle x^{\prime}, \eta\right\rangle-\psi\left(x, t, t^{\prime}, \eta\right)$. When $t=t^{\prime}, \chi_{t} \circ \chi_{t^{\prime}}^{-1}$ is the identity, so $\left(x, x^{\prime}, \eta\right) \rightarrow$ $\left\langle x^{\prime}, \eta\right\rangle-\psi\left(x, t^{\prime}, t^{\prime}, \eta\right)$ must parameterize the trivial Lagrangian; that is, 
$\psi\left(x, t^{\prime}, t^{\prime}, \eta\right)=\langle x, \eta\rangle$. This, and the condition that $\tau=q(x, t, \xi)$ in $\mathscr{C}\left(t^{\prime}\right)$, mean that $\psi_{t}^{\prime}\left(x, t^{\prime}, t^{\prime}, \eta\right)=q\left(x, t^{\prime}, \eta\right)$. In other words

$$
\psi\left(x, t, t^{\prime}, \eta\right)=\langle x, \eta\rangle+\left(t-t^{\prime}\right) q\left(x, t^{\prime}, \eta\right)+\left(t-t^{\prime}\right)^{2} r\left(x, t, t^{\prime}, \eta\right)
$$

where $r$ is homogeneous of degree 1 .

Comparisons with the Carleson-Sjölin condition. Let us conclude this section by showing why our curvature condition is the natural homogeneous version of the Carleson-Sjölin condition. The latter is a condition on phase functions $\Phi(z, y)$ so that operators

$$
T_{\lambda} f(z)=\int e^{i \lambda \Phi(z, y)} a(z, y) f(y) d y
$$

satisfy favorable $L^{p}\left(\mathbb{R}^{n}\right) \rightarrow L^{q}\left(\mathbb{R}^{n+1}\right)$ estimates as $\lambda \rightarrow \infty$ (see $\S 3$ ). Here $a \in C_{0}^{\infty}\left(\mathbb{R}^{n+1} \times \mathbb{R}^{n}\right)$.

In Carleson and Sjölin [9], Hörmander [21], and Stein [36] the condition on $\Phi$ has two parts. First there is the following nondegeneracy condition which is the analogue of $(2.8)$ :

$$
\operatorname{rank} \Phi_{z y}^{\prime \prime} \equiv n
$$

If this assumption is met, there is a $\theta \in S^{n}$ so that $\pm \theta$ are the unique directions for which $\nabla_{y}\left\langle\Phi_{z}^{\prime}, \theta\right\rangle=0$. The second part of the Carleson-Sjölin condition then is the following nonhomogeneous analogue of $(2.10)$ :

$$
\operatorname{det}\left(\frac{\partial^{2}}{\partial y_{j} \partial y_{k}}\left\langle\Phi_{z}^{\prime}, \theta\right\rangle\right) \neq 0
$$

One can state these conditions in a coordinate-free way that is similar to the first formulation of our curvature condition. To do this, let

$$
\mathscr{C}_{\Phi}=\left\{\left(z, \Phi_{z}^{\prime}, y,-\Phi_{y}^{\prime}\right)\right\}
$$

be the canonical relation associated to $\Phi$. Unlike before, this submanifold is not homogeneous, but it still is Lagrangian with respect to the symplectic form $d \zeta \wedge d z-d \eta \wedge d y$ in $T^{*} Z \times T^{*} Y$. Now, if $\Pi_{T^{*} Y}$ is the projection from $\mathscr{C}_{\Phi}$ to $T^{*} Y$, then (2.17) is equivalent to the condition that rank $d \Pi_{T^{*} Y} \equiv 2 n$, which is just (2.2). (In the present setting (2.3) is superfluous.) Finally, if $z_{0}$ is in the image of the projection of $\mathscr{C}_{\Phi}$ onto $Z$, and if we let

$$
S_{z_{0}}=\Pi_{T_{z_{0}}^{*} Z}\left(\mathscr{C}_{\Phi}\right)
$$

then, as before, one can use the constant rank theorem to see that $S_{z_{0}}$ is a smooth hypersurface. (2.18) is equivalent to the

$$
\begin{aligned}
& \text { Curvature condition: At every } \zeta \in S_{z_{0}} \text {, the Gaussian curvature } \\
& \text { is nonzero. }
\end{aligned}
$$

This is the analogue of (2.6). Unlike before, it is possible to have $n$ nonvanishing principal curvatures since $S_{z_{0}}$ is not homogeneous; however, for applications (in particular, if we do have homogeneous phase functions) it is useful 
to weaken this assumption. Then we shall just require that for some $k<n$ at least $k$ principal curvatures of $S_{z_{0}}$ do not vanish. Then the rank of the mixed Hessian in (2.18) is at least $k$. In the homogeneous case one recovers the cone condition (2.6) if $k=n-1$.

\section{3. $L^{2} \rightarrow L^{q}$ ESTIMATES}

The main purpose of this section is to prove $L^{2}(Y) \rightarrow L^{q}(Z)$ regularity properties of Fourier integral operators $\mathscr{F}$ of class $I^{\mu-1 / 4}(Z, Y ; \mathscr{C})$ associated to a homogeneous canonical relation $\mathscr{C} \subset T^{*} Z \backslash 0 \times T^{*} Y \backslash 0$, where $Y$ and $Z$ are manifolds of dimension $n$ and $n+1$, respectively. We shall always assume that the projections $d \Pi_{T^{*} Y}, d \Pi_{Z}$ have maximal rank, that is, that $(2.2),(2.3)$ are satisfied. In most cases, we shall also assume that the cone condition (2.6) holds.

Theorem 3.1. Suppose $\mathscr{F} \in I^{\mu-1 / 4}(Z, Y ; \mathscr{C})$, where $\mathscr{C}$ satisfies the nondegeneracy assumptions (2.2), (2.3) and the cone condition (2.6). Suppose that $2(n+1) /(n-1) \leqslant q<\infty$. Then $\mathscr{F}$ is bounded from $L_{\text {comp }}^{2}(Y)$ to $L_{\mathrm{loc}}^{q}(Z)$ if $\mu \leqslant-n(1 / 2-1 / q)+1 / q$.

The kernel of $\mathscr{F}$ can be written as a finite sum of kernels, which in appropriate local coordinate systems are of the form

$$
K(z, y)=\int e^{i[\varphi(z, \eta)-\langle y, \eta\rangle]} a(z, y, \eta) d \eta .
$$

Our assumptions imply that the phase functions $\varphi$ must always satisfy (2.8) and (2.10). We may assume that the symbol $a$ has small conic support in $\mathbb{R}^{n+1} \times \mathbb{R}^{n} \times \mathbb{R}^{n}$; by this we mean that it vanishes for all $(z, y)$ outside a small compact set and for all $\eta=\left(\eta_{1}, \eta^{\prime}\right)$ outside a narrow cone $\left\{\eta:\left|\eta^{\prime}\right| \leqslant \varepsilon \eta_{1}\right\}$. If $a$ is assumed to be of order $\mu$, i.e., if it belongs to the symbol class $S^{\mu}$, then the order of the operator $\mathscr{F}$ is $\mu-1 / 4$, following Hörmander's convention [19].

As we noted in $\S 2$, in most applications we shall want to single out a time variable. Then we write $Z \subset X \times \mathbb{R}$ and denote by $\mathscr{F}_{t}$ the Fourier integral operator with kernel $K_{t}(x, y)=K(x, t, y)$. Then $\mathscr{F}_{t}$ is of class $I^{\mu}\left(X, Y ; \mathscr{C}_{t}\right)$ if we set

$$
\mathscr{C}_{t}=\{(x, \xi, y, \eta):(x, t, \xi, \tau, y, \eta) \in \mathscr{C} \text { for some } \tau\}
$$

Under the assumption that the associated canonical relation $\mathscr{C}_{t}$ is locally the graph of a canonical transformation (which is equivalent to $(2.11)$ ), it was shown in [19] that $(I-\Delta)^{-\mu / 2} \mathscr{F}_{t}$ is bounded on $L^{2}$. Therefore, using the Sobolev embedding theorem one obtains for $2 \leqslant q<\infty$ the sharp inequality

$$
\left\|\mathscr{F}_{t} f\right\|_{q}^{q} \leqslant C\|f\|_{2}^{q}, \quad \mu \leqslant n(1 / 2-1 / q) .
$$

Theorem 3.1 says that if we average the left-hand side over a compact time interval then there is a gain of regularity of order $1 / q$ as soon as $\frac{2(n+1)}{n-1} \leqslant q<\infty$ and the curvature condition is satisfied; namely, one has

$$
\int_{I}\left\|\mathscr{F}{ }_{t} f\right\|_{q}^{q} d t \leqslant C\|f\|_{2}^{q}, \quad \mu \leqslant n(1 / 2-1 / q)-1 / q .
$$


This result is closely related to restriction theorems for the Fourier transform. In fact, the analogue for solutions of the constant coefficient wave equation in $\mathbb{R}^{n+1}$ is a consequence of Strichartz's global space-time estimates [39].

Remarks. Let $\mathscr{F} \in I^{-1 / 4}(Z, Y ; \mathscr{C})$, where $\mathscr{C}$ is as in Theorem 3.1 and let $2(n+1) /(n-1) \leqslant q \leqslant \infty$. A reader who is familiar with Besov spaces can check that our proof implies that $\mathscr{F}$ is bounded from $B_{\alpha, r, \text { comp }}^{2}(Y)$ to $B_{\alpha-\mu, r, \text { loc }}^{q}(Z)$ if $\mu \leqslant n(1 / 2-1 / q)-1 / q$ (cf. (3.5) and (3.6)). For $2 \leqslant r \leqslant q$ this is a slight improvement of Theorem 3.1. Secondly, the cone condition (2.6) can be relaxed. If one assumes that for every $z \in Z, \Gamma_{z}$ has at least $k$ principal curvatures, then the appropriate version of Theorem 3.1 holds for $q \geqslant$ $2(k+2) / k$. If one merely assumes a finite type condition, namely, that for each $\zeta \in \Gamma_{z}$ there is a line tangent to $\Gamma_{z}$ at $\zeta$, which has at most finite order $\ell$ of contact, then one gets the analogous results for $q \geqslant 2 \ell+2$ (see also [27, 36] for related results).

Our second result is an improvement of Theorem 3.1 involving square functions which is related to results on multiplier transformations in $[8,10,25$, 26].

Theorem 3.2. Suppose $\mathscr{F} \in I^{\mu-1 / 4}(X \times I, Y ; \mathscr{C})$, where $\mathscr{C} \subset T^{*} X \backslash 0 \times T^{*} \mathbb{R} \backslash 0$ $\times T^{*} Y \backslash 0$ satisfies the nondegeneracy assumptions (2.2), (2.3). Let $I$ be a compact interval on the real line and

$$
G(f)=\left(\int_{I}|\mathscr{F} f(\cdot, t)|^{2} d t\right)^{1 / 2}
$$

Then $G$ is bounded from $L_{\text {comp }}^{2}(Y)$ to $B M O_{\text {loc }}(X)$ if $\mu \leqslant-(n-1) / 2$.

Suppose that $\mathscr{C}$ also satisfies the cone condition (2.6). Then if $\frac{2(n+1)}{n-1} \leqslant q<$ $\infty$ and $\mu \leqslant-n(1 / 2-1 / q)+1 / 2$, it follows that $G$ is bounded from $L_{\text {comp }}^{2}(Y)$ to $L_{\text {loc }}^{q}(X)$.

Since the canonical relation takes locally the form (2.13), one can apply the Sobolev embedding theorem with respect to the $t$ variable to see that Theorem 3.2 actually implies Theorem 3.1 .

The main application concerns regularity properties of solutions of the wave equation. Let $(M, g)$ be a compact Riemannian manifold and let $\Delta_{g}$ be the Laplace-Beltrami operator. Then if $u$ is the solution of the Cauchy problem

$$
\begin{gathered}
\left((\partial / \partial t)^{2}-\Delta_{g}\right) u=0, \\
\left.u\right|_{t=0}=f,\left.\quad(\partial / \partial t) u\right|_{t=0}=g
\end{gathered}
$$

it follows that $u=\mathscr{F}_{0} f+\mathscr{F}_{1} g$ where $\mathscr{F}_{0} \in I^{-1 / 4}(M \times \mathbb{R}, M ; \mathscr{C})$ and $\mathscr{F}_{1} \in$ $I^{-5 / 4}(M \times \mathbb{R}, M ; \mathscr{C})$. Here, if $\sum_{j k} g^{j k}(x) \xi_{j} \xi_{k}$ is the cometric, then $\mathscr{C}$ is of the form (2.13), where $\chi_{t}$ is the flowout for time $t$ along the HamiltonJacobi vector field associated to \pm the cometric. The function $q$ in $(2.13)$ is just \pm the square-root of the cometric. Consequently, the cinematic curvature assumption is satisfied, since $\chi_{t}$ is a canonical transformation and since (2.14) is fulfilled, due to the fact that $\left(g^{j k}\right)$ is a positive definite matrix. On account of this, we obtain, using Sobolev's embedding theorem in the time variable: 
Corollary 3.3. Let $u$ be the solution to the Cauchy problem (3.2) and assume $2(n+1) /(n-1) \leqslant q<\infty$. Let $2 \leqslant r<\infty$. Then

$$
\left\|\left(\int_{0}^{T}|u(\cdot, t)|^{r} d t\right)^{1 / r}\right\|_{q} \leqslant C_{T}\left(\|f\|_{L_{\alpha}^{2}}+\|g\|_{L_{\alpha-1}^{2}}\right), \quad \alpha=n(1 / 2-1 / q)-1 / r .
$$

The proof of Theorem 3.1 relies on estimates for oscillatory integral operators $T_{\lambda}$ mapping functions on $\mathbb{R}^{n}$ to functions on $\mathbb{R}^{n+1}$. They are defined by

$$
T_{\lambda} f(z)=\int e^{i \lambda \Phi(z, y)} b(z, y) f(y) d y
$$

where $a \in C_{0}^{\infty}\left(\mathbb{R}^{n+1} \times \mathbb{R}^{n}\right)$. Let $\mathscr{C}_{\Phi}$ be the associated canonical relation as in (2.19).

The following result is a slight generalization of a result due to Carleson and Sjölin [9] and Hörmander [21] for $n=1$ and to Stein [36] in higher dimensions. It follows from Stein's argument; however, for the sake of completeness, we shall include a somewhat different proof which does not use an analytic family of operators but instead relies on an argument using fractional integration in one dimension. For previous arguments of this kind see for instance [38, 22].

Proposition 3.4. Suppose that rank $d \Pi_{T^{*} Y} \equiv 2 n$ and that, for each $z_{0}$ in the image of the projection of $\mathscr{C}_{\Phi}$ to $Z, S_{z_{0}}=\Pi_{T_{z_{0}}^{*} Z}\left(\mathscr{C}_{\Phi}\right)$ is an immersed hypersurface in $T_{z_{0}}^{*} Z$. Further, for $0<k \leqslant n$, suppose that at every $\zeta \in S_{z_{0}}$ at least $k$ principal curvatures do not vanish. Then for $1 \leqslant p \leqslant 2$ we have the bounds

$$
\left\|T_{\lambda} f\right\|_{L^{q}(Z)} \leqslant C \lambda^{-(n+1) / q}\|f\|_{L^{p}(Y)}, \quad \lambda>1,
$$

with $q=p^{\prime}(k+2) / k$. As usual $p^{\prime}=p /(p-1)$ so that $1 / p+1 / p^{\prime}=1$.

Proof. We just have to prove the theorem for $p=2$. For then the general case follows by interpolation with the trivial $L^{1} \rightarrow L^{\infty}$ estimate. We use an argument of Tomas according to which the $L^{2} \rightarrow L^{q}$ estimate for $T_{\lambda}$ is equivalent to an $L^{q^{\prime}} \rightarrow L^{q}$ estimate for $T_{\lambda} T_{\lambda}^{*}$. This just follows from duality and the observation that

$$
\int\left|T_{\lambda}^{*} g\right|^{2} d y=\int T_{\lambda} T_{\lambda}^{*} g \bar{g} d z \leqslant\left\|T_{\lambda}^{*} T_{\lambda} g\right\|_{L^{q}}\|g\|_{L^{q^{\prime}}} .
$$

On account of these reductions, we have to prove that

$$
\left\|T_{\lambda} T_{\lambda}^{*} g\right\|_{L^{a_{0}\left(\mathbb{R}^{n+1}\right)}} \leqslant C \lambda^{-\frac{(n+1) k}{k+2}}\|g\|_{L^{q_{0}^{\prime}\left(\mathbb{R}^{n+1}\right)}}, \quad q_{0}=2(k+2) / k .
$$

Let $H^{\lambda}$ be the kernel of $T_{\lambda} T_{\lambda}^{*}$, namely,

$$
H^{\lambda}\left(z, z^{\prime}\right)=\int e^{i \lambda\left[\Phi\left(z^{\prime}, y\right)-\Phi(z, y)\right]} b\left(z^{\prime}, y\right) \overline{b(z, y)} d y .
$$

Using the method of stationary phase one can show that

$$
\left|H^{\lambda}\left(z, z^{\prime}\right)\right| \leqslant C\left(1+\lambda\left|z-z^{\prime}\right|\right)^{-k / 2},
$$

since, as explained in $\S 2$, the assumptions about the curvatures of the hypersurfaces $S_{z_{0}} \subset T^{*} Z_{z_{0}}$ are equivalent to the condition that the mixed Hessian 
in (2.18) has rank $\geqslant k$ everywhere. The proof of (3.3) uses the method of stationary phase and is done in practically the same way as in [36].

If $z, z^{\prime} \in \mathbb{R}^{n+1}$, we may split $z=\left(z_{1}, u\right), z^{\prime}=\left(z_{1}^{\prime}, u^{\prime}\right)$ and assume that $\operatorname{det} \Phi_{u y}^{\prime \prime} \neq 0$ in $\operatorname{supp} a$. For fixed $z_{1}$, let

$$
T_{z_{1}}^{\lambda} f(u)=\int e^{i \lambda \Phi\left(z_{1}, u, y\right)} b\left(z_{1}, u, y\right) f(y) d y .
$$

Then

$$
T_{\lambda} T_{\lambda}^{*} g\left(z_{1}, u\right)=\int T_{z_{1}}^{\lambda}\left(T_{z_{1}^{\prime}}^{\lambda}\right)^{*}\left[g\left(z_{1}^{\prime}, \cdot\right)\right](u) d z_{1}^{\prime}
$$

and for fixed $z_{1}, z_{1}^{\prime}$ the kernel of $T_{z_{1}}^{\lambda}\left(T_{z_{1}^{\prime}}^{\lambda}\right)^{*}$ is just $H^{\lambda}\left(z_{1}^{\prime}, \cdot, z_{1}, \cdot\right)$. By (3.3) the $L^{1}\left(\mathbb{R}^{n}\right) \rightarrow L^{\infty}\left(\mathbb{R}^{n}\right)$ operator norm of $T_{z_{1}}^{\lambda}\left(T_{z_{1}^{\prime}}^{\lambda}\right)^{*}$ is bounded by $\left(1+\lambda\left|z_{1}-z_{1}^{\prime}\right|\right)^{-k / 2}$. The nondegeneracy assumption implies that $T_{z_{1}}^{\lambda}$ is a bounded operator on $L^{2}\left(\mathbb{R}^{n}\right)$ with norm $O\left(\lambda^{-n / 2}\right)$ (see [21]). Therefore the $L^{2}$ operator norm of $T_{z_{1}}^{\lambda}\left(T_{z_{1}^{\prime}}^{\lambda}\right)^{*}$ is $O\left(\lambda^{-n}\right)$, and so, by applying the M. Riesz interpolation theorem, we obtain

$$
\left\|T_{z_{1}}^{\lambda}\left(T_{z_{1}^{\prime}}^{\lambda}\right)^{*} f\right\|_{q} \leqslant C \lambda^{-2 n / q}\left(1+\lambda\left|z_{1}-z_{1}^{\prime}\right|\right)^{-k(1 / 2-1 / q)}\|f\|_{q^{\prime}} .
$$

Note that $1-\left(1 / q_{0}^{\prime}-1 / q_{0}\right)=k\left(1 / 2-1 / q_{0}\right)$. Therefore, we can use the onedimensional Hardy-Littlewood-Sobolev inequality to obtain

$$
\begin{aligned}
& \left\|T_{\lambda} T_{\lambda}^{*} g\right\|_{L^{q_{0}}\left(\mathbb{R}^{n+1}\right)} \leqslant C\left(\int\left(\int\left\|T_{z_{1}}^{\lambda}\left(T_{z_{1}^{\prime}}^{\lambda}\right)^{*}\left[g\left(z_{1}^{\prime}, \cdot\right)\right]\right\|_{q_{0}} d z_{1}^{\prime}\right)^{q_{0}} d z_{1}\right)^{1 / q_{0}} \\
& \leqslant C \lambda^{-(2 n-k) / q_{0}-k 2}\left(\int \left(\int\left|z_{1}-z_{1}^{\prime}\right|^{-k\left(1 / 2-1 / q_{0}\right)}\right.\right. \\
& \left.\left.\times\left\|\left[g\left(z_{1}^{\prime}, \cdot\right)\right]\right\|_{q_{0}^{\prime}} d z_{1}^{\prime}\right)^{q_{0}} d z_{1}\right)^{1 / q_{0}} \\
& \leqslant C \lambda^{-(2 n-k) / q_{0}-k / 2}\left(\int\left\|\left[g\left(z_{1}^{\prime}, \cdot\right)\right]\right\|_{q_{0}^{\prime}}^{q_{0}^{\prime}} d z_{1}^{\prime}\right)^{1 / q_{0}^{\prime}} \\
& =C \lambda^{-(n+1) k /(k+2)}\|g\|_{q_{0}^{\prime}},
\end{aligned}
$$

which finishes the proof.

Proof of Theorem 3.1. In what follows we assume that $a$ is a symbol of order zero, which has small $Z \times Y$ support. Let $\beta \in C_{0}^{\infty}((1 / 2,2))$ satisfy $\sum_{j=-\infty}^{\infty} \beta\left(2^{-j} u\right)=1$ for $u \neq 0$. Set $a_{\lambda}(z, y, \eta)=\beta(|\eta| / \lambda) a(z, y, \eta)$ and let $\mathscr{F}_{\lambda}$ be the operator

$$
\mathscr{F}_{\lambda} f(z)=\iint e^{i[\varphi(z, \eta)-\langle y, \eta\rangle]} a_{\lambda}(z, y, \eta) f(y) d \eta d y .
$$

Put $q_{0}=2(n+1) /(n-1)$. Then for $q_{0} \leqslant q \leqslant \infty$ we shall prove that

$$
\left\|\mathscr{F}_{\lambda} f\right\|_{L^{q}\left(\mathbb{R}^{n+1}\right)} \leqslant C \lambda^{n(1 / 2-1 / q)-1 / q}\|f\|_{L^{2}\left(\mathbb{R}^{n}\right)}, \quad \lambda>1
$$


Let us first see that (3.4) implies the statement in the theorem. For sufficiently large fixed $c_{0}$, we fix a $\widetilde{\beta} \in C_{0}^{\infty}\left(\left(c_{0}^{-1} / 2,2 c_{0}\right)\right)$, which equals one in $\left(c_{0}^{-1}, c_{0}\right)$. We shall use Littlewood-Paley operators $L_{j}$ which for $j>0$ are defined by setting $\widehat{L_{j} f}=\widetilde{\beta}\left(2^{-j}|\eta|\right) \widehat{f}(\eta)$, where $\hat{f}$ denotes the Fourier transform in $\mathbb{R}^{n}$. Analogously, we define Littlewood-Paley operators in $\mathbb{R}^{n+1}, L_{j}^{\prime} g$, in such a way that the Fourier transform of $L_{j}^{\prime} g$ is supported in a dyadic annulus in $\mathbb{R}^{n+1}$ of width $\approx c_{0} 2^{j}$. Finally $L_{0} f$ and $L_{0}^{\prime} g$ are defined in the obvious way to capture the low frequencies.

We can use a straightforward integration by parts to see that the $L^{2}\left(\mathbb{R}^{n}\right) \rightarrow$ $L^{2}\left(\mathbb{R}^{n+1}\right)$ operator norms of $\left(I-L_{j}^{\prime}\right) \mathscr{F}_{2^{j}}$ and $\mathscr{F}_{2^{j}}\left(I-L_{j}\right)$ are $O\left(2^{-j N}\right)$ for any $N>0$, provided $c_{0}$ in the definition of $\tilde{\beta}$ is chosen large enough. These arguments are used in the proof of the theorem on compositions of Fourier integral operators (see [19, p.177]). They rely on the homogeneity of the phase function and the assumption that $\mathscr{C}$ has empty intersection with both $T^{*} Z \times 0$ and $0 \times T^{*} Y$. One may apply the Sobolev embedding theorem to also see that

$$
\left\|\left(I-L_{j}^{\prime}\right) \mathscr{F}_{2^{j}} f\right\|_{q}+\left\|\mathscr{F}_{2^{j}}\left(I-L_{j}\right) f\right\|_{q} \leqslant C_{N} 2^{-j N}\|f\|_{2} .
$$

Furthermore, if $C_{q}$ denotes the constant in (3.4) and if $\gamma_{q}=n(1 / 2-1 / q)-1 / q$, then we obtain

$$
\left(\sum_{j \geqslant 0} 2^{-2 j \gamma_{q}}\left\|L_{j^{\prime}}^{\prime} \mathscr{F}_{2^{j}} L_{j} f\right\|_{q}^{2}\right)^{1 / 2} \leqslant C_{q}\left(\sum_{j \geqslant 0}\left\|L_{j} f\right\|_{2}^{2}\right)^{1 / 2} \leqslant C_{q}^{\prime}\|f\|_{2} .
$$

Since, by Littlewood-Paley theory,

$$
\begin{aligned}
& \left\|\sum_{j \geqslant 0} 2^{-j \gamma_{q}} L_{j}^{\prime} \mathscr{F}_{2^{j}} L_{j} f\right\|_{q} \leqslant c_{q}\left\|\left(\sum_{j \geqslant 0}\left|2^{-j \gamma_{q}} L_{j}^{\prime} \mathscr{F}_{2^{j}} L_{j} f\right|^{2}\right)^{1 / 2}\right\|_{q} \\
& \leqslant c_{q}\left(\sum_{j \geqslant 0} 2^{-2 j \gamma_{q}}\left\|L_{j}^{\prime} \mathscr{F}_{2^{j}} L_{j} f\right\|_{q}^{2}\right)^{1 / 2}
\end{aligned}
$$

we see that Theorem 3.1 follows from (3.5) and (3.6). It remains to prove (3.4). We use the calculus of Fourier integrals to see that

$$
\begin{aligned}
\mathscr{F}_{\lambda} f(z) & =\int e^{i \varphi(z, \eta)} \tilde{a}_{\lambda}(z, \eta) \widehat{f}(\eta) d \eta+\mathscr{R}_{\lambda} f(z) \\
& =\lambda^{n} \int e^{i \lambda \varphi(z, y)} \tilde{a}_{\lambda}(z, \lambda y) \widehat{f}(\lambda y) d y+\mathscr{R}_{\lambda} f(z) .
\end{aligned}
$$

Here $\tilde{a}_{\lambda}$ is supported in a dyadic annulus of width $\approx \lambda$ and $\mathscr{R}_{\lambda} \in I^{-5 / 4}(Z, Y ; \mathscr{C})$ is a remainder term such that

$$
\left\|\mathscr{R}_{\lambda} f\right\|_{q} \leqslant C \lambda^{n(1 / 2-1 / q)-1}\|f\|_{2} .
$$

Note that $(z, y) \rightarrow \tilde{a}_{\lambda}(z, \lambda y)$ is a $C^{\infty}$ function with bounds independent of $\lambda$ which is supported in a compact set independent of $\lambda$. Therefore (3.4) 
for $q=\infty$ is trivial. We may apply Proposition 3.4 to the main term in (3.7) to obtain

$$
\begin{aligned}
\left\|\mathscr{F}_{\lambda} f\right\|_{q_{0}} & \leqslant C \lambda^{n} \lambda^{-(n+1) / q_{0}}\|\widehat{f}(\lambda \cdot)\|_{2}+C \lambda^{n(1 / 2-1 / q)-1}\|f\|_{2} \\
& \leqslant C \lambda^{n\left(1 / 2-1 / q_{0}\right)-1 / q_{0}}\|f\|_{2} .
\end{aligned}
$$

This together with another interpolation finishes the proof.

Proof of Theorem 3.2. For $\mathscr{F}_{\lambda}$ as above we shall prove the inequality

$$
\left\|\mathscr{F}_{\lambda} f\right\|_{L^{q}\left(\mathbb{R}^{n}, L^{2}(\mathbb{R})\right)} \leqslant C \lambda^{n(1 / 2-1 / q)-1 / 2}\|f\|_{L^{2}\left(\mathbb{R}^{n}\right)}
$$

for $2(n+1) /(n-1)=q_{0} \leqslant q \leqslant \infty$. Again, by Tomas' argument, this follows from

$$
\left\|\mathscr{F}_{\lambda}\left(\mathscr{F}_{\lambda}\right)^{*} f\right\|_{L^{q}\left(\mathbb{R}^{n}, L^{2}(\mathbb{R})\right)} \leqslant C \lambda^{2 n(1 / 2-1 / q)-1}\|f\|_{L^{q^{\prime}}\left(\mathbb{R}^{n}\right)^{\circ}}
$$

We may write the kernel $K^{\lambda}$ of $\mathscr{F}_{\lambda}\left(\mathscr{F}_{\lambda}\right)^{*}$ as

$$
K^{\lambda}\left(x, t, x^{\prime}, t^{\prime}\right)=\int e^{i\left[\psi\left(x, t, t^{\prime}, \eta\right)-\left\langle x^{\prime}, \eta\right\rangle\right]} b_{\lambda}\left(x, t, x^{\prime}, t^{\prime}, \eta\right) d \eta,
$$

where $\psi$ satisfies (2.16). Assuming small conic support of $a_{\lambda}$ in the definition of $\mathscr{F}_{\lambda}$ we see that $b$ vanishes if $(x, t)$ is not close to $\left(x^{\prime}, t^{\prime}\right)$. Moreover, the $\eta$ support of $b_{\lambda}$ is contained in a dyadic annulus of width $\approx \lambda$, and we may assume that $b_{\lambda}$ vanishes for all $\eta$ outside a narrow cone containing the $\eta_{1}$ axis. We split $x=\left(x_{1}, v\right), x^{\prime}=\left(x_{1}^{\prime}, v^{\prime}\right)$. Then, for fixed $x_{1}, x_{1}^{\prime}$, we define the operators $T_{x_{1} x_{1}^{\prime}}^{\lambda}$ by

$$
T_{x_{1} x_{1}^{\prime}}^{\lambda} h(v, t)=\iint K^{\lambda}\left(x_{1}, v, t, x_{1}^{\prime}, v^{\prime}, t^{\prime}\right) h\left(v^{\prime}, t^{\prime}\right) d v^{\prime} d t^{\prime}
$$

We shall establish the following inequalities:

$$
\begin{aligned}
& \left\|\left(\int\left|T_{x_{1} x_{1}^{\prime}}^{\lambda} h(\cdot, t)\right|^{2} d t\right)^{1 / 2}\right\|_{L^{2}\left(\mathbb{R}^{n-1}\right)} \leqslant C\left\|\left(\int\left|h\left(\cdot, t^{\prime}\right)\right|^{2} d t^{\prime}\right)^{1 / 2}\right\|_{L^{2}\left(\mathbb{R}^{n-1}\right)}, \\
& \left\|\left(\int\left|T_{x_{1} x_{1}^{\prime}}^{\lambda} h(\cdot, t)\right|^{2} d t\right)^{1 / 2}\right\|_{L^{\infty}\left(\mathbb{R}^{n-1}\right)} \\
& \leqslant C \lambda^{n-1}\left(1+\lambda\left|x_{1}-x_{1}^{\prime}\right|\right)^{-(n-1) / 2}\left\|\left(\int\left|h\left(\cdot, t^{\prime}\right)\right|^{2} d t^{\prime}\right)^{1 / 2}\right\|_{L^{1}\left(\mathbb{R}^{n-1}\right)}
\end{aligned}
$$

An analogue of (3.10) without the decay factor $\left(1+\lambda\left|x_{1}-x_{1}^{\prime}\right|\right)^{-(n-1) / 2}$ remains valid if we do not assume the cone condition.

Assuming (3.9) and (3.10) for a moment we may proceed as in the proof of Proposition 3.4. Observe that

$$
\mathscr{F}_{\lambda} \mathscr{F}_{\lambda}^{*} g\left(x_{1}, v, t\right)=\int T_{x_{1} x_{1}^{\prime}}^{\lambda}\left[g\left(x_{1}^{\prime}, \cdot\right)\right](v, t) d x_{1}^{\prime},
$$


and use Sobolev embedding to obtain

$$
\begin{aligned}
& \left\|\mathscr{F}_{\lambda} \mathscr{F}_{\lambda}^{*} g\right\|_{L^{q_{0}\left(\mathbb{R}^{n}, L^{2}(\mathbb{R})\right)}} \\
& \leqslant C\left(\int\left(\int\left\|T_{x_{1} x_{1}^{\prime}}^{\lambda}\left[g\left(x_{1}^{\prime}, \cdot\right)\right]\right\|_{L^{q_{0}}\left(\mathbb{R}^{n-1}, L^{2}(\mathbb{R})\right)} d x_{1}^{\prime}\right)^{q_{0}} d x_{1}\right)^{1 / q_{0}} \\
& \leqslant C \lambda^{-1+2 / q_{0}}\left(\int \left(\int \lambda^{n-2 n / q_{0}}\left(1+\lambda\left|x_{1}-x_{1}^{\prime}\right|\right)^{-(n-1)\left(1 / 2-1 / q_{0}\right)}\right.\right. \\
& \left.\left.\times\left\|g\left(x_{1}^{\prime}, \cdot\right)\right\|_{L^{q_{0}\left(\mathbb{R}^{n-1}, L^{2}(\mathbb{R})\right)}} d x_{1}^{\prime}\right)^{q_{0}} d x_{1}\right)^{1 / q_{0}} \\
& \leqslant C \lambda^{(n-1) /(n+1)}\left(\int\left\|g\left(x_{1}^{\prime}, \cdot\right)\right\|_{L^{q_{0}^{\prime}\left(\mathbb{R}^{n-1}, L^{2}(\mathbb{R})\right)}}^{q^{\prime}} d x_{1}^{\prime}\right)^{1 / q_{0}^{\prime}} \\
& =C \lambda^{n-2 n / q_{0}-1}\|g\|_{L^{q_{0}^{\prime}}\left(\mathbb{R}^{n}, L^{2}(\mathbb{R})\right)} \text {. }
\end{aligned}
$$

This proves (3.8) for $q=q_{0}$, while for $q=\infty$ (3.8) follows from the proof of (3.10) (here the cone condition is not necessary). Finally one can use a Littlewood-Paley decomposition $\left\{L_{j}\right\}$ in $\mathbb{R}^{n}$ and use similar arguments as in the proof of Theorem 3.1 to see that, for $q_{0} \leqslant q \leqslant \infty$,

$$
\left(\sum_{j=0}^{\infty}\left\|\left(\int\left|L_{j} \mathscr{F} f(\cdot, t)\right|^{2} d t\right)^{1 / 2}\right\|_{q}^{2}\right)^{1 / 2} \leqslant C\|f\|_{2}
$$

which is somewhat stronger than the asserted $L^{2} \rightarrow L^{q}$ and $L^{2} \rightarrow B M O$ inequalities for the square function.

Proof of (3.9). For fixed $x_{1}, x_{1}^{\prime}$ we have to prove the boundedness of the Fourier integral operator $T_{x_{1}^{\prime} x_{1}^{\prime}}^{\lambda}$ on $L^{2}\left(\mathbb{R}^{n}\right)$. It is defined by means of a phase function $\Psi$, depending smoothly on the parameters $x_{1}, x_{1}^{\prime}$. If we split $\eta=$ $\left(\eta_{1}, \zeta\right)$, then $\Psi$ is given by

$$
\Psi\left(v, t, v^{\prime}, t^{\prime}, \eta\right)=\psi\left(x_{1}, v, t, t^{\prime}, \eta\right)-x_{1}^{\prime} \eta_{1}-\left\langle v^{\prime}, \zeta\right\rangle
$$

We have to show that the associated canonical relation is locally the graph of a canonical transformation. According to [19, Proposition 4.1.3] this follows if

$$
\operatorname{det}\left(\begin{array}{cc}
\Psi_{(v, t)\left(v^{\prime}, t^{\prime}\right)}^{\prime \prime} & \Psi_{(v, t) \eta}^{\prime \prime} \\
\Psi_{\eta\left(v^{\prime}, t^{\prime}\right)}^{\prime \prime} & \Psi_{\eta \eta}^{\prime \prime}
\end{array}\right) \neq 0
$$

Observe that since $\Psi_{(v, t)\left(v^{\prime}, t^{\prime}\right)}^{\prime \prime}=0,(3.11)$ follows from $\operatorname{det} \Psi_{(v, t) \eta}^{\prime \prime} \neq 0$ and $\operatorname{det} \Psi_{\eta\left(v^{\prime}, t^{\prime}\right)}^{\prime \prime} \neq 0$. Using (2.16) we see that

$$
\Psi_{\eta\left(v^{\prime}, t^{\prime}\right)}^{\prime \prime}=\left(\begin{array}{cc}
0 & \psi_{\eta_{1} t^{\prime}}^{\prime \prime} \\
I_{n-1} & \psi_{\zeta t^{\prime}}^{\prime \prime}
\end{array}\right)=\left(\begin{array}{cc}
0 & q_{\eta_{1}}^{\prime} \\
I_{n-1} & q_{\zeta}^{\prime}
\end{array}\right)+O\left(t-t^{\prime}\right)
$$

and

$$
\Psi_{(v, t) \eta}^{\prime \prime}=\left(\begin{array}{ll}
\psi_{v \eta_{1}}^{\prime \prime} & \psi_{v \zeta}^{\prime \prime} \\
\psi_{t \eta_{1}}^{\prime \prime} & \psi_{t \zeta}^{\prime \prime}
\end{array}\right)=\left(\begin{array}{cc}
0 & I_{n-1} \\
q_{\eta_{1}}^{\prime} & q_{\zeta}^{\prime}
\end{array}\right)+O\left(t-t^{\prime}\right) .
$$

Recall that $q \neq 0$. Therefore, since $q$ homogeneous of degree 1 and since we are assuming that the symbol is supported in a narrow cone containing the $\eta_{1}$ 
axis, we have also $q_{\eta_{1}}^{\prime} \neq 0$ in $\operatorname{supp} b_{\lambda}$. Since we are also assuming that $t-t^{\prime}$ is small, we conclude that (3.11) must hold and this implies (3.9).

Proof of (3.10). It follows from (2.14) that, in the support of $b_{\lambda}$, the level sets

$$
S\left(x, t^{\prime}\right)=\left\{\eta: q\left(x, t^{\prime}, \eta\right)=1\right\}
$$

have nonzero Gaussian curvature. We introduce polar coordinates $\eta=\rho \eta(u)$ such that $\eta(u) \in S\left(x, t^{\prime}\right)$ and Lebesgue measure transforms via $d \eta=$ $\rho^{n-1} d \rho g\left(x, t^{\prime}, u\right) d u$ with a smooth density $g$, depending smoothly on $x$ and $t^{\prime}$. Then using (2.16) we see that we can write $K^{\lambda}$ as

$$
\begin{aligned}
K^{\lambda}\left(x, t, x^{\prime}, t^{\prime}\right)=\iint & e^{i \rho\left[\left\langle x-x^{\prime}, \eta(u)\right\rangle+\left(t-t^{\prime}\right)+\left(t-t^{\prime}\right)^{2} r\left(x, t, t^{\prime}, \eta(u)\right)\right]} \\
& \times b_{\lambda}\left(x, t, x^{\prime}, t^{\prime}, \rho \eta(u)\right) g\left(x, t^{\prime}, u\right) d u \rho^{n-1} d \rho .
\end{aligned}
$$

If $t=t^{\prime}$, the phase in (3.12), considered as a function of $u \in \mathbb{R}^{n-1}$, becomes stationary if $x-x^{\prime}$ is normal to $S\left(x, t^{\prime}\right)$ at $\eta(u)$. Since $t-t^{\prime}$ is small we may apply the method of stationary phase and obtain

$$
\begin{aligned}
K^{\lambda}\left(x, t, x^{\prime}, t^{\prime}\right)= & \int e^{i \rho\left[\left(\psi_{0}\left(x, x^{\prime}, t, t^{\prime}\right)+\left(t-t^{\prime}\right)+\left(t-t^{\prime}\right)^{2} \psi_{1}\left(x, x^{\prime}, t, t^{\prime}\right)\right]\right.} \\
& \times\left(\sum_{j=0}^{N} b_{\lambda, j}\left(x, t, x^{\prime}, t^{\prime}, \rho\right)+R_{\lambda, N}\left(x, t, x^{\prime}, t^{\prime}, \rho\right)\right) \rho^{n-1} d \rho,
\end{aligned}
$$

where $\psi_{0}, \psi_{1}$ are smooth with

$$
\psi_{0}\left(x^{\prime}, x^{\prime}, t, t^{\prime}\right)=0
$$

and $b_{\lambda, j}$ and $R_{\lambda, N}$ are symbols of order zero, supported in a dyadic interval where $\rho \approx \lambda$, such that

$$
\begin{aligned}
\partial_{\rho}^{\alpha} b_{\lambda, j}\left(x, t, x^{\prime}, t^{\prime}, \rho\right) & \leqslant C_{\alpha}\left(1+\lambda\left|x-x^{\prime}\right|\right)^{-(n-1) / 2-j}(1+\rho)^{-\alpha}, \\
\partial_{\rho}^{\alpha} R_{\lambda, N}\left(x, t, x^{\prime}, t^{\prime}, \rho\right) & \leqslant C_{\alpha}(1+\rho)^{-\alpha}\left(1+\lambda\left|x-x^{\prime}\right|\right)^{-N} .
\end{aligned}
$$

In (3.13) we may integrate by parts with respect to $\rho$ and then we obtain the bound

$$
\begin{aligned}
\left|K^{\lambda}\left(x, t, x^{\prime}, t^{\prime}\right)\right| & \leqslant C_{N} \lambda^{n}\left(1+\lambda\left|x-x^{\prime}\right|\right)^{-(n-1) / 2} \\
& \times\left(1+\lambda\left|\psi_{0}\left(x, x^{\prime}, t, t^{\prime}\right)+\left(t-t^{\prime}\right)+\left(t-t^{\prime}\right)^{2} \psi_{1}\left(x, x^{\prime}, t, t^{\prime}\right)\right|\right)^{-N} .
\end{aligned}
$$

Using (3.14) and the implicit function theorem, we see that for fixed $x, x^{\prime}, t^{\prime}$ we can solve the equation

$$
\psi_{0}\left(x, v, t, t^{\prime}\right)+\left(t-t^{\prime}\right)+\left(t-t^{\prime}\right)^{2} \psi_{1}\left(x, x^{\prime}, t, t^{\prime}\right)=0
$$

by means of a function $t=\tilde{t}\left(x, x^{\prime}, t^{\prime}\right)$ such that $\tilde{t}(x, x, t)=t$ and $(\partial \tilde{t}) /(\partial t)>0$. We obtain the bound

$$
K^{\lambda}\left(x, t, x^{\prime}, t^{\prime}\right) \leqslant C_{N} \lambda^{n}\left(1+\lambda\left|x-x^{\prime}\right|\right)^{-(n-1) / 2}\left(1+\lambda\left|t-\tilde{t}\left(x, x^{\prime}, t^{\prime}\right)\right|\right)^{-N}
$$


Thus, straightforward applications of the Minkowski and the Schwarz inequality yield

$$
\begin{aligned}
& \lambda^{-n}\left(1+\lambda\left|x-x^{\prime}\right|\right)^{(n-1) / 2}\left(\int\left|T_{x_{1} x_{1}^{\prime}}^{\lambda} h(v, t)\right|^{2} d t\right)^{1 / 2} \\
& \leqslant C\left(\int\left|\iint\left(1+\lambda\left|t-\tilde{t}\left(x, x^{\prime}, t^{\prime}\right)\right|\right)^{-N} h\left(v^{\prime}, t^{\prime}\right) d t^{\prime} d v^{\prime}\right|^{2} d t\right)^{1 / 2} \\
& \leqslant C \int\left(\iint\left(1+\lambda\left|t-\tilde{t}\left(x, x^{\prime}, t^{\prime}\right)\right|\right)^{-N} d t^{\prime}\right. \\
& \left.\quad \times \int\left(1+\lambda\left|t-\tilde{t}\left(x, x^{\prime}, t^{\prime}\right)\right|\right)^{-N}\left|h\left(v^{\prime}, t^{\prime}\right)\right|^{2} d t^{\prime} d t\right)^{1 / 2} d v^{\prime} \\
& \leqslant C \lambda^{-1 / 2} \int\left(\iint\left(1+\lambda\left|t-\tilde{t}\left(x, x^{\prime}, t^{\prime}\right)\right|\right)^{-N} d t\left|h\left(v^{\prime}, t^{\prime}\right)\right|^{2} d s\right)^{1 / 2} d v^{\prime} \\
& \leqslant C \lambda^{-1} \int\left(\int\left|h\left(v^{\prime}, t^{\prime}\right)\right|^{2} d s\right)^{1 / 2} d v^{\prime} .
\end{aligned}
$$

This concludes the proof of Theorem 3.2.

\section{4. $L^{p} \rightarrow L^{p}$ LOCAL SMOOTHING IN HIGHER DIMENSIONS}

In this section we investigate the $L^{p} \rightarrow L^{p}$ local smoothing properties of Fourier integral operators which satisfy the cinematic curvature condition. Since the methods in this section cannot be used to prove local smoothing in dimension two, we postpone the study of such operators until the next section. There a somewhat different approach, relying on geometric arguments, will be used to obtain local smoothing.

We split up the main results of this section since they require different proofs. Unlike those in the previous section, the local smoothing estimates we obtain here are probably not sharp; however, at the end of this section, we shall see that for certain exponents $p>2$ we can prove sharp $L^{p} \rightarrow L^{p}$ local smoothing for Fourier integrals of type $(1 / 2,1 / 2)$.

Theorem 4.1. Let $n \geqslant 4$ and suppose that $\mathscr{F} \in I^{\mu-1 / 4}(Z, Y ; \mathscr{C})$ where, as before, $\mathscr{C}$ satisfies the nondegeneracy assumption $(2.2),(2.3)$ and the cone condition (2.6). Then $\mathscr{F}: L_{\text {comp }}^{p}(Y) \rightarrow L_{\text {loc }}^{p}(Z)$ if $\mu \leqslant-(n-1)(1 / 2-1 / p)+\varepsilon(p, n)$, where

$$
\varepsilon(p, n)= \begin{cases}\frac{n-3}{n-1} \frac{1}{p}, & \frac{2(n+1)}{n-1} \leqslant p<\infty, \\ \frac{n-3}{2}\left(\frac{1}{2}-\frac{1}{p}\right), & 2<p \leqslant \frac{2(n+1)}{n-1} .\end{cases}
$$

Thus, there is local smoothing of order $\varepsilon(p, n)$ (see the introduction). We shall see that this result is actually a corollary of Theorem 3.1. However, the next one is more difficult.

Theorem 4.2. Let $n=3$ and suppose that $\mathscr{F} \in I^{\mu-1 / 4}(Z, Y ; \mathscr{C})$ where $\mathscr{C}$ satisfies the nondegeneracy assumption (2.2), (2.3) and the cone condition (2.6). It then follows that $\mathscr{F}: L_{\text {comp }}^{p}(Y) \rightarrow L_{\mathrm{loc}}^{p}(Z)$ if $\mu<-2(1 / 2-1 / p)+\varepsilon(p)$, 
where

$$
\varepsilon(p)= \begin{cases}\frac{1}{2 p}, & 4 \leqslant p<\infty, \\ \frac{1}{2}\left(\frac{1}{2}-\frac{1}{p}\right), & 2<p \leqslant 4 .\end{cases}
$$

In $\S 6$ we shall see that in two dimensions there is also local smoothing of order $\varepsilon(p)$ given by (4.2).

Before turning to the proofs, let us state a couple of corollaries of the $L^{p}$ local smoothing estimates. If we write $z=(x, t)$ as before and let $\mathscr{F} f(x)=$ $\mathscr{F} f(x, t)$ then we have the following maximal theorem under the above assumptions:

Corollary 4.3. Assume that $\mathscr{F} \in I^{\mu-1 / 4}(Z, Y ; \mathscr{C})$. Then, if $I$ is a compact interval and $X$ and $Y$ are assumed to be compact

$$
\left\|\sup _{t \in I}|\mathscr{F} f(x)|\right\|_{L^{p}(X)} \leqslant C\|f\|_{L^{p}(Y)},
$$

if $\mu<-(n-1)(1 / 2-1 / p)-(1 / p-\varepsilon(p, n))$, where $\varepsilon(p, n)$ is given by (4.1) when $n \geqslant 4$ and by (4.2) when $n=3$.

This and similar results in two dimensions stated in $\S 6$ below improve results in $[4,32,34,35]$. In particular, here we do not need to assume that the operators $\mathscr{F}_{t}$ are conormal. As in [24], one proves (4.3) via an easy argument involving the local smoothing estimates and an application of the Sobolev embedding theorem in the $t$ variable. An interesting problem would be to try to determine the sharp version of (4.3). A conjecture would be that if $p \geqslant 2 n /(n-1)$, then (4.3) holds if $\mu<-(n-1)(1 / 2-1 / p)$. Notice that this would say that, for this range of exponents, the sharp $L^{p}(Y) \rightarrow L^{p}(X)$ regularity properties of the operators $\mathscr{F}_{t}$ and the associated maximal operator are essentially the same.

A related corollary concerns the regularity properties of the solution to the Cauchy problem (3.2). In [28] it was shown that if the initial data $f=$ $\left.u\right|_{t=0}, g=\left.\partial_{t} u\right|_{t=0}$ belong to $L_{\alpha}^{p}(M)$ and $L_{\alpha-1}^{p}(M)$ then $u(\cdot, t) \in L_{\alpha-\beta_{p}}^{p}(M)$, where $\beta_{p}=(n-1)|1 / 2-1 / p|$. Furthermore, this result is sharp for all but a discrete set of $t$. The next result says that, if one instead considers the more natural problem of the local regularity of $u$ as a function of $x$ and $t$, then, for $2<p<\infty$, there is a gain of regularity versus the fixed time estimate.

Corollary 4.4. Let $u$ be the solution to the Cauchy problem (3.2). Then, if $I \subset \mathbb{R}$ is a compact interval and if $\varepsilon<\varepsilon(p, n)$, with $\varepsilon(p, n)$ as above,

$$
\|u\|_{L_{\alpha-\beta_{p}+\varepsilon}^{p}(M \times I)} \leqslant C\left(\|f\|_{L_{\alpha}^{p}(M)}+\|g\|_{L_{\alpha}^{p}(M)}\right), \quad 2<p<\infty .
$$

Proof of Theorem 4.1. Results in [19] and [28] imply that if $\mathscr{F} \in$ $I^{\mu-1 / 4}(Z, Y ; \mathscr{C})$ then $\mathscr{F}$ is bounded from $L_{\text {comp }}^{2}(Y)$ to $L_{\text {loc }}^{2}(Z)$ if $\mu \leqslant 0$ and bounded from $L_{\text {comp }}^{\infty}(Y)$ to $B M O_{\text {loc }}(Z)$ if $\mu \leqslant-(n-1) / 2$. So, by applying analytic interpolation, Theorem 4.1 must follow from the special case

$$
\begin{gathered}
\mathscr{F}: L_{\mathrm{comp}}^{p_{0}}(Y) \rightarrow L_{\mathrm{loc}}^{p_{0}}(Z), \quad p_{0}=\frac{2(n+1)}{n-1}, \\
\mu \leqslant-\frac{n-3}{2(n+1)}=-(n-1)\left(\frac{1}{2}-\frac{1}{p_{0}}\right)+\frac{n-3}{n-1} \frac{1}{p_{0}} .
\end{gathered}
$$


But $\frac{n-3}{2(n+1)}=n\left(1 / 2-1 / p_{0}\right)-1 / p_{0}$, so Theorem 3.1 says that the stronger version of (4.4), where $L_{\text {comp }}^{p_{0}}(Y)$ is replaced by $L_{\text {comp }}^{2}(Y)$, is true. Consequently, (4.4) follows from Theorem 3.1 and Hölder's inequality.

Proof of Theorem 4.2. As above, if one uses interpolation, one sees that the results follow if we can show that $\mathscr{F} \in I^{-1 / 4}(Z, Y ; \mathscr{C})$ is bounded from $L_{\text {comp }}^{4}(Y)$ to $L_{\mu, \text { loc }}^{4}(Z)$ if $\mu<-1 / 2+1 / 8$. We shall use the $L^{2} \rightarrow L^{4}$ smoothing estimates of the previous section to see that the $L^{4}$ norm of $\mathscr{F} f$ is controlled by the $L^{4}$ norm of a certain square function involving a natural decomposition of the operators $\mathscr{F}$. This square function can be estimated if one can prove $L^{2}$ estimates for variable coefficient Kakeya maximal functions. Those estimates will be proved in the subsequent section.

As before, if we work microlocally, we may assume that $\mathscr{F}$ is of the form

$$
\mathscr{F} f(z)=\int_{\mathbb{R}^{3}} e^{i \varphi(z, \eta)} a(z, \eta) \hat{f}(\eta) d \eta,
$$

where the phase function $\varphi$ satisfies the conditions described in $\S 2$, and where $a$ is assumed to be a symbol of order $\mu$ which is compactly supported in $z \in \mathbb{R}^{3}$.

Following the proof of Theorem 3.1, we fix $\beta \in C_{0}^{\infty}(\mathbb{R})$ vanishing for $|s| \notin$ $[1 / 2,2]$ and set $a_{\lambda}(z, \eta)=\lambda^{-\mu} \beta(|\eta| / \lambda) a(z, \eta)$. Then $a_{\lambda}$ is a symbol of order zero and satisfies the usual symbol estimates uniformly in $\lambda$. If we put

$$
\mathscr{F}_{\lambda} f(z)=\int e^{i \varphi(z, \eta)} a_{\lambda}(z, \eta) \hat{f}(\eta) d \eta,
$$

then, by summing a geometric series, we see that it suffices to prove the dyadic estimates

$$
\left\|\mathscr{F}_{\lambda} f\right\|_{L^{4}\left(\mathbb{R}^{4}\right)} \leqslant C_{\varepsilon} \lambda^{3 / 8+\varepsilon}\|f\|_{L^{4}\left(\mathbb{R}^{3}\right)}, \quad \lambda>1, \quad \varepsilon>0 .
$$

To handle (4.6) we need to make a decomposition with respect to the $\eta$ variables. To do this, we first must define homogeneous partitions of unity of $\mathbb{R}^{3} \backslash 0$ that depend on the scale $\lambda$. Specifically, we choose $C^{\infty}$ functions $\chi_{\nu}, \nu=1, \ldots, N(\lambda) \approx \lambda$, satisfying $\sum \chi_{\nu} \equiv 1$ and having the following properties. First, the $\chi_{\nu}$ are to be homogeneous of degree zero and satisfy the estimates

$$
\left|\partial^{\alpha} \chi_{\nu}(\eta)\right| \leqslant C_{\alpha} \lambda^{|\alpha| / 2} \quad \forall \alpha \text { if }|\eta|=1 .
$$

Furthermore, if unit vectors $\eta_{\nu}$ are chosen so that $\chi\left(\eta_{\nu}\right)=1$, the $\chi_{\nu}$ are to have the natural support properties associated to these estimates, i.e.,

$$
\chi_{\nu}(\eta)=0 \text { if }|\eta|=1 \text { and }\left|\eta-\eta_{\nu}\right| \geqslant C \lambda^{-1 / 2} \text {. }
$$

Using the $\chi_{\nu}$ we make an angular decomposition of the operators as in [28] by setting

$$
\mathscr{F}_{\lambda}^{\nu} f(z)=\int e^{i \varphi(z, \eta)} \chi_{\nu}(\eta) a_{\lambda}(z, \eta) \hat{f}(\eta) d \eta .
$$

Having made an angular decomposition, we must now make a corresponding radial decomposition of the symbol. To this end, we fix $\rho \in C_{0}^{\infty}((-1,1))$ 
satisfying $\sum_{j \in \mathbb{Z}} \rho(u-j) \equiv 1$ and set

$$
\mathscr{F}_{\lambda}{ }^{\nu, j} f(z)=\int e^{i \varphi(z, \eta)} a_{\lambda}^{\nu, j}(z, \eta) \hat{f}(\eta) d \eta,
$$

with

$$
a_{\lambda}^{\nu, j}(z, \eta)=\chi_{\nu}(\eta) \rho\left(\lambda^{-1 / 2}|\eta|-j\right) a_{\lambda}(z, \eta) .
$$

Observe that if

$$
\mathscr{Q}_{\nu, j}=\operatorname{supp}_{\eta} a_{\lambda}^{\nu, j}
$$

then the $\mathscr{Q}_{\nu, j}$ are all comparable to cubes of sidelength $\lambda^{1 / 2}$ contained in the annulus $\{\eta:|\eta| \approx \lambda\}$. This, along with the fact that the symbols satisfy the natural estimates associated to this support property, i.e.,

$$
\left|\partial_{z}^{\gamma} \partial_{\eta}^{\alpha} a_{\lambda}^{\nu, j}(z, \eta)\right| \leqslant C_{\alpha, \gamma}(1+|\eta|)^{-|\alpha| / 2} \approx \lambda^{-|\alpha| / 2}
$$

makes the decomposed operators $\mathscr{F}_{\lambda}{ }^{\nu, j}$ much easier to analyze than the original one $\mathscr{F}_{\lambda}$.

The square function that will be used in the proof of (4.6) will involve operators which are related to the operators $\mathscr{F}_{\lambda}^{\nu, j}$. The main step argument is to establish the following

Proposition 4.5. Fix $\varepsilon>0$. Then, given $N$, there are finite constants $M(N)$ and $C_{N}$ so that whenever $Q \subset \mathbb{R}^{4}$ is a cube of sidelength $\lambda^{-1 / 2-\varepsilon}$

$$
\left\|\mathscr{F}_{\lambda} f\right\|_{L^{4}(Q)} \leqslant C_{N}\left(\lambda^{1 / 4+\varepsilon / 4} \sum_{|k| \leqslant M(N)}\left\|\left(\sum_{\nu, j}\left|\mathscr{F}_{\lambda, k}^{\nu, j} f\right|^{2}\right)^{1 / 2}\right\|_{L^{4}(Q)}+\lambda^{-N}\|f\|_{L^{4}\left(\mathbb{R}^{3}\right)}\right) .
$$

Here

$$
\mathscr{F}_{\lambda, k}^{\nu, j} f(z)=\int e^{i \varphi(z, \eta)} a_{\lambda, k}^{\nu, j}(z, \eta) \hat{f}(\eta) d \eta,
$$

with symbols satisfying

$$
\begin{gathered}
\operatorname{supp}_{\eta} a_{\lambda, k}^{\nu, j} \subset \mathscr{Q}_{\nu, j} \\
\left|\partial_{\eta}^{\alpha} a_{\lambda, k}^{\nu, j}(z, \eta)\right| \leqslant C_{\alpha} \lambda^{-|\alpha| / 2} \quad \forall \alpha .
\end{gathered}
$$

If the phase function $\varphi$ is fixed, the constants in (4.10) and (4.11) depend only on finitely many of those in (4.9).

The first operator $\mathscr{F}_{\lambda, 0}^{\nu, j}$ will just be an oscillatory factor times $\mathscr{F}_{\lambda}^{\nu, j}$, while, for $k \geqslant 1$, the operators in (4.10) will involve derivatives of the symbol and the phase function. The proposition is a variation of a square function estimate in Bourgain [5], relying on a somewhat different proof. 
Proof. It is clear that (4.10) would be a consequence of the following uniform upper bounds valid for $z \in Q$ :

$$
\begin{aligned}
\left\|\mathscr{F}_{\lambda} f\right\|_{L^{4}(Q)} & \leqslant C_{N}\left(|Q|^{1 / 4} \lambda^{1 / 4+\varepsilon / 4} \sum_{|k| \leqslant M(N)}\left(\sum_{\nu, j}\left|\mathscr{F}_{\lambda, k}^{\nu, j} f(z)\right|^{2}\right)^{1 / 2}+\lambda^{-N}\|f\|_{L^{4}\left(\mathbb{R}^{2}\right)}\right) \\
& =C_{N}\left(\lambda^{-1 / 4} \sum_{|k| \leqslant M(N)}\left(\sum_{\nu, j}\left|\mathscr{F}_{\lambda, k}^{\nu, j} f(z)\right|^{2}\right)^{1 / 2}+\lambda^{-N}\|f\|_{L^{4}\left(\mathbb{R}^{2}\right)}\right) .
\end{aligned}
$$

We may assume that $0 \in Q$ and we shall prove the estimate for $z=0$.

To proceed, given $\nu, j$ for which $\mathscr{Q}_{\nu, j}$ is nonempty, we choose $\eta^{\nu, j} \in \mathscr{Q}_{\nu, j}$ and set

$$
c_{\lambda}^{\nu, j}(z)=\int e^{i\left[\varphi(z, \eta)-\varphi\left(z, \eta^{\nu, j}\right)\right]} a_{\lambda}^{\nu, j}(z, \eta) \hat{f}(\eta) d \eta
$$

Note that

$$
\left|\partial_{z}^{\alpha}\left\{e^{i\left[\varphi(z, \eta)-\varphi\left(z, \eta^{\nu, j}\right)\right]} a_{\lambda}^{\nu, j}(z, \eta)\right\}\right|=O\left(\lambda^{|\alpha| / 2}\right) .
$$

So, if we let

$$
c_{\lambda, k}^{\nu, j}(z)=\partial_{z}^{k} c_{\lambda}^{\nu, j}(z), \quad k=\left(k_{1}, \ldots, k_{4}\right)
$$

it follows that

$$
c_{\lambda}^{\nu, j}(z)=\sum_{|k| \leqslant M(N)} z^{k} c_{\lambda, k}^{\nu, j}(0)+R_{M}(z)
$$

where if $z \in Q$ (and hence $|z|<\lambda^{-1 / 2-\varepsilon}$ ) and if $M$ is large enough,

$$
\left|R_{M}(z)\right| \leqslant C_{N} \lambda^{-N}\|f\|_{4} \text {. }
$$

Therefore, if we set

$$
\mathscr{F}_{\lambda, k}^{\nu, j} f(z)=\lambda^{-|k| / 2} \partial_{z}^{k} \int e^{i\left[\varphi(z, \eta)-\varphi\left(z, \eta^{\nu, j}\right)\right]} a_{\lambda}^{\nu, j}(z, \eta) \hat{f}(\eta) d \eta
$$

then since $\left|z^{k}\right| \lambda^{|k| / 2} \leqslant 1$, the left-hand side of (4.12) is dominated by $C \lambda^{-N}\|f\|_{4}$ plus

$$
\sum_{|k| \leqslant M}\left\|\sum_{\nu, j} e^{i \varphi\left(z, \eta^{\nu, j}\right)} \mathscr{F}_{\lambda, k}^{\nu, j} f(0)\right\|_{L^{4}(Q)} .
$$

Since $\left|\eta-\eta^{\nu, j}\right| \leqslant C \lambda^{1 / 2}$ for $\eta \in \operatorname{supp}_{\eta} a_{\lambda}^{\nu, j}$, it is clear that the symbol of $\mathscr{F}_{\lambda}^{\nu, j}$ satisfies (4.11). Therefore, by the last majorization, we would be done if we had the following discrete version of the $L^{2} \rightarrow L^{4}$ local smoothing theorem.

Lemma 4.6. Let $Q$ and $\eta^{\nu, j}$ be as above. Then if $Q$ is contained in a small relatively compact neighborhood of $\operatorname{supp}_{\eta} a_{\lambda}$ (so that $\varphi$ is well defined)

$$
\left\|\sum_{\nu, j} e^{i \varphi\left(z, \eta^{\nu, j}\right)} c^{\nu, j}\right\|_{L^{4}(Q)} \leqslant C \lambda^{-1 / 4}\left(\sum_{\nu, j}\left|c^{\nu, j}\right|^{2}\right)^{1 / 2} .
$$


Proof. As before we assume $0 \in Q$. If we then normalize the phase function by setting

$$
\phi(z, \eta)=\varphi(z, \eta)-\varphi(0, \eta)
$$

then the desired estimate is equivalent to

$$
\lambda^{1 / 4}\left\|\sum_{\nu, j} e^{i \phi\left(z, \eta^{\nu, j}\right)} c^{\nu, j}\right\|_{L^{4}(Q)} \leqslant C\left(\sum_{\nu, j}\left|c^{\nu, j}\right|^{2}\right)^{1 / 2} .
$$

To see that this is a corollary of the $L^{2} \rightarrow L^{4}$ local smoothing theorem, we let $Q_{\lambda}^{\nu, j}$ be the cube of sidelength $\lambda^{1 / 2}$ in $\mathbb{R}^{3}$ which is centered at $\eta^{\nu, j}$. If $\lambda$ is sufficiently large and if we let

$$
b_{\lambda}^{\nu, j}(z, \eta)=\left\{\int_{Q_{\lambda}^{\nu, j}} e^{i\left[\phi(z, \eta)-\phi\left(z, \eta^{\nu, j}\right)\right]} d \eta\right\}^{-1} \cdot \chi_{Q_{\lambda}^{\nu, j}}(\eta),
$$

it follows that, for $z \in Q$,

$$
\left|b_{\lambda}^{\nu, j}(z, \eta)\right| \leqslant C\left|Q_{\lambda}^{\nu, j}\right|^{-1}=C^{\prime} \lambda^{-3 / 2} .
$$

To see this, note that $\phi(z, \eta)-\phi\left(z, \eta^{\nu, j}\right)=0$ when $\eta=\eta^{\nu, j}$ and $\nabla_{\eta} \phi(z, \eta)=$ $O\left(\lambda^{-1 / 2-\varepsilon}\right)$ for $z$ in $Q$. Hence, $\left|\phi(z, \eta)-\phi\left(z, \eta^{\nu, j}\right)\right|<1 / 2$ for large $\lambda$, giving the estimate. Similar considerations show that

$$
\left|\partial_{z}^{\alpha} b_{\lambda}^{\nu, j}(z, \eta)\right| \leqslant C_{\alpha} \lambda^{-3 / 2} \lambda^{|\alpha| / 2}, \quad z \in Q
$$

These estimates are relevant since the quantity inside the $L^{4}$ norm in (4.14) equals

$$
\int e^{i \phi(z, \eta)} \sum_{\nu, j} b_{\lambda}^{\nu, j}(z, \eta) c^{\nu, j} d \eta
$$

So if we argue as in [33] and write

$$
\begin{aligned}
b_{\lambda}^{\nu, j}(z, \eta)= & b_{\lambda}^{\nu, j}(0, \eta)+\int_{0}^{z_{1}} \frac{\partial}{\partial u_{1}} b_{\lambda}^{\nu, j}\left(u_{1}, 0, \ldots, 0, \eta\right) d u_{1}+\cdots \\
& +\int_{0}^{z_{1}} \cdots \int_{0}^{z_{4}} \frac{\partial}{\partial u_{1}} \cdots \frac{\partial}{\partial u_{4}} b_{\lambda}^{\nu, j}(u, y) d u
\end{aligned}
$$

it follows that the left-hand side of (4.14) is dominated by

$$
\begin{aligned}
& \lambda^{1 / 4}\left\|\int e^{i \phi(z, \eta)} \sum_{\nu, j} b_{\lambda}^{\nu, j}(0, \eta) c^{\nu, j} d \eta\right\|_{L^{4}(Q)}+\cdots \\
& +\lambda^{1 / 4} \int_{-\lambda^{-1 / 2-\varepsilon}}^{\lambda^{-1 / 2-\varepsilon}} \cdots \int_{-\lambda^{-1 / 2-\varepsilon}}^{\lambda^{-1 / 2-\varepsilon}}\left\|\int e^{i \phi(z, \eta)} \sum_{\nu, j} \frac{\partial}{\partial u_{1}} \cdots \frac{\partial}{\partial u_{4}} b_{\lambda}^{\nu, j}(u, \eta) c^{\nu, j} d \eta\right\|_{L^{4}(Q)} d u .
\end{aligned}
$$

Since $b_{\lambda}^{\nu, j}$ vanishes unless $|\eta| \approx \lambda$, Theorem 3.1 and Plancherel's theorem imply that this is majorized by

$$
\begin{aligned}
& \lambda^{1 / 4} \lambda^{1 / 2}\left\|\sum_{\nu, j} b_{\lambda}^{\nu, j}(0, \eta) c^{\nu, j}\right\|_{L^{2}(d \eta)}+\cdots \\
& \quad+\lambda^{1 / 4} \lambda^{1 / 2} \int_{-\lambda^{-1 / 2-\varepsilon}}^{\lambda^{-1 / 2-\varepsilon}} \cdots \int_{-\lambda^{-1 / 2-\varepsilon}}^{\lambda^{-1 / 2-\varepsilon}}\left\|\sum_{\nu, j} \frac{\partial}{\partial u_{1}} \cdots \frac{\partial}{\partial u_{4}} b_{\lambda}^{\nu, j}(u, \eta) c^{\nu, j}\right\|_{L^{2}(d \eta)} d u
\end{aligned}
$$


however, using (4.15) and the fact that the sets $Q_{\lambda}^{\nu, j}$ have finite overlap shows that this in turn is

$$
\leqslant C \lambda^{1 / 4} \lambda^{1 / 2} \lambda^{-3 / 2}\left(\sum_{\nu, j}\left|c^{\nu, j}\right|^{2}\left|Q_{\lambda}^{\nu, j}\right|\right)^{1 / 2}=C^{\prime}\left(\sum_{\nu, j}\left|c^{\nu, j}\right|^{2}\right)^{1 / 2}
$$

as desired.

Returning to the proof of (4.6), let us fix a lattice of cubes of sidelength $\lambda^{-1 / 2-\varepsilon}$. Then, if we raise (4.10) to the 4 th power and then sum over the cubes in the lattice which intersect with $\operatorname{supp}_{z} a_{\lambda}$, we conclude that, for large enough $M$,

$$
\left\|\mathscr{F}_{\lambda} f\right\|_{L^{4}\left(\mathbb{R}^{4}\right)} \leqslant C \lambda^{1 / 4+\varepsilon} \sum_{|k| \leqslant M}\left\|\left(\sum_{\nu, j}\left|\mathscr{F}_{\lambda, k}^{\nu, j} f\right|^{2}\right)^{1 / 2}\right\|_{L^{4}\left(\mathbb{R}^{4}\right)}+C\|f\|_{L^{4}\left(\mathbb{R}^{3}\right)} .
$$

To apply this we need an estimate about the kernel

$$
K_{\lambda, k}^{\nu, j}(z, y)=\int e^{i[\varphi(z, \eta)-\langle y, \eta\rangle]} a_{\lambda, k}^{\nu, j}(z, \eta) d \eta
$$

of $\mathscr{F}_{\lambda, k}^{\nu, j}$. If $\mathscr{N} \subset \mathbb{R}^{4} \times \mathbb{R}^{3} \backslash 0$ is a small conic neighborhood of $\operatorname{supp} a_{\lambda}$, we define the smooth curves

$$
\gamma_{y, \eta}=\left\{z: \varphi_{\eta}^{\prime}(z, \eta)=y,(z, \eta) \in \mathscr{N}\right\} .
$$

Then we have the following pointwise estimate.

Lemma 4.7. If $\eta_{\nu}$ are the unit vectors occurring in the definition of $\mathscr{F}_{\lambda}^{\nu}$, then, given any $N$,

$$
\left|K_{\lambda, k}^{\nu, j}(z, y)\right| \leqslant C_{k, N} \lambda^{3 / 2}\left(1+\lambda^{1 / 2} \operatorname{dist}\left(z, \gamma_{y, \eta_{n}}\right)\right)^{-N}
$$

Proof. We may assume that $\operatorname{supp}_{z} a_{\lambda}$ is small. Then local coordinates $z=$ $(x, t)$ can be chosen so that $\operatorname{det} \varphi_{x \eta}^{\prime \prime} \neq 0$ and $\varphi_{t}^{\prime} \neq 0$. Since the latter implies $\varphi_{t \eta}^{\prime \prime} \neq 0$, it follows from the implicit function theorem that $\gamma_{y, \eta_{\nu}}$ is of the form $(x(t), t)$ near $\operatorname{supp}_{z} a_{\lambda}$ if this set is small enough. So, under this assumption there must be a $c>0$ so that

$$
\left|\nabla_{\eta}[\varphi(z, \eta)-\langle y, \eta\rangle]\right| \geqslant c|x-x(t)|, \quad z=(x, t) \in \operatorname{supp}_{z} a_{\lambda}, \quad \eta=\eta_{\nu} .
$$

But $\nabla_{\eta} \varphi$ is homogeneous of degree zero in $\eta$ and therefore

$$
\left|\nabla_{\eta}\left[\varphi(z, \eta)-\varphi\left(z, \eta_{\nu}\right)\right]\right| \leqslant C \lambda^{-1 / 2}, \quad \eta \in \mathscr{Q}_{\nu, j} .
$$

Combining these two bounds implies that there is a $c^{\prime}>0$ so that

$$
\left|\nabla_{\eta}[\varphi(z, \eta)-\langle y, \eta\rangle]\right| \geqslant c^{\prime} \operatorname{dist}\left(z, \gamma_{y, \eta_{\nu}}\right) \text {, }
$$

provided that $\operatorname{dist}\left(z, \gamma_{y, \eta_{\nu}}\right)$ is larger than a fixed multiple of $\lambda^{-1 / 2}$. Consequently, if we recall (4.11), we see that (4.19) follows from integrating by parts in (4.17). 
In particular, we obtain the uniform bounds

$$
\int\left|K_{\lambda, k}^{\nu, j}(z, y)\right| d y \leqslant C \text {. }
$$

In order to apply this we define for $m \in \mathbb{Z}^{3}$ the operators $P^{m}$ via their Fourier transforms by

$$
\begin{aligned}
\widehat{P^{m}} f(\eta) & =\rho\left(\lambda^{-1 / 2} \eta-m\right) \hat{f}(\eta) \\
& \equiv \rho\left(\lambda^{-1 / 2} \eta_{1}-m_{1}\right) \rho\left(\lambda^{-1 / 2} \eta_{2}-m_{2}\right) \rho\left(\lambda^{-1 / 2} \eta_{3}-m_{3}\right) \hat{f}(\eta),
\end{aligned}
$$

where $\rho$ is the function occurring in the definition of $\mathscr{F}_{\lambda}^{\nu, j}$. Then $\sum_{m \in \mathbb{Z}^{3}} P^{m} f$ $=f$. In addition, if for a given $\nu, j$ we let $\mathscr{I}_{\nu, j} \subset \mathbb{Z}^{3}$ be those $m$ for which $\mathscr{Q}_{\nu, j} \cap \operatorname{supp} \rho\left(\lambda^{-1 / 2} \eta-m\right) \neq \varnothing$, it follows that

$$
\text { Card } \mathscr{I}_{\nu, j} \leqslant C
$$

since the $\mathscr{Q}_{\nu, j}$ are comparable to cubes of sidelength $\lambda^{1 / 2}$. Also, since the sets $\mathscr{Q}_{\nu, j}$ have finite overlap, there is an absolute constant $C$ so that

$$
\text { Card }\left\{(\nu, j): m \in \mathscr{I}_{\nu, j}\right\} \leqslant C \quad \forall m \in \mathbb{Z}^{3} .
$$

We now use the uniform bounds (4.20) and apply the Schwarz inequality along with (4.11) and (4.21) and obtain

$$
\begin{aligned}
\left|\mathscr{F}_{\lambda, k}^{\nu, j} f(z)\right|^{2} & \leqslant C \int\left|\sum_{m \in \mathcal{F}_{\nu, j}} P^{m} f(y)\right|^{2}\left|K_{\lambda, k}^{\nu, j}(z, y)\right| d y \\
& \leqslant C^{\prime} \int \sum_{m \in \mathcal{F}_{\nu, j}}\left|P^{m} f(y)\right|^{2}\left|K_{\lambda, k}^{\nu, j}(z, y)\right| d y .
\end{aligned}
$$

If we now use (4.22), we see that for a given nonnegative $L^{2}$ function $g(z)$

$$
\begin{aligned}
& \int \sum_{\nu, j}\left|\mathscr{F}_{\lambda, k}^{\nu, j} f(z)\right|^{2} g(z) d z \\
& \quad \leqslant C \int \sum_{m}\left|P^{m}(y)\right|^{2} \sup _{\nu, j}\left\{\int\left|K_{\lambda, k}^{\nu, j}(z, y)\right| g(z) d z\right\} d y .
\end{aligned}
$$

In order to finish the proof we shall use the following square function estimate

$$
\left\|\left(\sum_{m}\left|P^{m} f\right|^{2}\right)^{1 / 2}\right\|_{p} \leqslant C\|f\|_{p} \quad \text { if } 2 \leqslant p \leqslant \infty .
$$

It is due to Carleson and Córdoba [13], and a simple proof due to Rubio de Francia is contained in [16]. By (4.16), (4.22), (4.23), and the inverse Hölder inequality we would be done if we could prove

$$
\left(\int_{\mathbb{R}^{3}} \sup _{\nu, j}\left|\int\right| K_{\lambda, k}^{\nu, j}(z, y)|g(z) d z|^{2} d y\right)^{1 / 2} \leqslant C \lambda^{1 / 4}\|g\|_{L^{2}\left(\mathbb{R}^{4}\right)},
$$


but this follows from estimates for variable coefficient Kakeya maximal functions whose proof we postpone until the next section (cf. Theorem 5.2).

Remarks on sharp local smoothing for operators of type $(1 / 2,1 / 2)$. Suppose that $\operatorname{dim} X=\operatorname{dim} Y=n$ and let

$$
\mathscr{F} f(x)=\int_{\mathbb{R}^{n}} e^{i \varphi(x, \eta)} a(x, \eta) \hat{f}(\eta) d \eta
$$

be a Fourier integral operator of order $\mu-1 / 4$ and type $(1 / 2,1 / 2)$. That is, we assume that $\varphi$ is a generating function for a canonical relation $\mathscr{C}$ and that the symbol satisfies

$$
\left|\partial_{x}^{\gamma} \partial_{\eta}^{\alpha} a(z, \eta)\right| \leqslant C_{\alpha \gamma}(1+|\eta|)^{\mu-|\alpha| / 2+|\gamma| / 2} .
$$

Then it was shown in $[1,17,28]$, that for $1<p<\infty$

$$
\mathscr{F} f \in L_{\mathrm{loc}}^{p}(X) \text { if } f \in L^{p}(Y) \text { and } \mu \leqslant-\left(n-\frac{1}{2}\right)\left|\frac{1}{2}-\frac{1}{p}\right| \text {. }
$$

Moreover, in general this result cannot be improved.

However, if we consider operators from $Y$ to $Z$ satisfying the cinematic curvature hypothesis, then we shall see that there is a range of exponents $p>$ 2 for which there is local smoothing of all orders $<1 / p$. In general such an operator cannot send $L^{p}(Y) \rightarrow L_{\mathrm{loc}}^{p}(Z)$ if the order of the symbol is $>-\left(n-\frac{1}{2}\right)\left|\frac{1}{2}-\frac{1}{p}\right|+\frac{1}{p}$. For if one works locally, writing $z=(x, t)$, an application of the Sobolev embedding theorem would give $\sup _{t \in I}|\mathscr{F}(x, t)| \in L_{\text {loc }}^{p}(X)$ if $f \in L^{p}(Y)$, which is stronger than (4.28).

Theorem 4.8. Suppose that $\mathscr{F} \in I_{1 / 2}^{\mu-1 / 4}(Z, Y ; \mathscr{C})$ is of the form (4.26) (with $x$ replaced by $z$ ). Suppose also that $\mathscr{C}$ satisfies the nondegeneracy assumption (2.2), (2.3) and the cone condition (2.6). Then, if $\mu<-\left(n-\frac{1}{2}\right)\left(\frac{1}{2}-\frac{1}{p}\right)+\frac{1}{p}$, it follows that $\mathscr{F} f \in L_{\mathrm{loc}}^{p}(Z)$ if $f \in L^{p}(Y)$ and

(1) $6 \leqslant p<\infty \quad n=2$;

(2) $4 \leqslant p<\infty \quad n \geqslant 3$.

The proof is a straightforward modification of the proof of Theorem 4.2. If we assume now that $a(z, \eta)$ is a symbol of order 0 and type $(1 / 2,1 / 2)$ and if we set $a_{\lambda}(z, \eta)=\beta(|\eta| / \lambda)$, then we must show that the associated dyadic operators satisfy the following estimates for $\varepsilon>0$ and $p$ as in the theorem:

$$
\left\|\mathscr{F}_{\lambda} f\right\|_{p} \leqslant C_{\varepsilon} \lambda^{\left(n-\frac{1}{2}\right)\left(\frac{1}{2}-\frac{1}{p}\right)-\frac{1}{p}+\varepsilon}\|f\|_{p} .
$$

As before, we prove this by first decomposing the operators into pieces of the form

$$
\mathscr{F}_{\lambda}^{\nu} f(z)=\int e^{i \varphi(z, \eta)} \chi_{\nu}(\eta) a_{\lambda}(z, \eta) \hat{f}(\eta) d \eta,
$$

where the $\chi_{\nu}$ are the $n$-dimensional versions of the homogeneous cutoffs that were used before. We then make a further decomposition into operators $\mathscr{F}_{\lambda}{ }^{\nu, j}$ with symbols given by (4.7). Then, as before, $\mathscr{Q}_{\nu, j}=\operatorname{supp}_{\eta} a_{\lambda}^{\nu, j}$ is comparable to a $\lambda^{1 / 2}$ cube which lies in the annulus $\{\eta:|\eta| \approx \lambda\}$. 
Having set up the notation, we need the following $n$-dimensional version of Lemma 4.6: If $Q \subset \mathbb{R}^{n+1}$ is a cube of sidelength $\lambda^{-1 / 2-\varepsilon}$ and if $\eta^{\nu, j} \in \mathscr{Q}_{\nu, j}$, then there is a uniform constant $C$ such that for large $\lambda$

$$
\begin{aligned}
\left\|\sum_{\nu, j} e^{i \varphi\left(z, \eta^{\nu, j}\right)} c^{\nu, j}\right\|_{L^{p}(Q)} \leqslant C \lambda^{-\frac{n}{4}+n\left(\frac{1}{2}-\frac{1}{p}\right)-\frac{1}{p}}\left(\sum_{\nu, j}\left|c^{\nu, j}\right|^{2}\right)^{1 / 2}, \\
p \geqslant \frac{2(n+1)}{n-1} .
\end{aligned}
$$

Since we are assuming that $a$ is a symbol of order 0 and type $(1 / 2,1 / 2)$ the estimate (4.13) holds here as well. So the argument which led to (4.12) gives that, for $p$ as in (4.30),

$$
\begin{aligned}
\left\|\mathscr{F}_{\lambda} f\right\|_{L^{p}(Q)} \leqslant & C \lambda^{-\frac{n}{4}+n\left(\frac{1}{2}-\frac{1}{p}\right)-\frac{1}{p}}|Q|^{-\frac{1}{p}} \sum_{|k| \leqslant M(N)}\left\|\left(\sum_{\nu, j}\left|\mathscr{F}_{\lambda, k}^{\nu, j} f\right|^{2}\right)^{1 / 2}\right\|_{L^{p}(Q)} \\
& +C_{N} \lambda^{-N}\|f\|_{L^{p}(Y)} .
\end{aligned}
$$

However, if $n \geqslant 3$ and if we apply the Kakeya maximal estimate (Theorem 5.2) just like before, this leads to (4.29) in the special case of $p=4$. The other cases follow from interpolating with the estimate for $p=\infty$ which was proved in $[28]$.

To prove (4.29) for $n=2$ we need to use the fact that, for $1 \leqslant q \leqslant 2$, the Kakeya maximal operator in (4.25) has an $L^{q}$ operator norm which is $O\left(\delta^{(1-2 / p)}|\log \delta|^{1-1 / p}\right)$. This just follows from interpolating between the $L^{2}$ estimate (Theorem 5.1) and the trivial $L^{1}$ estimate that the maximal operators are bounded on $L^{1}$ with norm $O\left(\delta^{-1}\right)$. Using this estimate for $q=3 / 2$ leads to (4.29) when $p=6$, which as above leads to the full two-dimensional result by interpolation.

\section{VARIABLE COEFFICIENT KAKEYA MAXIMAL FUNCTIONS}

The purpose of this section is to prove a sharp $L^{2}$ estimate for variable coefficient versions of the Kakeya maximal function, as needed in (4.25).

We now assume that $Z$ and $Y$ are as in $\S 2$ with the dimension of $Y$ being $n \geqslant 2$ and $\operatorname{dim} Z=n+1$. To state the hypotheses in an invariant way, let $\mathscr{C}$ be as in $\S 2$. Then we set for $(y, \eta) \in \Pi_{T^{*} Y}(\mathscr{C})$

$$
\gamma_{y, \eta}=\{z \in Z:(z, \zeta, y, \eta) \in \mathscr{C}, \text { some } \zeta\} .
$$

It follows from our nondegeneracy assumptions (2.2) and (2.3) that $\gamma_{y, \eta}$ is a $C^{\infty}$ immersed curve in $Z$ which depends smoothly on the parameters $(y, \eta)$. Note that if $\mathscr{C}$ is as in (2.7), then

$$
\gamma_{y, \eta}=\left\{z \in Z: \varphi_{\eta}^{\prime}(z, \eta)=y\right\} .
$$

Let us fix a smooth metric on $Z$ and put

$$
R_{y, \eta}^{\delta}=\left\{z: \operatorname{dist}\left(z, \gamma_{y, \eta}\right)<\delta\right\} .
$$


If $0<\delta<1 / 2$ and $\alpha \in C_{0}^{\infty}(Y \times Z)$ we set

$$
\mathscr{M}_{\delta} g(y)=\sup _{\eta \in \Pi_{T_{y}^{*} Y}(\mathscr{C})}\left|\frac{1}{\operatorname{Vol}\left(R_{y, \eta}^{\delta}\right)} \int_{R_{y, \eta}^{\delta}} \alpha(y, z) g(z) d z\right| .
$$

Then we have the following results.

Theorem 5.1. Suppose $\operatorname{dim} Z=3, \operatorname{dim} Y=2$ and suppose that $\mathscr{C}$ satisfies the nondegeneracy conditions (2.2) and (2.3) as well as the cone condition (2.6). Then

$$
\left\|\mathscr{M}_{\delta} g\right\|_{L^{2}(Y)} \leqslant C|\log \delta|^{1 / 2}\|g\|_{L^{2}(Z)} .
$$

In higher dimensions we have

Theorem 5.2. Suppose $n \geqslant 3, \operatorname{dim} Z=n+1, \operatorname{dim} Y=n$ and suppose that $\mathscr{C}$ satisfies the nondegeneracy conditions (2.2) and (2.3) and the cone condition (2.6). Then

$$
\left\|\mathscr{M}_{\delta} g\right\|_{L^{2}(Y)} \leqslant C \delta^{-(n-2) / 2}\|g\|_{L^{2}(Z)} .
$$

Since the canonical relation associated to the operator in the proof of Theorem 4.2 is given by $(2.7)$, it is clear that the maximal function in (4.25) is majorized by $C_{N} \sum_{j \geqslant 0} 2^{-j N} \mathscr{M}_{2^{j} \lambda^{-1 / 2}} g$ and, therefore, (5.4) implies (4.25).

Before turning to the proof, let us state one more consequence. If $(X, g)$ is a compact Riemannian manifold, then for a given $y \in X$ and $\theta \in T_{y} X$, let $\gamma_{y, \theta}(t)$ be the geodesic starting at $y$ in the direction $\theta$ which is parameterized by arclength. Then if $T X \ni(y, \theta) \rightarrow(y, \eta(y, \theta)) \in T^{*} X$ is the Legendre transform, it follows that $\gamma_{y, \theta}$ coincides with $\gamma_{y, \eta(y, \theta)}$ in (5.1) if $\mathscr{C}$ is the canonical relation (2.13) with $q(x, \xi)=\sqrt{\sum g^{j k}(x) \xi_{j} \xi_{k}}$ being the square root of the cometric and $\chi_{t}$ being the flowout along the Hamilton vector field associated to $q$ (see [37]). So, if we fix $0<T<\infty$ and let

$$
R_{y, \theta}^{\delta, T}=\left\{(x, t): \operatorname{dist}\left(x, \gamma_{x, \theta}(t)\right)<\delta, \quad 0 \leqslant t \leqslant T\right\},
$$

a special case of our results is

$$
\begin{aligned}
& \left\|\sup _{\theta \in S_{y}^{*} X} \frac{1}{\operatorname{Vol}\left(R_{y, \theta}^{\delta, T}\right)} \int_{R_{y, \theta}^{\delta, T}}|g(z)| d z\right\|_{L^{2}(X)} \\
& \quad \leqslant C_{T} \max \left\{\delta^{-\frac{n-2}{2}},|\log \delta|^{1 / 2}\right\}\|g\|_{L^{2}(X \times \mathbb{R})} .
\end{aligned}
$$

This is of course stronger than the inequality (1.2) stated in the introduction since it involves averages over small neighborhoods of geodesic light rays rather than just small neighborhoods of geodesics. Let us remark that the sharpness of the constants in (5.4) and (5.5) can be checked as in the Euclidean case by testing $\mathscr{M}_{\delta}$ against functions which are radial in an appropriate coordinate system. For example, in two dimensions one takes $g_{\delta}(x, t)=|x|^{-1 / 2}$, if $\delta \leqslant|x| \leqslant 1 / 2$, $0 \leqslant t \leqslant 1$, and $g_{\delta}(x, t)=0$ elsewhere. 
We shall first give a proof of Theorem 5.1 and then describe the modifications which are needed in the higher-dimensional case. At the end of the section we shall consider a nonisotropic variant which will be needed in the next section.

Proof of Theorem 5.1. We may work locally and assume that $\gamma_{y, \eta}$ is of the form (5.2) where $\eta /|\eta|$ is close to $(1,0)$.

To proceed, we fix a $C_{0}^{\infty}$ function $a$ satisfying $\hat{a} \geqslant 0$. Then let $\alpha(z, \vartheta) \in$ $C_{0}^{\infty}$ supported in a small neighborhood of $z=0, \vartheta=0$, say where $|\vartheta|<\varepsilon_{0}<$ $\pi / 4$. Then we put

$$
\alpha_{\delta}(z, \vartheta ; \eta)=\alpha(z, \vartheta) a(\delta \eta)
$$

and

$$
\phi(z, \eta, \vartheta)=\left\langle\varphi_{\eta}^{\prime}(z, \cos \vartheta, \sin \vartheta), \eta\right\rangle
$$

and define

$$
A_{\delta} g(y, \vartheta)=\int_{\mathbb{R}^{3}} \int_{\mathbb{R}^{2}} e^{i(\phi(z, \eta, \vartheta)-\langle y, \eta\rangle)} \alpha_{\delta}(z, \eta, \vartheta) g(z) d \eta d z .
$$

Clearly it suffices to prove the $L^{2}$ estimate for the maximal function $\sup _{\vartheta}\left|A_{\delta} g(y, \vartheta)\right|$. We consider $A_{\delta}$ as a Fourier integral operator sending functions of $z$ into functions of $(y, \vartheta)$. We shall see that this Fourier integral operator becomes degenerate if $(\cos \vartheta, \sin \vartheta)= \pm \eta /|\eta|$, in the sense that the associated canonical relation is not locally the graph of a symplectic diffeomorphism. We may write $\alpha_{\delta}$ as the sum of two symbols, say $\alpha_{\delta}^{1}, \alpha_{\delta}^{2}$, with the property that if $(\cos \vartheta, \sin \vartheta)=\eta /|\eta|$ then $\alpha_{\delta}^{2}(z, \eta, \vartheta)=0$ and if $(\cos \vartheta, \sin \vartheta)=-\eta /|\eta|$ then $\alpha_{\delta}^{1}(z, \eta, \vartheta)=0$. Both cases can be handled in the same way and, therefore, it is no loss of generality to assume in what follows that

$$
\alpha_{\delta}(z, \eta, \vartheta)=0 \quad \text { if }|(\cos \vartheta, \sin \vartheta)-\eta /| \eta|| \geqslant 3 / 2 .
$$

It will be useful to break up the operators $A_{\delta}$ by means of a dyadic decomposition of the symbol. To do this, as before, we let $\beta \in C_{0}^{\infty}(\mathbb{R} \backslash 0)$ satisfy $\sum_{k} \beta\left(2^{-k} s\right)=1, s \neq 0$ and define

$$
A_{\delta}^{j} g(y, \vartheta)=\iint e^{i(\phi(z, \eta, \vartheta)-\langle y, \eta\rangle)} \beta\left(2^{-j}|\eta|\right) \alpha_{\delta}(z, \eta, \vartheta) g(z) d \eta d z .
$$

It is trivial to check that the maximal function $\sup _{\vartheta}\left|A_{\delta}^{j} g(y, \vartheta)\right|$ defines a bounded operator from $L^{2}\left(\mathbb{R}^{3}\right)$ into $L^{2}\left(\mathbb{R}^{2}\right)$ with norms depending on $j$. Therefore it suffices to show that

$$
\left(\int_{\mathbb{R}^{2}} \sup _{\vartheta}\left|\sum_{2^{j}>\varepsilon_{0}^{-1}} A_{\delta}^{j} g(y, \vartheta)\right|^{2} d y\right)^{1 / 2} \leqslant C|\log \delta|^{1 / 2}\|g\|_{L^{2}\left(\mathbb{R}^{3}\right)} .
$$

In order to handle the degeneracy of the Fourier integral operator $A_{\delta}^{j}$ we introduce another decomposition. Write $\eta /|\eta|=(\cos \vartheta(\eta), \sin \vartheta(\eta))$ and let for $2 \leqslant 2^{k} \leqslant \varepsilon_{0} 2^{j}$

$$
\beta^{j, k}(\eta, \vartheta)=\beta\left(2^{k-j}|\vartheta-\vartheta(\eta)|\right) \beta\left(2^{-j} \eta\right)
$$


Moreover define $\beta^{j, 0}$ in the analogous way, such that $\beta^{j, 0}$ is supported where $\eta \approx 2^{j}$ and $|\vartheta-\vartheta(\eta)| \leqslant 2^{-j+1}$, and let

$$
\tilde{\beta}^{j}(\eta, \vartheta)=\beta\left(2^{-j} \eta\right)\left(1-\sum_{2^{k}<\varepsilon_{0} 2^{j}} \beta^{j, k}(\eta, \vartheta)\right) .
$$

Then we define operators

$$
\begin{aligned}
A_{\delta}^{j, k} g(y, \vartheta) & =\iint e^{i(\phi(z, \eta, \vartheta)-\langle y, \eta\rangle)} \beta^{j, k}(\eta, \vartheta) \alpha_{\delta}(z, \eta, \vartheta) g(z) d \eta d z, \\
\tilde{A}_{\delta}^{j} g(y, \vartheta) & =\iint e^{i(\phi(z, \eta, \vartheta)-\langle y, \eta\rangle)} \tilde{\beta}^{j}(\eta, \vartheta) \alpha_{\delta}(z, \eta, \vartheta) g(z) d \eta d z .
\end{aligned}
$$

The main inequalities we are going to use about these operators are

$$
\begin{aligned}
& 2^{k / 2}\left\|A_{\delta}^{j, k} g\right\|_{L^{2}}+2^{-k / 2}\left\|(\partial / \partial \vartheta) A_{\delta}^{j, k} g\right\|_{L^{2}} \\
& \quad \leqslant C_{N} \min \left\{1,\left(2^{-j} \delta^{-1}\right)^{-N}\right\}\|g\|_{L^{2}} \quad \text { if } k \geqslant j / 2, \\
& 2^{(j-k) / 2}\left\|A_{\delta}^{j, k} g\right\|_{L^{2}}+2^{(k-j) / 2}\left\|(\partial / \partial \vartheta) A_{\delta}^{j, k} g\right\|_{L^{2}} \\
& \quad \leqslant C_{N} \min \left\{1,\left(2^{-j} \delta^{-1}\right)^{-N}\right\}\|g\|_{L^{2}} \quad \text { if } k \leqslant j / 2,
\end{aligned}
$$

and

(5.10) $2^{j / 2}\left\|\tilde{A}_{\delta}^{j} g\right\|_{L^{2}}+2^{-j / 2}\left\|(\partial / \partial \vartheta) \tilde{A}_{\delta}^{j} g\right\|_{L^{2}} \leqslant C_{N} \min \left\{1,\left(2^{-j} \delta^{-1}\right)^{-N}\right\}\|g\|_{L^{2}}$.

Let us first show why (5.9) and (5.10) imply the desired maximal inequality. We use

$$
\begin{aligned}
(5.11) \sup _{\vartheta \in \mathbb{R}}|F(\vartheta)|^{2} \leqslant C\left(\int|F(s)|^{2} d s\right)^{1 / 2}\left(\int\left|F^{\prime}(s)\right|^{2} d s\right)^{1 / 2} \\
\text { if } F \in C^{1}(\mathbb{R}) \text { and } F(0)=0,
\end{aligned}
$$

which just follows from writing $(F(\vartheta))^{2}=2 \int_{0}^{\vartheta} F(s) F^{\prime}(s) d s$ and then applying Schwarz's inequality.

To apply this we set for positive integers $k$

$$
B_{\delta}^{k} g(y, \vartheta)=\sum_{2^{k} \leqslant \varepsilon_{0} 2^{j}} A_{\delta}^{j, k} g(y, \vartheta) .
$$

As in the proof of Theorem 3.1 let $L_{j}, L_{j}^{\prime}$ denote Littlewood-Paley operators acting on $z \in \mathbb{R}^{n+1}$ and $y \in \mathbb{R}^{n}$, respectively. Then there is a uniform constant $C_{0}$ such that for $2^{k} \leqslant \varepsilon_{0} 2^{j}$

$$
\begin{gathered}
L_{j^{\prime}} A_{\delta}^{j, k}=0 \text { if }\left|j-j^{\prime}\right| \geqslant 4, \\
\left\|A_{\delta}^{j, k} L_{j^{\prime}}^{\prime} g(\cdot, \vartheta)\right\|_{2} \leqslant C_{N} \min \left\{2^{-j N}, 2^{-j^{\prime} N}\right\}\|g\|_{2} \quad \text { if }\left|j-j^{\prime}\right| \geqslant C_{0} .
\end{gathered}
$$

The first statement follows from the disjoint support of the symbols if one uses the Fourier transform. For the second, one has to use the homogeneity of the 
phase function which implies that $\left|\left\langle\varphi_{\eta}^{\prime}(z, \theta), \eta\right\rangle\right| \approx|\eta|$ if $\theta$ is close to $\eta /|\eta|$ and, therefore,

$$
\left|\nabla_{z}\left(\phi(z, \eta, \vartheta)-\left\langle z, \eta^{\prime}\right\rangle\right)\right| \geqslant c \max \left\{2^{j}, 2^{j^{\prime}}\right\}
$$

if $|\eta| \approx 2^{j},\left|\eta^{\prime}\right| \approx 2^{j^{\prime}},\left|j-j^{\prime}\right| \geqslant C_{0}$ and $\vartheta(\eta)$ is close to $\vartheta$. Now (5.12) immediately follows by an integration by parts.

Next we apply (5.11) and (5.12) and see that

$$
\begin{aligned}
& \| \sup _{\vartheta} \mid \sum_{k} B_{\delta}^{k} g(\cdot, \vartheta) \mid \\
& \leqslant C|\log \delta|^{1 / 2}\left(\sum_{L^{2}\left(\mathbb{R}^{n}\right)}\left\|\sup _{\vartheta}\left|B_{\delta}^{k} g(\cdot, \vartheta)\right|\right\|_{L^{2}\left(\mathbb{R}^{n}\right)}^{2}\right)^{1 / 2} \\
& \leqslant C|\log \delta|^{1 / 2}\left(\sum_{k}\left\|B_{\delta}^{k} g\right\|_{L^{2}\left(\mathbb{R}^{n+1}\right)}\left\|(\partial / \partial \vartheta) B_{\delta}^{k} g\right\|_{L^{2}\left(\mathbb{R}^{n+1}\right)}\right)^{1 / 2} \\
& \leqslant C|\log \delta|^{1 / 2}\left[\left(\sum_{j, k} 2^{k / 2}\left\|A_{\delta}^{j, k} L_{j} g\right\|_{L^{2}\left(\mathbb{R}^{n+1}\right)}^{2}\right)^{1 / 2}\right. \\
&\left.\quad \times\left(\sum_{j, k} 2^{-k / 2}\left\|(\partial / \partial \vartheta) A_{\delta}^{j, k} L_{j} g\right\|_{L^{2}\left(\mathbb{R}^{n+1}\right)}^{2}\right)^{1 / 2}+\|g\|_{L^{2}\left(\mathbb{R}^{n+1}\right)}\right] .
\end{aligned}
$$

Using the Fourier transform we see that $A_{\delta}^{j, k} L_{j}\left(A_{\delta}^{j^{\prime} k^{\prime}} L_{j^{\prime}}\right)^{*}=0$ if either $\left|k-k^{\prime}\right|>4$ or $\left|j-j^{\prime}\right|>4$. This implies

$$
\left\|\sum_{j, k}\left(A_{\delta}^{j, k} L_{j}\right)^{*} h^{j, k}\right\|_{2} \leqslant C\left\|\left(\sum_{j, k}\left|h^{j, k}\right|^{2}\right)^{1 / 2}\right\|_{2}
$$

and, therefore, using duality

$$
\left(\sum_{j, k}\left\|A_{\delta}^{j, k} L_{j} g\right\|_{2}^{2}\right)^{1 / 2} \leqslant C\|g\|_{2} .
$$

Using (5.13) this and an analogous statement involving $\vartheta$-derivatives of $A_{\delta}^{j, k} L_{j} g$ imply that

$$
\left\|\sup _{\vartheta}\left|\sum_{k} B_{k} g(\cdot, \vartheta)\right|\right\|_{L^{2}\left(\mathbb{R}^{n}\right)} \leqslant C|\log \delta|^{1 / 2}\|g\|_{L^{2}\left(\mathbb{R}^{n+1}\right)} .
$$

By similar arguments we obtain

$$
\left\|\sup _{\vartheta}\left|\sum_{j} \tilde{A}_{\delta}^{j} g(\cdot, \vartheta)\right|\right\|_{L^{2}\left(\mathbb{R}^{n}\right)} \leqslant C|\log \delta|^{1 / 2}\|g\|_{L^{2}\left(\mathbb{R}^{n+1}\right)} .
$$

These two inequalities imply the statement of Theorem 5.1. 
Proof of (5.9) and (5.10). Let us notice that under our hypothesis the determinant of the $3 \times 3$ matrix

$$
\left(\begin{array}{c}
\nabla_{z} \varphi_{\eta}^{\prime}(z, \theta) \\
\nabla_{z}\left\langle\eta, \varphi_{\eta \eta}^{\prime \prime}(z, \theta) \theta^{\perp}\right\rangle
\end{array}\right)
$$

does not vanish if $\vartheta$ is close to 0 and $\eta /|\eta|$ is away from $(1,0)$. Here we have set $\theta(\vartheta)=(\cos \vartheta, \sin \vartheta)$ and $\theta^{\perp}(\vartheta)=(-\sin \vartheta, \cos \vartheta)$. This already implies that the operators $\widetilde{A}_{\delta}^{j}$ and $(\partial / \partial \vartheta) \widetilde{A}_{\delta}^{j}$ are Fourier integral operators associated to a local canonical graph; they are of order $-1 / 2$ and $1 / 2$, respectively. This implies (5.10), if $2^{j} \leqslant \delta^{-1}$. The analogous estimate for $2^{j}>\delta^{-1}$ follows from the same argument if one takes into account the decay properties of the symbol.

We now turn to the proof of (5.9), and again it suffices to consider the case $2^{j} \leqslant \delta^{-1}$. First observe that $\left(\partial / \partial_{\vartheta}\right)\left(\alpha_{\delta} \beta^{j, k}\right)=O\left(2^{j-k}\right)$. Since by homogeneity $\left\langle\varphi_{\eta \eta}^{\prime \prime}(z, \theta), \theta\right\rangle=0$, we see that on the supports of the symbols $(\partial / \partial \vartheta)\left\langle\varphi_{\eta}^{\prime}(z, \cos \vartheta, \sin \vartheta), \eta\right\rangle=O\left(2^{k}\right)$, and hence $(\partial / \partial \vartheta) A_{\delta}^{j, k}$ behaves like $2^{k} A_{\delta}^{j, k}$ if $k \geqslant j / 2$ and like $2^{j-k} A_{\delta}^{j, k}$ if $k \leqslant j / 2$. Therefore we only need to prove the $L^{2}$ estimate for $A_{\delta}^{j, k}$.

It turns out to be easier to prove the estimate for the adjoint operator because this allows us to use the Fourier transform. Specifically, if $\widetilde{f}$ denotes the partial Fourier transform of $f$ with respect to $y$, then the desired estimate is equivalent to

$$
\begin{aligned}
& \left(\int\left|\iint e^{i \phi(z, \eta, \vartheta)} \beta^{j, k}(\eta, \vartheta) \alpha_{\delta}(z, \eta, \vartheta) \tilde{f}(\eta, \vartheta) d \eta d \vartheta\right|^{2} d z\right)^{1 / 2} \\
& \leqslant C 2^{-k / 2} \min \left\{1,2^{k-j / 2}\right\}\|f\|_{L^{2}\left(\mathbb{R}^{3}\right)} .
\end{aligned}
$$

In order to prove it we distinguish two cases. The easy case arises if $k \leqslant j / 2$. In this case we change variables

$$
(\eta, \vartheta)=\left(2^{j} \xi, \vartheta(\xi)+\Theta\right)
$$

and dominate the left hand-side of $(5.15)$ by

$$
\begin{aligned}
& \int_{|\boldsymbol{\Theta}| \approx 2^{k-j}}\left(\int \mid \iint e^{i 2^{j} \phi(z, \xi, \vartheta(\xi)+\Theta)} \beta^{j, k}\left(2^{j} \xi, \vartheta(\xi)+\Theta\right)\right) \\
& \left.\times\left.\alpha_{\delta}\left(z, 2^{j} \xi, \vartheta(\xi)+\Theta\right) 2^{2 j} \widetilde{f}\left(2^{j} \xi, \vartheta(\xi)+\Theta\right) d \xi\right|^{2} d z\right)^{1 / 2} d \Theta .
\end{aligned}
$$

We split $z=(x, t)$ such that $\operatorname{det} \varphi_{\eta x}^{\prime \prime}(x, t, \theta) \neq 0$. Let for fixed $\Theta, t$

$$
\Phi(x, \xi ; \Theta, t)=\phi(x, t, \vartheta(\xi)+\Theta, \xi) .
$$

Then, if we set

$$
\theta^{\perp} \otimes \nabla_{\xi} \vartheta=\left(\begin{array}{cc}
-\sin \vartheta \frac{\partial \vartheta}{\partial \xi_{1}} & -\sin \vartheta \frac{\partial \vartheta}{\partial \xi_{2}} \\
\cos \vartheta \frac{\partial \vartheta}{\partial \xi_{1}} & \cos \vartheta \frac{\partial \vartheta}{\partial \xi_{2}}
\end{array}\right)
$$


it follows that

$$
\Phi_{\xi x}^{\prime \prime}(x, \xi ; \Theta, t)=\nabla_{x} \varphi_{\eta}^{\prime}+\left.\nabla_{x}\left(\xi^{t} \varphi_{\eta \eta}^{\prime \prime}\left(\theta^{\perp} \otimes \nabla_{\xi} \vartheta\right)\right)\right|_{(x, t, \theta)=(x, t, \theta(\vartheta(\xi)+\Theta))} .
$$

Observe that

$$
\left.\nabla_{x}\left(\xi^{t} \varphi_{\eta \eta}^{\prime \prime}\left(\theta^{\perp} \otimes \nabla_{\xi} \vartheta\right)\right)\right|_{\xi=\theta=(1,0)}=0,
$$

and we can conclude that the determinant of $\Phi_{\xi_{x}}^{\prime \prime}$ is bounded away from zero, for $\xi /|\xi|$ close to $(1,0)$ and $\vartheta$ close to 0 . Therefore we may apply the standard $L^{2}$ estimate for oscillatory integrals [21] and see that

$$
\begin{aligned}
& \left\|\left(A_{\delta}^{j, k}\right)^{*} f\right\|_{L^{2}\left(\mathbb{R}^{3}\right)} \\
& \quad \leqslant C 2^{-j} \int_{|\Theta| \approx 2^{k-j}}\left(\int\left|2^{2 j} \widetilde{f}\left(2^{j} \xi, \vartheta(\xi)+\Theta\right)\right|^{2} d \xi\right)^{1 / 2} d \Theta \\
& \quad \leqslant C 2^{(k-j) / 2} 2^{-j}\left\|2^{2 j} \widetilde{f}\left(2^{j} \cdot, \cdot\right)\right\|_{L^{2}\left(\mathbb{R}^{3}\right)}=C^{\prime} 2^{(k-j) / 2}\|f\|_{L^{2}\left(\mathbb{R}^{3}\right)}
\end{aligned}
$$

In order to prove $(5.15)$ for $k \geqslant j / 2$ we use a modification of the argument in Hörmander's proof of the Carleson-Sjölin lemma in [21]. Let $\Theta$ be as in (5.16) and make another change of variables, particularly,

$$
\begin{array}{cl}
\Theta(u)=|u|^{1 / 2}, & \text { if } u \geqslant 0, \\
\Theta(u)=-|u|^{1 / 2}, & \text { if } u \leqslant 0 .
\end{array}
$$

We set

$$
\Psi(z, \xi, u)=\phi(z, \xi, \vartheta(\xi)+\Theta(u))
$$

and

$$
T_{\delta}^{j} h(z)=\int e^{i 2^{j} \Psi(z, \xi, u)} \alpha_{\delta}(z, \xi, \vartheta(\xi)+\Theta(u)) h(\xi, u) d \xi d u
$$

Then

where

$$
\left(A_{\delta}^{j, k}\right)^{*} f=T_{\delta}^{j} h^{j, k}
$$

$$
h^{j, k}(\xi, u)=2^{2 j-1}|u|^{-1 / 2} \beta^{j, k}\left(2^{j} \xi, \vartheta(\xi)+\Theta(u)\right) \tilde{f}\left(2^{j} \xi, \vartheta(\xi)+\Theta(u)\right) .
$$

Since for $k \geqslant 1$

$$
\left\|h^{j, k}\right\|_{2} \leqslant C 2^{3 j / 2} 2^{-k / 2}\|f\|_{2}
$$

the desired estimate follows from

$$
\left\|T_{\delta}^{j} h\right\|_{2} \leqslant C 2^{-3 j / 2}\|h\|_{2}
$$

The square of the left-hand side of (5.21) equals

$$
\int_{\mathbb{R}^{3}} \int_{\mathbb{R}^{3}} H^{j}\left(\xi, u, \xi^{\prime}, u^{\prime}\right) h(\xi, u) \overline{h\left(\xi^{\prime}, u^{\prime}\right)} d \xi d u d \xi^{\prime} d u^{\prime}
$$

where

$$
\begin{aligned}
H^{j}\left(\xi, \dot{u}, \xi^{\prime}, u^{\prime}\right)=\int & e^{i 2^{j}\left(\Psi(z, \xi, u)-\Psi\left(z, \xi^{\prime}, u^{\prime}\right)\right)} \\
& \quad \times \alpha_{\delta}\left(z, 2^{j} \xi, \vartheta(\xi, u) \overline{\alpha_{\delta}\left(z, 2^{j} \xi^{\prime}, \vartheta\left(\xi^{\prime}, u^{\prime}\right)\right)} d z .\right.
\end{aligned}
$$


Now (5.21) of course follows if

$$
\left|H^{j}\left(\xi, u, \xi^{\prime}, u^{\prime}\right)\right| \leqslant C_{N}\left(1+2^{j}\left|\xi-\xi^{\prime}\right|+2^{j}\left|u-u^{\prime}\right|\right)^{-N}
$$

Recall that $\alpha_{\delta}$ is a $C^{\infty}$ function with respect to $z$. In order to perform the integration by parts necessary to prove $(5.24)$ we have to check that

$$
\left|\nabla_{z}\left(\Psi(z, \xi, u)-\Psi\left(z, \xi^{\prime}, u^{\prime}\right)\right)\right| \geqslant c_{0}\left(\left|\xi-\xi^{\prime}\right|+\left|u-u^{\prime}\right|\right)
$$

and

$$
\left|\partial_{z}^{\gamma}\left(\Psi(z, \xi, u)-\Psi\left(z, \xi^{\prime}, u^{\prime}\right)\right)\right| \leqslant C_{\gamma}\left(\left|\xi-\xi^{\prime}\right|+\left|u-u^{\prime}\right|\right) .
$$

Since for all $\gamma$

$$
\left.\partial_{\vartheta} \partial_{z}^{\gamma}\left\langle\varphi_{\eta}^{\prime}(z, \cos \vartheta, \sin \vartheta), \eta\right\rangle\right|_{\vartheta=\vartheta(\eta)}=0
$$

we see that $\Psi(z, \xi, \cdot)$ and all its derivatives with respect to $z$ are $C^{1}$ functions of $u$. This implies (5.26). Now if we could also show that $\operatorname{det} \Psi_{(\xi, u), z}^{\prime \prime} \neq 0$ then (5.25) would follow after possibly a further shrinking of the support of the symbols. Now

$$
\Psi_{(\xi, u), z}^{\prime \prime}(z, \xi, u)=\left.\left(\begin{array}{c}
\nabla_{z} \varphi_{\eta}^{\prime}+\nabla_{z}\left(\xi^{t} \varphi_{\eta \eta}^{\prime \prime}\left(\theta^{\perp} \otimes \nabla_{\xi} \vartheta\right)\right) \\
\nabla_{z}\left(\xi^{t} \varphi_{\eta \eta}^{\prime \prime} \theta^{\perp}\right)|u|^{-1 / 2} / 2
\end{array}\right)\right|_{\theta=\theta(\vartheta(\xi)+\Theta(u))},
$$

For $\vartheta$ close to 0 we have

$$
\left|\operatorname{det} \Psi_{z,(\xi, u)}^{\prime \prime}\right| \approx\left|\left\langle\theta^{\perp}(\vartheta+\Theta(u)), \xi\right\rangle\right||u|^{-1 / 2} \geqslant c_{0}^{\prime}
$$

for some positive constant $c_{0}^{\prime}$. This proves (5.25) and completes the proof of (5.9).

Proof of Theorem 5.2. The proof follows the same lines as the proof of Theorem 5.1. For $w \in \mathbb{R}^{n-1}$ near the origin let $\theta(w)=\left(w_{1}, \ldots, w_{n-1}, \sqrt{1-|w|^{2}}\right)$. Then one sets

$$
A_{\delta}^{j} g(y, w)=\int_{\mathbb{R}^{n+1}} \int_{\mathbb{R}^{n}} e^{i\left[\left\langle\varphi_{\eta}^{\prime}(z, \theta(w)), \eta\right\rangle-\langle y, \eta\rangle\right]} \alpha_{\delta}(z, \eta, w) \beta\left(2^{-j} \eta\right) g(z) d \eta d z,
$$

where now $\alpha_{\delta}(z, w, \eta)=\alpha(z, w) a(\delta \eta)$ with $a$ being a Schwartz function $a$ satisfying $\hat{a} \geqslant 0$ and with $\alpha \in C_{0}^{\infty}$ supported near the origin. As in the proof of Theorem 5.1, we may assume that

$$
\alpha_{\delta}(z, \eta, w)=0 \text { if }|\theta(w)-\eta /| \eta|| \geqslant 3 / 2 .
$$

The maximal inequality would then follow if we could show that for suitable small $\varepsilon_{0}$ and $2^{j}>\varepsilon_{0}^{-1}$

$$
\left\|\sup _{w}\left|A_{\delta}^{j} g(y, w)\right|\right\|_{L^{2}\left(\mathbb{R}^{n}\right)} \leqslant C_{N} 2^{j(n-2) / 2} \min \left\{1,\left(2^{j} \delta^{-1}\right)^{-N}\right\}\|g\|_{L^{2}\left(\mathbb{R}^{n+1}\right)} .
$$

We proceed as in the proof of Theorem 5.1 to decompose

$$
A_{\delta}^{j}=\widetilde{A}_{\delta}^{j}+\sum_{2^{k} \leqslant \varepsilon_{0} 2^{j}} A_{\delta}^{j, k} .
$$


In order to describe this decomposition define $w(\eta)$ by $\eta /|\eta|=\theta(w(\eta))$ and let

$$
\beta^{j, k}(\eta, w)=\beta\left(2^{k-j}|w-w(\eta)|\right) \beta\left(2^{-j} \eta\right) .
$$

For later use let us observe that if $\theta^{\perp}(w)$ is the hyperplane perpendicular to $\theta(w)$ and $\Pi_{\theta^{\perp}(w)}$ denotes the projection onto that hyperplane then

$$
\left|\Pi_{\theta^{\perp}(w)} \eta\right| \approx 2^{k-j} \quad \text { if }(\eta, w) \in \operatorname{supp} \beta^{j, k}, 2 \leqslant 2^{k} \leqslant \varepsilon_{0} 2^{j}
$$

If $k=0$ we define $\beta^{j, 0}$ in the analogous way, such that $\beta^{j, 0}$ is supported where $\eta \approx 2^{j}$ and $|w-w(\eta)| \leqslant 2^{-j+1}$, and let

$$
\tilde{\beta}^{j}(\eta, w)=\beta\left(2^{-j} \eta\right)\left(1-\sum_{2^{k}<\varepsilon_{0} 2^{j}} \beta^{j, k}(\eta, w)\right) .
$$

As in (5.8) we define

$$
\begin{aligned}
A_{\delta}^{j, k} g(y, \vartheta) & =e^{i\left[\left\langle\varphi_{\eta}^{\prime}(z, \theta(w)), \eta\right\rangle-\langle y, \eta\rangle\right]} \beta^{j, k}(\eta, w) \alpha_{\delta}(z, \eta, w) g(z) d \eta d z, \\
\tilde{A}_{\delta}^{j} g(y, w) & =\iint e^{i\left[\left\langle\varphi_{\eta}^{\prime}(z, \theta(w)), \eta\right\rangle-\langle y, \eta\rangle\right]} \tilde{\beta}^{j}(\eta, w) \alpha_{\delta}(z, \eta, w) g(z) d \eta d z .
\end{aligned}
$$

The desired inequality will clearly follow from

$$
\left\|\sup _{w}\left|A_{\delta}^{j, k} g(y, \theta)\right|\right\|_{2} \leqslant C_{N} 2^{(2 k-j)(n-2) / 2} \min \left\{1,\left(2^{-j} \delta^{-1}\right)^{-N}\right\}\|g\|_{2}
$$

$$
\begin{gathered}
\left\|\sup _{w}\left|A_{\delta}^{j, k} g(y, \theta)\right|\right\|_{2} \leqslant C_{N} \min \left\{1,\left(2^{-j} \delta^{-1}\right)^{-N}\right\}\|g\|_{2} \quad \text { if } k \leqslant j / 2, \\
\left\|\sup _{w}\left|\widetilde{A}_{\delta}^{j} g(y, \theta)\right|\right\|_{2} \leqslant C_{N} 2^{j(n-2) / 2} \min \left\{1,\left(2^{-j} \delta^{-1}\right)^{-N}\right\}\|g\|_{2} .
\end{gathered}
$$

We want to use the following higher-dimensional version of (5.11):

$$
\sup _{w \in \mathbb{R}^{n-1}}\left|F\left(w_{1}, \ldots, w_{n-1}\right)\right| \leqslant C \sum_{\gamma+\gamma^{\prime}=(1, \ldots, 1)}\left\|\partial_{w}^{\gamma} F\right\|_{2}^{1 / 2}\left\|\partial_{w}^{\gamma^{\prime}} F\right\|_{2}^{1 / 2}
$$

which is a straightforward consequence of

$$
\begin{aligned}
& F^{2}\left(w_{1}, \ldots, w_{n-1}\right) \\
& \quad=\int_{-\infty}^{w_{n-1}} \cdots \int_{-\infty}^{w_{1}} \frac{\partial}{\partial s_{n-1}} \cdots \frac{\partial}{\partial s_{1}} F^{2}\left(s_{1}, \ldots, s_{n-1}\right) d s_{1} \cdots d s_{n-1} .
\end{aligned}
$$


Applying (5.32) we see that (5.31) is a consequence of

$$
\left(\iint\left|\partial_{w}^{\gamma} \tilde{A}_{\delta}^{j} g(y, \theta)\right|^{2} d \theta d y\right)^{1 / 2} \leqslant C_{N} 2^{-j(1-2 \gamma) / 2} \min \left\{1,\left(2^{-j} \delta^{-1}\right)^{-N}\right\}\|g\|_{2},
$$

$$
\begin{aligned}
& \left(\iint\left|\partial_{w}^{\gamma} A_{\delta}^{j, k} g(y, \theta)\right|^{2} d \theta d y\right)^{1 / 2} \\
& \leqslant C_{N} 2^{(k-j)(n-2) / 2} 2^{-k(1-2 \gamma) / 2} \min \left\{1,\left(2^{-j} \delta^{-1}\right)^{-N}\right\}\|g\|_{2} \quad \text { if } k \geqslant j / 2,
\end{aligned}
$$

$$
\begin{aligned}
& \left(\iint\left|\partial_{w}^{\gamma} A_{\delta}^{j, k} g(y, \theta)\right|^{2} d \theta d y\right)^{1 / 2} \\
& \quad \leqslant C_{N} 2^{-(j-k)(n-1-2 \gamma) / 2} \min \left\{1,\left(2^{-j} \delta^{-1}\right)^{-N}\right\}\|g\|_{2} \text { if } k \leqslant j / 2 .
\end{aligned}
$$

Now $\partial_{w}^{\gamma} \widetilde{A}_{\delta}^{j}$ has the same quantitative properties as $2^{j|\gamma|} \widetilde{A}_{\delta}^{j}$, while $\partial_{w}^{\gamma} A_{\delta}^{j, k}$ behaves like $2^{k|\gamma|} A_{\delta}^{j, k}$ if $k \geqslant j / 2$ and like $2^{(j-k)|\gamma|} A_{\delta}^{j, k}$ if $k \leqslant j / 2$. Therefore we only need to prove the $L^{2}$ estimates for $|\gamma|=0$. The estimate for $\widetilde{A}_{\delta}^{j}$ is immediate if one observes that

$$
\operatorname{rank}\left(\begin{array}{c}
\nabla_{z} \varphi_{\eta}^{\prime}(z, \theta) \\
\nabla_{z}\left(\xi^{t} \varphi_{\eta \eta}^{\prime \prime}(z, \theta) \frac{d \theta}{d w}\right)
\end{array}\right)=n+1
$$

for $\theta$ close to $(0, \ldots, 0,1)$ and $\xi /|\xi|$ away from $(0, \ldots, 0,1)$.

Instead of estimating $A_{\delta}^{j, k}$ we shall estimate its adjoint using the Fourier transform. Also we shall only consider the case $2^{j} \leqslant \delta^{-1}$ since the remaining cases follow by the same arguments, taking into account the rapid decay of the symbol. We change variables

$$
(\eta, w)=\left(2^{j} \xi, w(\xi)+\omega\right)
$$

and let

$$
\begin{aligned}
T_{\delta}^{j, k} h(z)=\iint & e^{i 2^{j} \Phi(z, \xi, \omega)} \beta^{j, k}(\xi, w(\xi)+\omega) \\
& \times \alpha_{\delta}\left(z, 2^{j} \xi, w(\xi)+\omega\right) h(\xi, \omega) d \xi d \omega
\end{aligned}
$$

where

$$
\Phi(z, \xi, \omega)=\left\langle\varphi_{\eta}^{\prime}(z, \theta(w(\xi)+\omega)), \xi\right\rangle
$$

Then

$$
\left(A_{\delta}^{j, k} f\right)^{*}(z)=2^{j n} T_{\delta}^{j, k} h^{j}
$$

where

$$
h^{j}(\xi, \omega)=\tilde{f}\left(2^{j} \xi, w(\xi)+\omega\right),
$$

and it suffices to prove

$$
\left\|T_{\delta}^{j, k} h\right\|_{2} \leqslant C 2^{-(j-k)(n-2) / 2} 2^{-(j n+k) / 2} \min \left\{1,2^{k-j / 2}\right\}\|h\|_{2} .
$$


The case $k \leqslant j / 2$ is handled exactly in the same manner as the two-dimensional case. One uses the fact that $\operatorname{rank} \Phi_{\xi z}^{\prime \prime} \geqslant n$ and that for fixed $\xi$ the function $\omega \rightarrow \beta^{j, k}(\xi, w(\xi)+\omega)$ is supported on a set of measure $\leqslant C 2^{-(j-k)(n-1)}$.

Next, let us assume that $2^{j / 2} \leqslant 2^{k} \leqslant \varepsilon_{0} 2^{j}$. In this case we need to introduce a further finite decomposition of the operator $T_{\delta}^{j, k}$. First note that the nondegeneracy and cone conditions imply that for any unit vector $e$ that is orthogonal to $\theta(w(\xi)+\omega)$

$\operatorname{rank} \Phi_{(\xi, \omega), z}^{\prime \prime}(z, e)=\left.\operatorname{rank}\left(\begin{array}{c}\nabla_{z} \varphi_{\eta}^{\prime}+\nabla_{z}\left(e^{t} \varphi_{\eta \eta}^{\prime \prime} \frac{d \theta}{d w} \frac{d w}{d \xi}\right) \\ \nabla_{z}\left(e^{t} \varphi_{\eta \eta}^{\prime \prime} \frac{d \theta}{d w}\right)\end{array}\right)\right|_{w=w(e)+\omega} \equiv n+1$.

Therefore, one can find for each $\theta$ a finite partition of unity of $\theta^{\perp}$ consisting of functions $\chi_{l}(\cdot, \theta), 1 \leqslant l \leqslant M$, smoothly depending on $\theta$, such that each $\chi_{l}(\cdot, \theta) \in C^{\infty}\left(\theta^{\perp} \backslash 0\right)$ is homogeneous of degree 0 and such that for $1 \leqslant l \leqslant M$ there is $i(l)$ with

$$
\left.\operatorname{det}\left(\begin{array}{c}
\nabla_{z} \varphi_{\eta}^{\prime}+\nabla_{z}\left(e^{t} \varphi_{\eta \eta}^{\prime \prime} \frac{d \theta}{d w} \frac{d w}{d \xi}\right) \\
\nabla_{z}\left(e^{t} \varphi_{\eta \eta}^{\prime \prime} \frac{d \theta}{d w_{i(l)}}\right)
\end{array}\right)\right|_{(z, \theta)=(z, \theta(w(\xi)+\omega))} \geqslant c_{0}>0,
$$

if $e$ is a unit vector in $\theta^{\perp}$. Moreover, if $\Pi_{\xi, \omega}^{\perp}$ denotes the projection onto the hyperplane perpendicular to $\theta(w(\xi)+\omega)$ and if $\xi$ is close to $(0, \ldots, 0,1)$ then

$$
\begin{aligned}
\left.\operatorname{det}\left(\begin{array}{c}
\nabla_{z} \varphi_{\eta}^{\prime}+\nabla_{z}\left(\xi^{t} \varphi_{\eta \eta}^{\prime \prime} \frac{d \theta}{d w} \frac{d w}{d \xi}\right) \\
\left|\Pi_{\xi, \omega}^{\perp} \xi\right|^{-1} \nabla_{z}\left(\xi^{t} \varphi_{\eta \eta}^{\prime \prime} \frac{d \theta}{d w_{i(l)}}\right)
\end{array}\right)\right|_{(z, \theta)=(z, \theta(w(\xi)+\omega))} \geqslant c_{0}>0, \\
\quad \text { if } \chi_{l}\left(\Pi_{\xi, \omega}^{\perp} \xi, \theta(w(\xi)+\omega)\right) \neq 0 .
\end{aligned}
$$

Also we see that the matrix in (5.39) and its inverse have uniformly bounded matrix norms, provided $\xi, \theta$ are close to $(0, \ldots, 0,1)$. Let

$\beta_{\delta, l}^{j, k}(z, \xi, \omega)=\alpha_{\delta}(z, \xi, w(\xi)+\omega) \beta^{j, k}(\xi, w(\xi)+\omega) \chi_{l}\left(\Pi_{\xi, \omega}^{\perp} \xi, \theta(w(\xi)+\omega)\right)$ and define

$$
T_{\delta, l}^{j, k} h(z)=\iint e^{i 2^{j} \Phi(z, \xi, \omega)} \beta_{\delta, l}^{j, k}(z, \xi, \omega) h(\xi, \omega) d \xi d \omega .
$$

We show that for $j / 2 \leqslant k$ the operators $T_{\delta, l}^{j, k}$ satisfy the bounds (5.38) and fix $l$. For notational reasons we may assume that the distinguished variable $\omega_{i(l)}$ in (5.39) is $\omega_{1}$. We may also assume that for $\omega, \omega^{\prime} \in \operatorname{supp} \beta_{\delta, l}^{j, k}(z, \xi, \cdot)$ one has $\left|\omega^{2}-\left(\omega^{\prime}\right)^{2}\right| \leqslant \varepsilon 2^{k-j}\left|\omega-\omega^{\prime}\right|$, for a small constant $\varepsilon>0$. We split $\omega=\left(\omega_{1}, v\right)$ and write

$$
T_{\delta, l}^{j, k} h(z)=\int_{v} T_{\delta, l}^{j, k, v} h_{v}(z) d v
$$


where $h_{v}\left(\xi, \omega_{1}\right)=h(\xi, \omega)$ and

$$
T_{\delta, l}^{j, k, v} h_{v}(z)=\iint e^{i 2^{j} \Phi\left(z, \xi, \omega_{1}, v\right)} \beta_{\delta, l}^{j, k}\left(z, \xi, \omega_{1}, v\right) h_{v}\left(\xi, \omega_{1}\right) d \xi d \omega_{1} .
$$

Since the $v$ integration in (5.40) is extended over a set of measure $\leqslant$ $C 2^{-(j-k)(n-2)}$, the desired inequality follows from

$$
\left\|T_{\delta, l}^{j, k, v} h_{v}\right\|_{2} \leqslant C 2^{-(j n+k) / 2}\left\|h_{v}\right\|_{2},
$$

uniformly in $v$. In order to show this we estimate the kernel of $\left(T_{\delta, l}^{j, k, v}\right)^{*} T_{\delta, l}^{j, k, v}$, which is

$$
\begin{aligned}
\mathscr{K}_{\delta, l}^{j, k, v}\left(\xi, \omega_{1}, \xi^{\prime}, \omega_{1}^{\prime}\right)=\int e^{i 2^{j}\left[\Phi\left(z, \xi, \omega_{1}, v\right)-\Phi\left(z, \xi^{\prime}, \omega_{1}^{\prime}, v\right)\right]} \\
\quad \times \beta_{\delta, l}^{j, k}\left(z, \xi, \omega_{1}, v\right) \overline{\beta_{\delta, l}^{j, k}\left(z, \xi^{\prime}, \omega_{1}^{\prime}, v\right)} d z .
\end{aligned}
$$

Then (5.41) would follow from

$$
\left|\mathscr{K}_{\delta, l}^{j, k, v}\left(\xi, \omega_{1}, \xi^{\prime}, \omega_{1}^{\prime}\right)\right| \leqslant C_{N}\left(1+2^{j}\left|\xi-\xi^{\prime}\right|+2^{k}\left|\omega_{1}-\omega_{1}^{\prime}\right|\right)^{-N} .
$$

For $(\xi, \omega) \in \operatorname{supp} \beta_{\delta, l}^{j, k}$ we have $\left|\Pi_{\xi, \omega}^{\perp} \xi\right| \approx 2^{k-j}$, and this implies using Taylor's formula

$$
\begin{aligned}
& \left|\partial_{z}^{\gamma}\left[\Phi\left(z, \xi, \omega_{1}, v\right)-\Phi\left(z, \xi^{\prime}, \omega_{1}^{\prime}, v\right)\right]\right| \\
& \quad \leqslant C_{\gamma}\left(\left|\xi-\xi^{\prime}\right|+2^{k-j}\left|\omega_{1}-\omega_{1}^{\prime}\right|\right)+C_{\gamma}\left(\left|\xi-\xi^{\prime}\right|^{2}+\left|\omega_{1}-\omega_{1}^{\prime}\right|^{2}\right) \\
& \quad \leqslant C_{\gamma}^{\prime}\left(\left|\xi-\xi^{\prime}\right|+2^{k-j}\left|\omega_{1}-\omega_{1}^{\prime}\right|\right) .
\end{aligned}
$$

For the last inequality we used the assumption on the support of the symbols $\beta_{\delta, l}^{j, k}$. On the other hand (5.39) implies that

$$
\begin{aligned}
& \left|\nabla_{z}\left[\Phi\left(z, \xi, \omega_{1}, v\right)-\Phi\left(z, \xi^{\prime}, \omega_{1}^{\prime}, v\right)\right]\right| \\
& \quad \geqslant c\left(\left|\xi-\xi^{\prime}\right|+2^{k-j}\left|\omega_{1}-\omega_{1}^{\prime}\right|\right)-C\left(\left|\xi-\xi^{\prime}\right|^{2}+\left|\omega_{1}-\omega_{1}^{\prime}\right|^{2}\right) \\
& \quad \geqslant c^{\prime}\left(\left|\xi-\xi^{\prime}\right|+2^{k-j}\left|\omega_{1}-\omega_{1}^{\prime}\right|\right)
\end{aligned}
$$

using again the assumptions on the support. Now using (5.43) and (5.44) the estimate $(5.42)$ follows immediately by integration by parts.

Finally let us state an $L^{2}$ estimate for a nonisotropic variant of the Kakeya maximal function. For simplicity we do not formulate an invariant statement and consider only canonical relations $\mathscr{C}$ as in (2.7). For $\varphi$ as in (2.7), $\chi \in$ $C_{0}^{\infty}\left(\mathbb{R}^{n} \times \mathbb{R}^{n+1}\right)$, and $\theta \in S^{n-1}$ let

$$
\begin{aligned}
H_{\delta_{1}, \delta_{2}}^{\theta}(y, z)= & \chi(y, z) \frac{\delta_{1}^{-1}}{\left(1+\delta_{1}^{-1}\left|\left\langle\varphi_{\eta}^{\prime}(z, \theta)-y, \theta\right\rangle\right|\right)^{N}} \\
& \times \frac{\delta_{2}^{-(n-1)}}{\left(1+\delta_{2}^{-1}\left|\Pi_{\theta^{\perp}}\left(\varphi_{\eta}^{\prime}(z, \theta)-y\right)\right|\right)^{N}}
\end{aligned}
$$


where $0<\delta_{1} \leqslant \delta_{2} \leqslant 1 / 2, N>n+1$, and $\Pi_{\theta^{\perp}}$ denotes the orthogonal projection onto the hyperplane orthogonal to $\theta$. Let

$$
\mathscr{M}_{\delta_{1}, \delta_{2}} g(y)=\sup _{\theta \in S^{n-1}} \int\left|H_{\delta_{1}, \delta_{2}}^{\theta}(y, z) g(z)\right| d z .
$$

Then we have

Theorem 5.3. Let $\delta_{1} \leqslant \delta_{2} \leqslant 1 / 2$ and $\mathscr{M}_{\delta_{1}, \delta_{2}}$ as in (5.45). Then, if $n=2$,

and, if $n \geqslant 3$,

$$
\left\|\mathscr{M}_{\delta_{1}, \delta_{2}} g\right\|_{L^{2}\left(\mathbb{R}^{2}\right)} \leqslant C\left|\log \delta_{1}\right|^{1 / 2}\|g\|_{L^{2}\left(\mathbb{R}^{3}\right)}
$$

$$
\left\|\mathscr{M}_{\delta_{1}, \delta_{2}} g\right\|_{L^{2}\left(\mathbb{R}^{n}\right)} \leqslant C \delta_{1}^{-(n-2) / 2}\|g\|_{L^{2}\left(\mathbb{R}^{n+1}\right)} .
$$

Proof. We define the nonisotropic averaging operators

$$
A_{\delta_{1}, \delta_{2}} g(y, \theta)=\int_{\mathbb{R}^{n+1}} \int_{\mathbb{R}^{n}} e^{i\left\langle\varphi_{\eta}^{\prime}(z, \theta)-y, \eta\right\rangle} \alpha(z, \theta) a_{\delta_{1}, \delta_{2}}(\eta, \theta) d \eta g(z) d z .
$$

Here $\alpha \in C_{0}^{\infty}$ with small support and

$$
a_{\delta_{1}, \delta_{2}}(\eta, \theta)=a_{1}\left(\delta_{1}^{-1}\langle\eta, \theta\rangle\right) a_{2}\left(\delta_{2}^{-1} \Pi_{\theta^{\perp}} \eta\right)
$$

for suitable Schwartz functions $a_{1}, a_{2}$ in $\mathbb{R}$, and $\mathbb{R}^{n-1}$, respectively. Using rescaling arguments we may assume that $a_{1}, a_{2}$ have compact support. Then it suffices to prove the required estimates for the maximal function $\sup _{\theta}\left|A_{\delta_{1}, \delta_{2}} g(\cdot, \theta)\right|$. This is done in exactly the same way as in the isotropic case. The only difference concerns the smoothness in the $\theta$ variable of the symbol $a_{\delta_{1}, \delta_{2}}$; namely, we have $\partial_{\theta}^{\gamma} a_{\delta_{1}, \delta_{2}}=O\left(\left|\eta \delta_{2}\right|^{-|\gamma|}\right)$. Note, however, that if $\tilde{\beta}^{j}, \beta^{j, k}$ are as in the proof of Theorems 5.1 and 5.2 then $a_{\delta_{1}, \delta_{2}} \beta^{j, k}=0$ if $2^{k} \geqslant C \delta_{2}^{-1}$ and $a_{\delta_{1}, \delta_{2}} \tilde{\beta}^{j}=0$ if $\delta_{2}^{-1}$ is small compared to $2^{j}$. Therefore, the $\theta$ derivatives of $a_{\delta_{1}, \delta_{2}} \beta^{j, k}$ and $a_{\delta_{1}, \delta_{2}} \tilde{\beta}^{j}$ behave like the ones of $a_{\delta_{1}} \beta^{j, k}$ and $a_{\delta_{1}} \tilde{\beta}^{j}$, respectively (where $a_{\delta_{1}}$ is as in the isotropic case). This is enough to carry over the previous arguments.

\section{6. $L^{p} \rightarrow L^{p}$ LOCAL SMOOTHING IN TWO DIMENSIONS AND CARLESON-SJÖLIN ESTIMATES}

In this section we shall extend the $L^{p}$ local smoothing results of $\S 4$ to two dimensions and prove a sharp square function estimate for Fourier integral operators which will be the basic prerequisite for the multiplier estimates in $\S 7$. In the following we shall always assume that $X, Y, Z$ be paracompact manifolds with $\operatorname{dim} X=\operatorname{dim} Y=2, \operatorname{dim} Z=3 ; I$ will be a compact subinterval of $\mathbb{R}$.

Theorem 6.1. Suppose that $\mathscr{F} \in I^{\mu-1 / 4}(Z, Y ; \mathscr{C})$ where $\mathscr{C}$ satisfies the nondegeneracy assumption (2.2), (2.3) and the cone condition (2.6). Then $\mathscr{F}$ is bounded from $L_{\mathrm{loc}}^{p}(Y)$ to $L_{\mathrm{comp}}^{p}(Z)$ if $\mu \leqslant-(1 / 2-1 / p)+\varepsilon(p)$, where

$$
\varepsilon(p)= \begin{cases}\frac{1}{2 p}, & 4 \leqslant p<\infty, \\ \frac{1}{2}\left(\frac{1}{2}-\frac{1}{p}\right), & 2<p \leqslant 4 .\end{cases}
$$


Our next result is a sharp mixed-norm $L^{p} \rightarrow L^{q}\left(L^{2}\right)$ estimate, for $2<p \leqslant 4$. It is in the spirit of Theorem 3.2, which covers the case $p=2$ and $q \geqslant 6$.

Theorem 6.2. Suppose that $\mathscr{F} \in I^{\mu-1 / 4}(X \times \mathbb{R}, Y ; \mathscr{C})$, where $\mathscr{C} \subset T^{*} X \backslash 0 \times$ $T^{*} \mathbb{R} \backslash 0 \times T^{*} Y \backslash 0$ satisfies the nondegeneracy assumptions (2.2), (2.3) and the cone condition (2.6). Let

$$
G(f)(x)=\left(\int_{I}|\mathscr{F} f(x, t)|^{2} d t\right)^{1 / 2} .
$$

Then if $2 \leqslant p \leqslant 4, q=3 p /(p-1)$, and $\mu<\mu(q)=-2(1 / 2-1 / q)+1 / 2$ it follows that $G$ is bounded from $L_{\text {comp }}^{p}(Y)$ to $L_{\text {loc }}^{q}(X)$.

If $p=4$ we have also $q=4$ and, in the Euclidean case, Theorem 6.2 is essentially equivalent to Carbery's square function estimate for Bochner-Riesz multipliers [7, 23].

As in $\S 4$ we may state two straightforward consequences of Theorem 6.1 concerning maximal operators and regularity of solutions of the wave equation. They improve results in $[4,32]$, where weaker estimates were proved under an additional conormality assumption. In the Euclidean case the analogous results have been obtained in [24].

Corollary 6.3. Assume that $X, Y$, and $I$ are compact and $\mathscr{F} \in$ $I^{\mu-1 / 4}(X \times I, Y ; \mathscr{C})$ where $\mathscr{C}$ is as in Theorem 6.2. Then

$$
\left\|\sup _{t \in I}|\mathscr{F} f(\cdot, t)|\right\|_{L^{p}(X)} \leqslant C\|f\|_{L^{p}(Y)},
$$

if $\mu<-1 / 2-\varepsilon(p)$, where $\varepsilon(p)$ is given by (6.1).

Corollary 6.4. Let $u$ be the solution to the Cauchy problem (3.2). Then, if $I \subset \mathbb{R}$ is a compact interval and if $\varepsilon<\varepsilon(p), \beta_{p}=|1 / 2-1 / p|$,

$$
\|u\|_{L_{\alpha-\beta_{p}+\varepsilon}^{p}(M \times I)} \leqslant C\left(\|f\|_{L_{\alpha}^{p}(M)}+\|g\|_{L_{\alpha}^{p}(M)}\right), \quad 2<p<\infty .
$$

In the proofs of Theorems 6.1 and 6.2 we use an adaptation of the arguments in [24]. As in the proof of Theorem 4.1 one tries to use orthogonality arguments, which exploits the cone condition to replace the operator $\mathscr{F}$ by certain "square functions" involving an angular decomposition of the operators $\mathscr{F}$. These square functions can then be handled by using our estimates on variable coefficient Kakeya maximal functions in $\S 5$.

Proof of Theorem 6.1. As in the proof of Theorem 4.1 we may assume that $\mathscr{F}=\mathscr{F}$ is of the form (4.5) and that its symbol is of order zero and supported in $\left\{\xi:\left(1-a_{0}\right) \lambda \leqslant|\xi| \leqslant\left(1+a_{0}\right) \lambda\right\}$, for a small constant $a_{0}$. We also assume that the symbol vanishes for $x \in \mathbb{R}^{2}$ and $t \in \mathbb{R}$ outside of a fixed compact set and that the $\eta$ support of the symbol is contained in a narrow cone in which the map $\eta \rightarrow \varphi_{x}^{\prime}(x, t, \eta)$ is an isomorphism.

The statement of the theorem follows from

$$
\left\|\mathscr{F}_{\lambda} f\right\|_{L^{4}\left(\mathbb{R}^{3}\right)} \leqslant C_{\varepsilon} \lambda^{1 / 8+\varepsilon}\|f\|_{L^{4}\left(\mathbb{R}^{2}\right)}, \quad \lambda>1, \varepsilon>0 .
$$


As in $\S 4$ we make an angular decomposition $\mathscr{F}_{\lambda}=\sum_{\nu} \mathscr{F}_{\lambda}^{\nu}$ with

$$
\mathscr{F}_{\lambda}^{\nu} f(x, t)=\int e^{i \varphi(x, t, \eta)} \chi_{\nu}(\eta) a_{\lambda}(x, t, \eta) \hat{f}(\eta) d \eta
$$

Here $\chi_{\nu}$ is supported in a sector of angle $\approx \lambda^{-1 / 2}$ that contains the unit vector $\eta_{\nu}$. We shall also assume that the indices $\nu$ are chosen so that, if we identify $\mathbb{R}^{2}$ and $\mathbb{C}$ in the obvious way, then $\arg \eta_{\nu}<\arg \eta_{\nu+1}$, and therefore $\left|\eta_{\nu}-\eta_{\nu+1}\right| \approx \lambda^{-1 / 2}$.

Then

$$
\left\|\mathscr{F}_{\lambda} f\right\|_{L^{4}\left(\mathbb{R}^{3}\right)} \leqslant 2 \sum_{\ell}\left\|\sum_{\substack{|\nu-\mu| \approx 2^{\ell} \\ \mu \leqslant \nu}} \mathscr{F}_{\lambda}^{\nu} f \mathscr{F}_{\lambda}^{\mu} f\right\|_{L^{2}\left(\mathbb{R}^{3}\right)} .
$$

Since we are assuming that $a$ has small conic support, the summation only involves nonnegative indices $\ell$ with $2^{\ell}<\delta \lambda^{1 / 2}$, where $\delta>0$ is small.

We shall need to make a further decomposition based on $\ell$. To this end, we fix $\rho \in C_{0}^{\infty}((-1,1))$ as before satisfying $\sum_{j \in \mathbb{Z}}(\rho(u-j))^{2} \equiv 1$. We then set

$$
\mathscr{F}_{\lambda, \ell}^{\nu, j} f(x, t)=\int e^{i \varphi(x, t, \eta)} a_{\lambda, \ell}^{\nu, j}(x, t, \eta) \hat{f}(\eta) d \eta
$$

with

$$
a_{\lambda, \ell}^{\nu, j}(x, t, \eta)=\chi_{\nu}(\eta) \rho\left(\lambda^{-1} 2^{\ell} \varphi_{t}^{\prime}(x, t, \eta)-j\right) a_{\lambda}(x, t, \eta) .
$$

Finally at the end of the proof we shall need a similar but finer decomposition of the operators $\mathscr{F}_{\lambda, \ell}^{\nu, j}$, namely, we set

$$
\mathscr{F}_{\lambda, \ell}^{\nu, j, m} f(x, t)=\int e^{i \varphi(x, t, \eta)} a_{\lambda, \ell}^{\nu, j, m}(x, t, \eta) \hat{f}(\eta) d \eta,
$$

with

$$
a_{\lambda, \ell}^{\nu, j, m}(x, t, \eta)=\rho\left(\lambda^{-1 / 2} \varphi_{t}^{\prime}(x, t, \eta)-m\right) a_{\lambda, \ell}^{\nu, j}(x, t, \eta) .
$$

Notice that $\operatorname{supp} a_{\lambda, \ell}^{\nu, m} \subset \operatorname{supp} a_{\lambda, \ell}^{\nu, j}$. Furthermore, for fixed $x, t, \operatorname{supp} \eta \rightarrow$ $a_{\lambda, \ell}^{\nu, j}$ is comparable to a $\lambda 2^{-\ell} \times \lambda^{1 / 2}$ rectangle, while supp $\eta \rightarrow a_{\lambda, \ell}^{\nu, j, m}$ is comparable to a cube of sidelength $\lambda^{1 / 2}$.

We need two different orthogonality properties of the operators $\mathscr{F}_{\lambda, \ell}^{\nu, j}$. The first says that $\mathscr{F}_{\lambda, \ell}^{\nu, j} f \mathscr{F}_{\lambda, \ell}^{\mu, k} f$ and $\mathscr{F}_{\lambda, \ell}^{\nu, j^{\prime}} f \mathscr{F}_{\lambda, \ell}^{\mu, k^{\prime}} f$ are almost orthogonal if $j+k$ is different from $j^{\prime}+k^{\prime}$. This and a similar statement for the operators $\mathscr{F}_{\lambda, \ell}^{\nu, j, m}$ is contained in

Lemma 6.6. Suppose $\left|j+k-j^{\prime}-k^{\prime}\right| \geqslant \lambda^{\varepsilon}$. Then

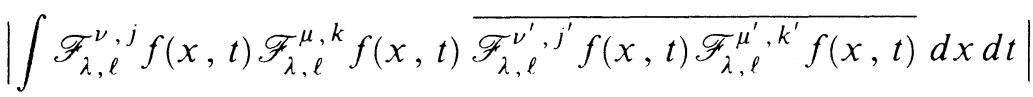

$$
\begin{aligned}
& \leqslant C_{\varepsilon N} \lambda^{-N}\|f\|_{4}^{4} \text {. }
\end{aligned}
$$


Suppose $\left|n+m-n^{\prime}-m^{\prime}\right| \geqslant \lambda^{\varepsilon}$. Then

$$
\begin{aligned}
& \left|\int \mathscr{F}_{\lambda, \ell}^{\nu, j, m} f(x, t) \mathscr{F}_{\lambda, \ell}^{\mu, k, n} f(x, t) \overline{\mathscr{F}_{\lambda, \ell}^{\nu^{\prime}, j^{\prime}, m^{\prime}} f(x, t) \mathscr{F}_{\lambda, \ell}^{\mu^{\prime}, k^{\prime}, n^{\prime}} f(x, t)} d x d t\right| \\
& \quad \leqslant C_{\varepsilon N} \lambda^{-N}\|f\|_{4}^{4} .
\end{aligned}
$$

The second orthogonality property says that

$$
\mathscr{F}_{\lambda, \ell}^{\nu, j} f \mathscr{F}_{\lambda, \ell}^{\mu, k} f \text { and } \mathscr{F}_{\lambda, \ell}^{\nu^{\prime}, j} f \mathscr{F}_{\lambda, \ell}^{\mu^{\prime}, k} f
$$

are almost orthogonal if the distance between the pairs $(\nu, \mu)$ and $\left(\nu^{\prime}, \mu^{\prime}\right)$ is large.

Lemma 6.7. Suppose that $\mu \leqslant \nu, \mu^{\prime} \leqslant \nu^{\prime}$, and $\left|\nu-\nu^{\prime}\right|+\left|\mu-\mu^{\prime}\right| \geqslant \lambda^{\varepsilon}$. Then

$$
\begin{aligned}
& \left|\int \mathscr{F}_{\lambda, \ell}^{\nu, j} f(x, t) \mathscr{F}_{\lambda, \ell}^{\mu, k} f(x, t) \overline{\mathscr{F}_{\lambda, \ell}^{\nu^{\prime}, j} f(x, t) \mathscr{F}_{\lambda, \ell}^{\mu^{\prime}, k} f(x, t)} d x d t\right| \\
& \quad \leqslant C_{N} \lambda^{-N}\|f\|_{4}^{4} .
\end{aligned}
$$

The proofs of both lemmas both rely on integration by parts arguments. The one needed for (6.6) and (6.7) is straightforward and only uses the assumption that $\varphi_{t}^{\prime}(x, t, \eta) \neq 0$ for $\eta \neq 0$. The one needed for $(6.8)$ is more subtle and relies on the observation that the mapping $(\eta, \xi) \rightarrow(\eta+\xi, q(\eta)+q(\xi))$ from $\mathbb{R}^{4}$ to $\mathbb{R}^{3}$ is a submersion if $\eta \neq \xi$, provided the cone condition rank $q_{\eta \eta}^{\prime \prime}=1$ is satisfied. In fact, if we consider the cones $\Gamma_{x, t}$ introduced in (2.4) and define subsets

$$
\Gamma_{x, t}^{\ell, \nu, j}=\left\{\left(\varphi_{x}^{\prime}(x, t, \eta), q\left(x, t, \varphi_{x}^{\prime}(x, t, \eta)\right)\right): \eta \in \operatorname{supp} a_{\lambda, \ell}^{\nu, j}\right\},
$$

then we need precise estimates for the distance between the algebraic sums $\Gamma_{x, t}^{\ell, \nu}+\Gamma_{x, t}^{\ell, \mu, k}$ and $\Gamma_{x, t}^{\ell, \nu^{\prime}, j}+\Gamma_{x, t}^{\ell, \mu^{\prime}, k}$. In the constant coefficient case these correspond to certain overlapping properties of those sums (see [12, 15, 24]); however, in the present (variable coefficient) context a more careful analysis is required. The details of these orthogonality arguments are carried out in a subsection below.

We now apply the orthogonality property (6.6). Since for a fixed positive integer $s \leqslant 2^{\ell+1}$ there are $2^{\ell+1}$ pairs $(j, k)$ of positive integers such that $j+k=s$, we can apply (6.6) together with the Cauchy-Schwarz inequality to obtain

$$
\begin{aligned}
\left\|\mathscr{F}_{\lambda} f\right\|_{L^{4}\left(\mathbb{R}^{3}\right)}^{2} \leqslant & C \lambda^{\varepsilon} \sum_{\ell}\left(\sum_{s \leqslant 2^{\ell+1}}\left\|\sum_{j+k=s} \sum_{|\nu-\mu| \approx 2^{\ell}} \mathscr{F}_{\lambda, \ell}^{\nu, j}(f) \mathscr{F}_{\lambda, \ell}^{\mu, k}(f)\right\|_{L^{2}\left(\mathbb{R}^{3}\right)}^{2}\right)^{1 / 2} \\
& +C_{\varepsilon, N} \lambda^{-N}\|f\|_{L^{4}\left(\mathbb{R}^{2}\right)}^{2} \\
\leqslant & C_{\varepsilon} \lambda^{\varepsilon} \sum_{\ell} 2^{\ell / 4}\left\|\left(\sum_{j, k}\left|\sum_{|\nu-\mu| \approx 2^{\ell}} \mathscr{F}_{\lambda, \ell}^{\nu, j}(f) \mathscr{F}_{\lambda, \ell}^{\mu, k}(f)\right|^{2}\right)^{1 / 2}\right\|_{L^{2}\left(\mathbb{R}^{3}\right)} \\
& +C_{\varepsilon, N} \lambda^{-N}\|f\|_{L^{4}\left(\mathbb{R}^{2}\right)}^{2} .
\end{aligned}
$$


Applying (6.8) and the Cauchy-Schwarz inequality one more time yields

$$
\begin{aligned}
& \left\|\left(\sum_{j, k}\left|\sum_{|\nu-\mu| \approx 2^{\ell}} \mathscr{F}_{\lambda, \ell}^{\nu, j}(f) \mathscr{F}_{\lambda, \ell}^{\mu, k}(f)\right|^{2}\right)^{1 / 2}\right\|_{L^{2}\left(\mathbb{R}^{3}\right)} \\
& \leqslant C_{\varepsilon} \lambda^{\varepsilon}\left\|\left(\sum_{\nu, j}\left|\mathscr{F}_{\lambda, \ell}^{\nu, j}(f)\right|^{2}\right)^{1 / 2}\right\|_{L^{4}\left(\mathbb{R}^{3}\right)}^{2}+C_{\varepsilon, N} \lambda^{-N}\|f\|_{L^{4}\left(\mathbb{R}^{2}\right)}^{2} .
\end{aligned}
$$

Remark. The latter arguments are similar to an orthogonality argument in [24], where one works with a vertical decomposition (in $\tau=q(\xi)$ ) of size $\lambda^{1 / 2}$. Such a decomposition would produce symbols of type $(1 / 2,1 / 2)$, and the geometric arguments which rely on the curvature condition needed in order to prove Lemma 6.7 would fail for $2^{\ell} \ll \lambda^{1 / 2}$. For that reason we have to work with a vertical decomposition of size $2^{-\ell} \lambda$.

Having applied Lemma 6.7 we can introduce the finer decomposition $\mathscr{F}_{\lambda, \ell}^{\nu, j}=$ $\sum \mathscr{F}_{\lambda, \ell}^{\nu, j, m}$ where the sum involves less than $C \lambda^{1 / 2} 2^{-\ell}$ terms. If we now use (6.7) for $\nu=\nu^{\prime}, \mu=\mu^{\prime}, j=j^{\prime}, k=k^{\prime}$ and repeat the argument that led to (6.9), we obtain

$$
\begin{aligned}
\left\|\left(\sum_{\nu, j}\left|\mathscr{F}_{\lambda, \ell}^{\nu, j}(f)\right|^{2}\right)^{1 / 2}\right\|_{L^{4}\left(\mathbb{R}^{3}\right)}^{2} \leqslant & C_{\varepsilon} \lambda^{\varepsilon}\left(\lambda^{1 / 2} 2^{-\ell}\right)^{1 / 4}\left\|\left(\sum_{j, \nu, m}\left|\mathscr{F}_{\lambda, \ell}^{\nu, j, m}(f)\right|^{2}\right)^{1 / 2}\right\|_{L^{4}\left(\mathbb{R}^{3}\right)}^{2} \\
& +C_{\varepsilon N} \lambda^{-N}\|f\|_{L^{4}\left(\mathbb{R}^{2}\right)}^{2} .
\end{aligned}
$$

We now proceed as in $\S 4$ to control the left-hand side of $(6.11)$ by the twodimensional version of Carleson's square function $\left(\sum_{\kappa \in \mathbb{Z}^{2}}\left|P^{\kappa} f\right|^{2}\right)^{1 / 2}$. An integration by parts argument, just as in Lemma 4.7, shows that the kernel $K_{\lambda, \ell}^{\nu, j, m}$ satisfies the inequality kernel satisfies the pointwise bounds

$$
\left|K_{\lambda, \ell}^{\nu, j, m}(x, t, y)\right| \leqslant C_{N} \lambda\left(1+\lambda^{1 / 2}\left|\varphi_{\eta}^{\prime}\left(x, t, \eta_{\nu}\right)-y\right|\right)^{-N} .
$$

Observe also that if $K_{\lambda, \ell}^{\nu, j, m, \kappa}$ denotes the kernel of $\mathscr{F}_{\lambda, \ell}^{\nu, j, m} P^{\kappa}$, then there is $\kappa(x, t, \nu, j, m) \in \mathbb{Z}^{2}$ such that

$$
K_{\lambda, \ell}^{\nu, j, m, \kappa}(x, t, y)=0 \quad \text { if }|\kappa-\kappa(x, t, \nu, j, m)|>C_{0}
$$

for a uniform constant $C_{0}$. Applying (6.12) and (6.13) gives

$$
\begin{aligned}
& \sum_{j, \nu, m}\left|\mathscr{F}_{\lambda, \ell}^{\nu, j, m} f(x, t)\right|^{2} \\
& \quad \leqslant C \sum_{\nu, k} \int \lambda\left(1+\lambda^{1 / 2}\left|\varphi_{\eta}^{\prime}\left(x, t, \eta_{\nu}\right)-y\right|\right)^{-N}\left|P^{\kappa} f(y)\right|^{2} d y .
\end{aligned}
$$


Now we may put the previous estimates (6.9), (6.10) and (6.11) together and use duality to obtain

$$
\begin{aligned}
\left\|\mathscr{F}_{\lambda} f\right\|_{L^{4}\left(\mathbb{R}^{3}\right)} \leqslant & C_{\varepsilon} \lambda^{3 \varepsilon} \sup _{\|g\|_{2}=1}\left(\int_{\mathbb{R}^{3}} \sum_{\nu, j, m} \sum_{\kappa \in \mathbb{Z}^{2}}\left|\mathscr{F}_{\lambda, \ell}^{\nu, j, m} P^{\kappa} f(x, t)\right|^{2} g(x, t) d x d t\right)^{1 / 2} \\
& +\|f\|_{L^{4}\left(\mathbb{R}^{2}\right)^{.}}
\end{aligned}
$$

Let

$$
M_{\lambda^{-1 / 2}} g(y)=\sup _{\nu} \int_{\mathbb{R}^{3}} \lambda\left(1+\lambda^{1 / 2}\left|\varphi_{\eta}^{\prime}\left(x, t, \eta_{\nu}\right)-y\right|\right)^{-N}|g(x, t)| d x d t,
$$

and we see that the first term on the right hand side of $(6.14)$ is bounded by

$$
C_{N, \varepsilon} \lambda^{3 \varepsilon} \sup _{\|g\|_{2}=1}\left(\int_{\mathbb{R}^{2}} \sum_{\kappa \in \mathbb{Z}^{2}}\left|P^{\kappa} f(y)\right|^{2} M_{\lambda^{-1 / 2}} g(y) d y\right)^{1 / 2} \text {. }
$$

Now the proof of Theorem 6.1 is complete if we apply Theorem 5.1 and the square function estimate (4.24).

Orthogonality arguments. We are now going to prove Lemmas 6.6 and 6.7 . The proofs rely on the following integration by parts argument. One notices that the left-hand side of (6.6) equals the absolute value of

$$
\int \mathscr{H}_{j, k, j^{\prime}, k^{\prime}}^{\nu, \mu, \nu^{\prime}, \mu^{\prime}}\left(\eta, \xi, \eta^{\prime}, \xi^{\prime}\right) \hat{f}(\eta) \hat{f}(\xi) \overline{\hat{f}\left(\eta^{\prime}\right) \hat{f}\left(\xi^{\prime}\right)} d \eta d \xi d \eta^{\prime} d \xi^{\prime}
$$

where

$$
\begin{aligned}
& \mathscr{H}_{j, k, j^{\prime}, k^{\prime}}^{\nu, \mu, \nu^{\prime}, \mu^{\prime}}\left(\eta, \xi, \eta^{\prime}, \xi^{\prime}\right) \\
& \quad=\int e^{i \Phi\left(x, t, \eta, \xi, \eta^{\prime}, \xi^{\prime}\right)} b_{j, k, j^{\prime}, k^{\prime}}^{\nu, \mu, \mu^{\prime}}\left(x, t, \eta, \xi, \eta^{\prime}, \xi^{\prime}\right) d x d t
\end{aligned}
$$

with

$$
\Phi\left(x, t, \eta, \xi, \eta^{\prime}, \xi^{\prime}\right)=\varphi(x, t, \eta)+\varphi(x, t, \xi)-\varphi\left(x, t, \eta^{\prime}\right)-\varphi\left(x, t, \xi^{\prime}\right)
$$

and

$$
\begin{aligned}
& b_{j, k, j^{\prime}, k^{\prime}}^{\nu, \mu, \nu^{\prime}, \mu^{\prime}}\left(x, t, \eta, \xi, \eta^{\prime}, \xi^{\prime}\right) \\
& \quad=a_{\lambda, \ell}^{\nu, j}(x, t, \eta) a_{\lambda, \ell}^{\mu, k}(x, t, \xi) \overline{a_{\lambda, \ell}^{\nu^{\prime}, j^{\prime}}\left(x, t, \eta^{\prime}\right) a_{\lambda, \ell}^{\mu^{\prime}, k^{\prime}}\left(x, t, \xi^{\prime}\right)}
\end{aligned}
$$

We evaluate (6.16) using an integration by parts argument. If $\mathscr{L}$ denotes the adjoint of the operator $\left|\nabla_{x, t} \Phi\right|^{-2}\left\langle\nabla_{x, t} \Phi, \nabla_{x, t}\right\rangle$ then we have of course

$$
\begin{aligned}
& \mathscr{H}_{j, k, j^{\prime}, k^{\prime}}^{\nu, \mu, \nu^{\prime}, \mu^{\prime}}\left(\eta, \xi, \eta^{\prime} \xi^{\prime}\right) \\
& \quad=\int e^{i \Phi\left(x, t, \eta, \xi, \eta^{\prime}, \xi^{\prime}\right)} \mathscr{L}^{N} b_{j, k, j^{\prime}, k^{\prime}}^{\nu, \mu, \nu^{\prime}, \mu^{\prime}}\left(x, t, \eta, \xi, \eta^{\prime}, \xi^{\prime}\right) d x d t
\end{aligned}
$$

for those $\left(\eta, \xi, \eta^{\prime}, \xi^{\prime}\right)$ with $\nabla_{x, t} \Phi \neq 0$. 
A straightforward computation shows that $\mathscr{L}^{N} b_{j, k, j^{\prime}, k^{\prime}}^{\nu, \mu, \nu^{\prime}}\left(x, t, \eta, \xi, \eta^{\prime}, \xi^{\prime}\right)$ is the sum of expressions of the form

$$
\begin{gathered}
\frac{\prod_{i=0}^{N_{2}}\left(\psi_{i}(x, t, \eta)+\psi_{i}(x, t, \xi)-\psi_{i}\left(x, t, \xi^{\prime}\right)-\psi_{i}\left(x, t, \eta^{\prime}\right)\right)}{\left|\nabla_{x, t} \Phi\left(x, t, \eta, \xi, \eta^{\prime}, \xi^{\prime}\right)\right|^{N+N_{1}}} \\
\quad \times \partial_{x, t}^{\alpha} b_{j, k, j^{\prime}, k^{\prime}}^{\nu, \mu, \nu^{\prime}, \mu^{\prime}}\left(x, t, \eta, \xi, \eta^{\prime}, \xi^{\prime}\right)
\end{gathered}
$$

where $\psi_{i}$ are homogeneous functions of degree one and $0 \leqslant N_{1} \leqslant N+1$, $0 \leqslant N_{2} \leqslant N_{1},|\alpha| \leqslant N-N_{2}$. In order to evaluate (6.20) we first observe that

$$
\left|\partial_{x, t}^{\alpha} b_{j, k, j^{\prime}, k^{\prime}}^{\nu, \mu, \nu^{\prime}, \mu^{\prime}}\left(x, t, \eta, \xi, \eta^{\prime}, \xi^{\prime}\right)\right| \leqslant C_{\alpha} 2^{\ell|\alpha|} .
$$

Next we observe that there are positive constants $C_{0}, c$ independent of $\lambda$ such that if $\left|j+k-j^{\prime}-k^{\prime}\right| \geqslant C_{0}$ we have

$$
\begin{aligned}
& \left|\varphi_{t}^{\prime}(x, t, \eta)+\varphi_{t}^{\prime}(x, t, \xi)-\varphi_{t}^{\prime}\left(x, t, \eta^{\prime}\right)-\varphi_{t}^{\prime}\left(x, t, \xi^{\prime}\right)\right| \\
& \geqslant c \lambda^{1 / 2}\left|j+k-j^{\prime}-k^{\prime}\right| .
\end{aligned}
$$

Therefore by (6.20) and (6.21) it follows that $\mathscr{H}_{j, k, j^{\prime}, k^{\prime}}^{\nu, \mu, \nu^{\prime}, \mu^{\prime}}=O\left(\left|j+k-j^{\prime}-k^{\prime}\right|^{-N}\right)$ for every $N$, if $\left|j+k-j^{\prime}-k^{\prime}\right| \geqslant C_{0}$. Since the symbol $b_{j, k, j^{\prime}, k^{\prime}}^{\nu, \mu, \nu^{\prime}, \mu^{\prime}}$ is supported on a set of measure $2^{-4 \ell} \lambda^{6}$, we see that if $\left(j, k, j^{\prime}, k^{\prime}\right)$ is as in (6.6) then the integral (6.15) is bounded by $C_{\varepsilon, N} \lambda^{-N}\|f\|_{1}^{4}$. Since we may assume that $f$ is compactly supported, this completes the proof of (6.6). The proof of (6.7) follows from the same reasoning.

In order to prove Lemma 6.7 we observe that the left-hand side of (6.8) equals (6.15) with $j=j^{\prime}$ and $k=k^{\prime}$. Again we carry out the above integration by parts. In order to estimate the symbol $\mathscr{L}^{N} b_{j, k, j, k}^{\nu, \mu, \nu^{\prime}, \mu^{\prime}}$ we will have to use the following calculus lemma, which for later use (in the proof of Theorem 6.2) is formulated in more generality than needed here.

Lemma 6.8. Let $\psi \in C^{4}\left(\mathbb{R}^{2} \backslash 0\right)$ be homogeneous of degree one. Let $\delta<\pi / 4$, $a_{0}<1 / 4, A_{0} \geqslant 1$. Let $\mathscr{S}_{\lambda}$ be the intersection of a sector which subtends an angle of size $\delta$ with the annulus $\left\{\eta:\left(1-a_{0}\right) \lambda \leqslant|\eta| \leqslant\left(1+a_{0}\right) \lambda\right\}$. Let $h \in C^{1}\left(\mathbb{R}^{2} \backslash 0\right)$ be homogeneous of degree 1 such that $b_{0}|\eta| \leqslant h(\eta) \leqslant b_{1}|\eta|,\left|\nabla_{\eta} h(\eta)\right| \leqslant b_{2}$ for some positive constants $b_{0}, b_{1}, b_{2}$.

Suppose that $a_{0}^{-1} \leqslant 2^{n}, n \leqslant \ell, 2^{\ell} \leqslant \delta \lambda^{1 / 2}$, and that $\xi, \eta, \xi^{\prime}, \eta^{\prime} \in \mathscr{S}_{\lambda}$ are chosen such that for given integers $\nu, \mu, \nu^{\prime}, \mu^{\prime}$

(1) $\left|\arg (\eta)-\nu \lambda^{-1 / 2}\right| \leqslant \lambda^{-1 / 2} ;\left|\arg \left(\eta^{\prime}\right)-\nu^{\prime} \lambda^{-1 / 2}\right| \leqslant \lambda^{-1 / 2} ;$

(2) $\left|\arg (\xi)-\mu \lambda^{-1 / 2}\right| \leqslant \lambda^{-1 / 2},\left|\arg \left(\xi^{\prime}\right)-\mu^{\prime} \lambda^{-1 / 2}\right| \leqslant \lambda^{-1 / 2}$;

(3) $2^{\ell-1} \lambda^{-1 / 2} \leqslant \max \left\{|\arg (\eta)-\arg (\xi)|,\left|\arg \left(\eta^{\prime}\right)-\arg \left(\xi^{\prime}\right)\right|\right\} \leqslant 2^{\ell+1} \lambda^{-1 / 2}$;

(4) $\left|h(\eta)-h\left(\eta^{\prime}\right)\right| \leqslant 2^{-n} \lambda$;

(5) $\left|h(\xi)-h\left(\xi^{\prime}\right)\right| \leqslant 2^{-n} \lambda$.

Then one can choose $\delta, a_{0}$ sufficiently small, and $A_{0}$ sufficiently large (only depending on $\left.\psi, b_{0}, b_{1}, b_{2}\right)$ such that for all $\nu, \mu, \nu^{\prime}, \mu^{\prime}$ with $A_{0} 2^{\ell-n} \leqslant$ 
$\left|\nu-\nu^{\prime}\right|+\left|\mu-\mu^{\prime}\right| \leqslant \delta \lambda^{1 / 2}$

$$
\begin{aligned}
& \left|\psi(\eta)+\psi(\xi)-\psi\left(\eta^{\prime}\right)-\psi\left(\xi^{\prime}\right)\right| \\
& \quad \leqslant C\left[\left(2^{\ell}\left|\nu-\nu^{\prime}\right|+\left|\nu-\nu^{\prime}\right|^{2}\right)+\left(2^{\ell}\left|\mu-\mu^{\prime}\right|+\left|\mu-\mu^{\prime}\right|^{2}\right)+\left|\eta+\xi-\eta^{\prime}-\xi^{\prime}\right|\right] .
\end{aligned}
$$

Suppose now that $\psi$ satisfies the additional assumption rank $\psi_{\eta \eta}^{\prime \prime}=1$. Then if either $\mu \leqslant \nu$ and $\mu^{\prime} \leqslant \nu^{\prime}$ or $\nu \leqslant \mu$ and $\nu^{\prime} \leqslant \mu^{\prime}$ and if $A_{0} 2^{\ell-n} \leqslant\left|\nu-\nu^{\prime}\right|+$ $\left|\mu-\mu^{\prime}\right| \leqslant \delta \lambda^{1 / 2}$ we have also with suitable positive constants $c_{0}, C_{0}$

$$
\begin{aligned}
& \left|\psi(\eta)+\psi(\xi)-\psi\left(\eta^{\prime}\right)-\psi\left(\xi^{\prime}\right)\right| \\
& \quad \geqslant c_{0}\left[\left(2^{\ell}\left|\nu-\nu^{\prime}\right|+\left|\nu-\nu^{\prime}\right|^{2}\right)+\left(2^{\ell}\left|\mu-\mu^{\prime}\right|+\left|\mu-\mu^{\prime}\right|^{2}\right)\right]-C_{0}\left|\eta+\xi-\eta^{\prime}-\xi^{\prime}\right| .
\end{aligned}
$$

For later use (in the proof of Theorem 6.2) observe that the assumptions (1-5) and the right-hand sides of (6.22) and (6.23) are invariant under the change of variables $\left(\eta, \xi, \eta^{\prime}, \xi^{\prime}\right) \rightarrow\left(\xi^{\prime}, \eta^{\prime}, \xi, \eta\right)$. Therefore we also get for all $\nu, \mu, \nu^{\prime}, \mu^{\prime}$ with $A_{0} 2^{\ell-n} \leqslant\left|\nu-\mu^{\prime}\right|+\left|\mu-\nu^{\prime}\right| \leqslant \delta \lambda^{1 / 2}$

$$
\begin{aligned}
& \left|\psi(\eta)+\psi(\xi)-\psi\left(\eta^{\prime}\right)-\psi\left(\xi^{\prime}\right)\right| \\
& \quad \leqslant C\left[\left(2^{\ell}\left|\nu-\mu^{\prime}\right|+\left|\nu-\mu^{\prime}\right|^{2}\right)+\left(2^{\ell}\left|\mu-\nu^{\prime}\right|+\left|\mu-\nu^{\prime}\right|^{2}\right)+\left|\eta+\xi-\eta^{\prime}-\xi^{\prime}\right|\right] .
\end{aligned}
$$

Moreover, if rank $\psi_{\eta \eta}^{\prime \prime}=1$ and either $\mu \leqslant \nu$ and $\nu^{\prime} \leqslant \mu^{\prime}$ or $\nu \leqslant \mu$ and $\mu^{\prime} \leqslant \nu^{\prime}$ and if $A_{0} 2^{\ell-n} \leqslant\left|\nu-\mu^{\prime}\right|+\left|\mu-\nu^{\prime}\right| \leqslant \delta \lambda^{1 / 2}$ then

$$
\begin{aligned}
& \left|\psi(\eta)+\psi(\xi)-\psi\left(\eta^{\prime}\right)-\psi\left(\xi^{\prime}\right)\right| \\
& \quad \geqslant c_{0}\left[\left(2^{\ell}\left|\nu-\mu^{\prime}\right|+\left|\nu-\mu^{\prime}\right|^{2}\right)+\left(2^{\ell}\left|\mu-\nu^{\prime}\right|+\left|\mu-\nu^{\prime}\right|^{2}\right)\right]-C_{0}\left|\eta+\xi-\eta^{\prime}-\xi^{\prime}\right| .
\end{aligned}
$$

End of proof of Lemma 6.7. Since the decomposition of the symbol with respect to $j$ is done in terms of

$$
\varphi_{t}^{\prime}(x, t, \eta)=q\left(x, t, \varphi_{x}^{\prime}(x, t, \eta)\right)
$$

we want to change variables and apply Lemma 6.8 with $h=q$ for the new variables $\varphi_{x}^{\prime}(x, t, \eta)$. On the other hand, the angular decomposition was done in terms of $\eta$, independent of $(x, t)$. Since the diffeomorphism $\left.\eta \rightarrow \varphi_{x}^{\prime}(x, t, \eta)\right)$ is homogeneous, however, we have

$$
\left|\arg \left(\varphi_{x}^{\prime}(x, t, \eta)\right)-\arg \left(\varphi_{x}^{\prime}\left(x, t, \eta^{\prime}\right)\right)\right| \approx\left|\arg (\eta)-\arg \left(\eta^{\prime}\right)\right| .
$$

Therefore we may replace $\left|\eta+\xi-\eta^{\prime}-\xi^{\prime}\right|$ in $(6.22),(6.23)$ by $\left|\Phi_{x}^{\prime}\left(\eta, \xi, \eta^{\prime}, \xi^{\prime}\right)\right|$. Now if $j=j^{\prime}, k=k^{\prime}$, we can use $(6.21)$, (6.22) with $\psi=\psi_{i},(6.23)$ with $\psi=q$, and (6.26) to estimate the expression (6.20) by

$$
C 2^{\ell\left(N-N_{2}\right)} \frac{\left(\left|\Phi_{x}^{\prime}\right|+\left(2^{\ell}\left|\nu-\nu^{\prime}\right|+\left|\nu-\nu^{\prime}\right|^{2}\right)+\left(2^{\ell}\left|\mu-\mu^{\prime}\right|+\left|\mu-\mu^{\prime}\right|^{2}\right)\right)^{N_{2}}}{\left(\max \left\{\left|\Phi_{x}^{\prime}\right|,\left|\left(2^{\ell}\left|\nu-\nu^{\prime}\right|+\left|\nu-\nu^{\prime}\right|^{2}\right)+\left(2^{\ell}\left|\mu-\mu^{\prime}\right|+\left|\mu-\mu^{\prime}\right|^{2}\right)-\right| \Phi_{x}^{\prime}||\right\}\right)^{N+N_{1}}},
$$


which is $O\left(\left(\left|\nu-\nu^{\prime}\right|+\left|\mu-\mu^{\prime}\right|\right)^{-N}\right)$. Therefore, the integral (6.15) is $\leqslant$ $C_{\varepsilon, N} \lambda^{-N}\|f\|_{1}^{4}$ if $\left|\nu-\nu^{\prime}\right|+\left|\mu-\mu^{\prime}\right| \geqslant \lambda^{\varepsilon}$, and the proof of Lemma 6.7 is complete once we have proved Lemma 6.8.

Proof of Lemma 6.8. Using symmetry considerations we may assume that

$$
\left|\arg \left(\eta^{\prime}\right)-\arg \left(\xi^{\prime}\right)\right| \leqslant|\arg (\eta)-\arg (\xi)|,
$$

and, moreover, that $\mu \leqslant \nu$ and $\mu^{\prime} \leqslant \nu^{\prime}$. Using an appropriate rotation we may also assume that $\eta_{2}+\xi_{2}=0$ so that $\eta, \xi, \eta^{\prime}, \xi^{\prime}$ are supported in a narrow cone containing the $\eta_{1}$-axis. Observe that then $\eta_{2}>0$. Since $\tan x-\tan y \approx x-y$ for small $x, y$, we may replace $\arg (\eta), \arg (\xi), \arg \left(\eta^{\prime}\right), \arg \left(\xi^{\prime}\right)$ by $\eta_{2} / \eta_{1}$, $\xi_{2} / \xi_{1}, \eta_{2}^{\prime} / \eta_{1}^{\prime}$, and $\xi_{2}^{\prime} / \xi_{1}^{\prime}$, respectively, in the hypotheses (1), (2), and (3).

We write down the Taylor expansion of the function $s \rightarrow \psi(1, s)$ up to terms of third order and obtain using the homogeneity of the phase function

$$
\begin{aligned}
\psi(\eta)+ & \psi(\xi)-\psi\left(\eta^{\prime}\right)-\psi\left(\xi^{\prime}\right) \\
= & \eta_{1} \psi\left(1, \frac{\eta_{2}}{\eta_{1}}\right)+\xi_{1} \psi\left(1, \frac{\xi_{2}}{\xi_{1}}\right)-\eta_{1}^{\prime} \psi\left(1, \frac{\eta_{2}^{\prime}}{\eta_{1}^{\prime}}\right)-\xi_{1}^{\prime} \psi\left(1, \frac{\xi_{2}^{\prime}}{\xi_{1}^{\prime}}\right) \\
= & \left(\eta_{1}+\xi_{1}-\eta_{1}^{\prime}-\xi_{1}^{\prime}\right) \psi(1,0) \\
& +\left(\eta_{2}+\xi_{2}-\eta_{2}^{\prime}-\xi_{2}^{\prime}\right) \frac{\partial \psi}{\partial \eta_{2}}(1,0) \\
& +\left[\eta_{1}\left(\frac{\eta_{2}}{\eta_{1}}\right)^{2}+\xi_{1}\left(\frac{\xi_{2}}{\xi_{1}}\right)^{2}-\eta_{1}^{\prime}\left(\frac{\eta_{2}^{\prime}}{\eta_{1}^{\prime}}\right)^{2}-\xi_{1}^{\prime}\left(\frac{\xi_{2}^{\prime}}{\xi_{1}^{\prime}}\right)^{2}\right] \frac{1}{2} \frac{\partial^{2} \psi}{\partial \eta_{2}^{2}}(1,0) \\
& +\left[\eta_{1} r\left(\frac{\eta_{2}}{\eta_{1}}\right)\left(\frac{\eta_{2}}{\eta_{1}}\right)^{2}+\xi_{1} r\left(\frac{\xi_{2}}{\xi_{1}}\right)\left(\frac{\xi_{2}}{\xi_{1}}\right)^{2}-\eta_{1}^{\prime} r\left(\frac{\eta_{2}^{\prime}}{\eta_{1}^{\prime}}\right)\left(\frac{\eta_{2}^{\prime}}{\eta_{1}^{\prime}}\right)^{2}-\xi_{1}^{\prime} r\left(\frac{\xi_{2}^{\prime}}{\xi_{1}^{\prime}}\right)\left(\frac{\xi_{2}^{\prime}}{\xi_{1}^{\prime}}\right)^{2}\right]
\end{aligned}
$$

where

$$
r(t)=\frac{t}{2} \int_{0}^{1}(1-s)^{2} \frac{\partial^{3} \psi}{\partial \eta_{2}^{3}}(1, s t) d s .
$$

We now set $\alpha=\xi_{2} / \xi_{1}, \beta=\eta_{2} / \eta_{1}, \alpha^{\prime}=\xi_{2}^{\prime} / \xi_{1}^{\prime}, \beta^{\prime}=\eta_{2}^{\prime} / \eta_{1}^{\prime}$. It suffices to show the estimates

$$
\begin{aligned}
& \left|\eta_{1} \beta^{2}+\xi_{1} \alpha^{2}-\eta_{1}^{\prime} \beta^{\prime 2}-\xi_{1}^{\prime} \alpha^{\prime 2}\right| \\
& \quad \leqslant C\left[\left(2^{\ell}\left|\nu-\nu^{\prime}\right|+\left|\nu-\nu^{\prime}\right|^{2}\right)+\left(2^{\ell}\left|\mu-\mu^{\prime}\right|+\left|\mu-\mu^{\prime}\right|^{2}\right)+\left|\eta+\xi-\eta^{\prime}-\xi^{\prime}\right|\right],
\end{aligned}
$$

$$
\begin{aligned}
& \left|\eta_{1} r(\beta) \beta^{2}+\xi_{1} r(\alpha) \alpha^{2}-\eta_{1}^{\prime} r\left(\beta^{\prime}\right) \beta^{\prime 2}-\xi_{1}^{\prime} r\left(\alpha^{\prime}\right) \alpha^{\prime 2}\right| \\
& \quad \leqslant C\left(A_{0}^{-1}+\delta\right)\left[\left(2^{\ell}\left|\nu-\nu^{\prime}\right|+\left|\nu-\nu^{\prime}\right|^{2}\right)+\left(2^{\ell}\left|\mu-\mu^{\prime}\right|+\left|\mu-\mu^{\prime}\right|^{2}\right)\right] \\
& \quad+C^{\prime}\left|\eta+\xi-\eta^{\prime}-\xi^{\prime}\right|,
\end{aligned}
$$


and

$$
\begin{aligned}
\mid \eta_{1} \beta^{2} & +\xi_{1} \alpha^{2}-\eta_{1}^{\prime} \beta^{\prime 2}-\xi_{1}^{\prime} \alpha^{\prime 2} \mid \\
\geqslant & c_{0}\left[\left(2^{\ell}\left|\nu-\nu^{\prime}\right|+\left|\nu-\nu^{\prime}\right|^{2}\right)+\left(2^{\ell}\left|\mu-\mu^{\prime}\right|+\left|\mu-\mu^{\prime}\right|^{2}\right)\right] \\
& -C_{0}\left|\eta+\xi-\eta^{\prime}-\xi^{\prime}\right| .
\end{aligned}
$$

The terms on the left hand sides have a common form which suggests to use the following identity

$$
\begin{aligned}
c_{1} d_{1}+c_{2} d_{2}-c_{3} d_{3}-c_{4} d_{4}= & \frac{1}{4}\left(c_{1}+c_{2}+c_{3}+c_{4}\right)\left(d_{1}+d_{2}-d_{3}-d_{4}\right) \\
& +\frac{1}{4}\left(c_{1}-c_{2}+c_{3}-c_{4}\right)\left(d_{1}-d_{2}-d_{3}+d_{4}\right) \\
& +\frac{1}{4}\left(c_{1}+c_{2}-c_{3}-c_{4}\right)\left(d_{1}+d_{2}+d_{3}+d_{4}\right) \\
& +\frac{1}{4}\left(c_{1}-c_{2}-c_{3}+c_{4}\right)\left(d_{1}-d_{2}+d_{3}-d_{4}\right) .
\end{aligned}
$$

We first show (6.27) and write

$$
\eta_{1} \beta^{2}+\xi_{1} \alpha^{2}-\eta_{1}^{\prime} \beta^{\prime 2}-\xi_{1}^{\prime} \alpha^{\prime 2}=I+I I+I I I+I V
$$

where the four terms are the corresponding terms on the right-hand side of (6.30) with

$$
\left(c_{1}, c_{2}, c_{3}, c_{4}\right)=\left(\eta_{1}, \xi_{1}, \eta_{1}^{\prime}, \xi_{1}^{\prime}\right) \text { and }\left(d_{1}, d_{2}, d_{3}, d_{4}\right)=\left(\beta^{2}, \alpha^{2}, \beta^{\prime 2}, \alpha^{\prime 2}\right) \text {. }
$$

Since we are assuming that $\eta_{2}+\xi_{2}=0$, it is easy to see that $|\beta|,|\alpha| \approx 2^{\ell} \lambda^{-1 / 2}$ (provided $a_{0}$ is chosen small enough). Hence $\left|\beta+\beta^{\prime}\right| \leqslant C\left(2^{\ell}+\left|\nu-\nu^{\prime}\right|\right)$ and $\left|\alpha+\alpha^{\prime}\right| \leqslant C\left(2^{\ell}+\left|\mu-\mu^{\prime}\right|\right)$, and we see that

$\left|\beta^{2}-\beta^{\prime 2}\right| \leqslant C \frac{\left|\nu-\nu^{\prime}\right| 2^{\ell}+\left|\nu-\nu^{\prime}\right|}{\lambda^{1 / 2}}, \quad\left|\alpha^{2}-\alpha^{\prime 2}\right| \leqslant C \frac{\left|\mu-\mu^{\prime}\right|}{\lambda^{1 / 2}} \frac{2^{\ell}+\left|\mu-\mu^{\prime}\right|}{\lambda^{1 / 2}}$.

Therefore, we obtain

$$
\begin{gathered}
I \leqslant C\left[\left(2^{\ell}\left|\nu-\nu^{\prime}\right|+\left|\nu-\nu^{\prime}\right|^{2}\right)+\left(2^{\ell}\left|\mu-\mu^{\prime}\right|+\left|\mu-\mu^{\prime}\right|^{2}\right)\right] \\
I I \leqslant C a_{0}\left[\left(2^{\ell}\left|\nu-\nu^{\prime}\right|+\left|\nu-\nu^{\prime}\right|^{2}\right)+\left(2^{\ell}\left|\mu-\mu^{\prime}\right|+\left|\mu-\mu^{\prime}\right|^{2}\right)\right],
\end{gathered}
$$

as desired. On the other hand, the estimate for the third term is trivial:

$$
I I I \leqslant C\left|\eta_{1}+\xi_{1}-\eta_{1}^{\prime}-\xi_{1}^{\prime}\right| \leqslant C\left|\eta+\xi-\eta^{\prime}-\xi^{\prime}\right| .
$$

To handle $I V$ we first control $\left|c_{1}-c_{3}\right|$. If we write $\eta_{1}=h(\eta) / h(1, \beta)$ then our assumptions regarding $h$ leads to

$$
\begin{aligned}
\left|\eta_{1}-\eta_{1}^{\prime}\right| & =\left|\frac{h(\eta)}{h(1, \beta)}-\frac{h\left(\eta^{\prime}\right)}{h\left(1, \beta^{\prime}\right)}\right| \\
& =\left|\frac{h(\eta)-h\left(\eta^{\prime}\right)}{h(1, \beta)}+\frac{h\left(\eta^{\prime}\right)}{h(1, \beta) h\left(1, \beta^{\prime}\right)}\left(h\left(1, \beta^{\prime}\right)-h(1, \beta)\right)\right| \\
& \leqslant b_{0}^{-1}\left|h(\eta)-h\left(\eta^{\prime}\right)\right|+2 b_{1} b_{2} b_{0}^{-2} \lambda\left|\beta^{\prime}-\beta\right| .
\end{aligned}
$$


Since the same type of estimate holds for $\left|\xi_{1}-\xi_{1}^{\prime}\right|$, we get

$$
\left|\eta_{1}-\eta_{1}^{\prime}\right|+\left|\xi_{1}-\xi_{1}^{\prime}\right| \leqslant C\left(2^{-n} \lambda+\left(\left|\nu-\nu^{\prime}\right|+\left|\mu-\mu^{\prime}\right|\right) \lambda^{1 / 2}\right) .
$$

Observe also that, since $|\alpha|,|\beta| \leqslant C 2^{\ell} \lambda^{-1 / 2}$, we have

$$
\beta^{2}+\beta^{\prime 2} \leqslant C\left(2^{2 \ell} \lambda^{-1}+\left|\beta^{2}-{\beta^{\prime}}^{2}\right|\right), \quad \alpha^{2}+\alpha^{\prime 2} \leqslant C\left(2^{2 \ell} \lambda^{-1}+\left|\alpha^{2}-\alpha^{\prime 2}\right|\right) .
$$

Using (6.31) and (6.35) and our assumptions, $2^{\ell-n} \leqslant A_{0}^{-1}\left(\left|\nu-\nu^{\prime}\right|+\left|\mu-\mu^{\prime}\right|\right)$ and $2^{\ell} \leqslant \delta \lambda^{1 / 2}$, leads to

$$
\begin{aligned}
I V & \leqslant C 2^{\ell} \lambda^{-1}\left(2^{\ell}+\left|\nu-\nu^{\prime}\right|+\left|\mu-\mu^{\prime}\right|\right)\left(\lambda 2^{-n}+\left(\left|\nu-\nu^{\prime}\right|+\left|\mu-\mu^{\prime}\right|\right) \lambda^{1 / 2}\right) \\
& \leqslant C\left(A_{0}^{-1}+\delta\right)\left[\left(2^{\ell}\left|\nu-\nu^{\prime}\right|+\left|\nu-\nu^{\prime}\right|^{2}\right)+\left(2^{\ell}\left|\mu-\mu^{\prime}\right|+\left|\mu-\mu^{\prime}\right|^{2}\right)\right],
\end{aligned}
$$

which finishes the proof of (6.27).

In order to prove (6.28) we apply (6.30) with

$$
\begin{aligned}
\left(c_{1}, c_{2}, c_{3}, c_{4}\right) & =\left(\eta_{1} r(\eta), \xi_{1} r(\xi), \eta_{1}^{\prime} r\left(\eta^{\prime}\right), \xi_{1}^{\prime} r\left(\xi^{\prime}\right)\right), \\
\left(d_{1}, d_{2}, d_{3}, d_{4}\right) & =\left(\left(\eta_{2} / \eta_{1}\right)^{2},\left(\xi_{2} / \xi_{1}\right)^{2},\left(\eta_{2}^{\prime} / \eta_{1}^{\prime}\right)^{2},\left(\xi_{2}^{\prime}, \xi_{1}^{\prime}\right)^{2}\right)
\end{aligned}
$$

and obtain

$$
\begin{aligned}
& \left|\eta_{1} r(\beta) \beta^{2}+\xi_{1} r(\alpha) \alpha^{2}-\eta_{1}^{\prime} r\left(\beta^{\prime}\right) \beta^{\prime 2}-\xi_{1}^{\prime} r\left(\alpha^{\prime}\right) \alpha^{\prime 2}\right| \\
& \leqslant C \delta \lambda\left(\left|\beta^{2}-\beta^{\prime 2}\right|+\left|\alpha^{2}-\alpha^{\prime 2}\right|\right) \\
& +C\left(\left|\eta_{1} r(\beta)+\xi_{1} r(\alpha)-\eta_{1}^{\prime} r\left(\beta^{\prime}\right)-\xi_{1}^{\prime} r\left(\alpha^{\prime}\right)\right|\left(\beta^{2}+\beta^{\prime 2}+\alpha^{2}+\alpha^{\prime 2}\right)\right. \\
& +C\left(\beta^{2}+\beta^{\prime 2}-\alpha^{2}-\alpha^{\prime 2}\right) \\
& \times\left(\left|\eta_{1}-\eta_{1}^{\prime}\right|+\left|\xi_{1}-\xi_{1}^{\prime}\right|+\left|r(\beta)-r\left(\beta^{\prime}\right)\right|+\left|r(\alpha)-r\left(\alpha^{\prime}\right)\right|\right) .
\end{aligned}
$$

Now the first term in the right hand side (6.37) is estimated using (6.31). Since

$$
\left|r(\beta)-r\left(\beta^{\prime}\right)\right| \leqslant C\left|\beta-\beta^{\prime}\right|, \quad\left|r(\alpha)-r\left(\alpha^{\prime}\right)\right| \leqslant C\left|\alpha-\alpha^{\prime}\right|,
$$

the estimate for the third term is the same as for the term $I V$ in (6.36). For the second term one evaluates (6.30) with $\left(c_{1}, c_{2}, c_{3}, c_{4}\right)=\left(\eta_{1}, \xi_{1}, \eta_{1}^{\prime}, \xi_{1}^{\prime}\right)$ and $\left(d_{1}, d_{2}, d_{3}, d_{4}\right)=\left(r(\beta), r(\alpha), r\left(\beta^{\prime}\right), r\left(\alpha^{\prime}\right)\right)$ and uses similar arguments to obtain

$$
\begin{aligned}
& \left|\eta_{1} r(\beta)+\xi_{1} r(\alpha)-\eta_{1}^{\prime} r\left(\beta^{\prime}\right)-\xi_{1}^{\prime} r\left(\alpha^{\prime}\right)\right| \\
& \quad \leqslant C\left[\left(\left|\nu-\nu^{\prime}\right|+\left|\mu-\mu^{\prime}\right|\right) \lambda^{1 / 2}+2^{\ell-n} \lambda^{1 / 2}+\left|\eta_{1}+\xi_{1}-\eta_{1}^{\prime}-\xi_{1}^{\prime}\right|\right] .
\end{aligned}
$$

Now if one uses (6.31) again and puts the previous estimates together one obtains (6.28).

Finally we have to verify (6.29). In view of the estimates from above, (6.33)$(6.35)$, it is sufficient to show that

$$
\begin{aligned}
\left|\beta^{2}+\alpha^{2}-\beta^{\prime 2}-\alpha^{\prime 2}\right| \geqslant & c_{0} \lambda^{-1}\left[\left(2^{\ell}\left|\nu-\nu^{\prime}\right|+\left|\nu-\nu^{\prime}\right|^{2}\right)+\left(2^{\ell}\left|\mu-\mu^{\prime}\right|+\left|\mu-\mu^{\prime}\right|^{2}\right)\right] \\
& -C_{0} \lambda^{-1}\left|\eta+\xi-\eta^{\prime}-\xi^{\prime}\right| .
\end{aligned}
$$


The latter inequality is an immediate consequence of

$$
\begin{aligned}
\left|\left(\beta^{2}-\beta^{\prime 2}\right)-\left(\alpha^{2}-\alpha^{\prime 2}\right)\right| \leqslant C \lambda^{-1}[ & \left(\delta+a_{0}+A_{0}^{-1}\right)\left(2^{\ell}\left|\nu-\nu^{\prime}\right|+\left|\nu-\nu^{\prime}\right|^{2}\right) \\
& \left.+\left(2^{\ell}\left|\mu-\mu^{\prime}\right|+\left|\mu-\mu^{\prime}\right|^{2}\right)+\left|\eta+\xi-\eta^{\prime}-\xi^{\prime}\right|\right]
\end{aligned}
$$

and

$$
\begin{gathered}
\left|\beta^{2}-\beta^{\prime 2}\right|+\left|\alpha^{2}-\alpha^{\prime 2}\right| \geqslant c_{0} \lambda^{-1}\left(\left(2^{\ell}\left|\nu-\nu^{\prime}\right|+\left|\nu-\nu^{\prime}\right|^{2}\right)+\left(2^{\ell}\left|\mu-\mu^{\prime}\right|+\left|\mu-\mu^{\prime}\right|^{2}\right)\right) \\
-C_{0} \lambda^{-1}\left|\eta+\xi-\eta^{\prime}-\xi^{\prime}\right| .
\end{gathered}
$$

First we show (6.41). We write the left hand side as

$$
\begin{aligned}
& \frac{1}{2}\left(\xi_{2}^{\prime 2}-\xi_{2}^{2}-\eta_{2}^{2}+\eta_{2}^{\prime 2}\right)\left(\frac{1}{\xi_{1}^{\prime 2}}-\frac{1}{{\eta_{1}^{\prime}}^{2}}\right)+\frac{1}{2}\left({\xi_{2}^{\prime 2}}^{2}-\xi_{2}{ }^{2}+\eta_{2}{ }^{2}-\eta_{2}^{\prime 2}\right)\left(\frac{1}{\xi_{1}^{\prime 2}}+\frac{1}{{\eta_{1}^{\prime}}^{2}}\right) \\
& +\frac{\eta_{1}^{\prime 2}-\eta_{1}^{2}}{\eta_{1}^{\prime 2}}\left(\frac{\eta_{2}}{\eta_{1}}\right)^{2}+\frac{\xi_{1}^{2}-\xi_{1}^{\prime 2}}{\xi_{1}^{\prime 2}}\left(\frac{\xi_{2}}{\xi_{1}}\right)^{2}=I^{\prime}+I I^{\prime}+I I I^{\prime}+I V^{\prime} \text {. }
\end{aligned}
$$

Since $|\beta|,|\alpha| \approx 2^{\ell} \lambda^{-1 / 2}$, we get by (6.35)

$$
\begin{aligned}
\left|I I I^{\prime}+I V^{\prime}\right| & \leqslant C 2^{2 \ell} \lambda^{-2}\left(\left|\eta_{1}-\eta_{1}^{\prime}\right|+\left|\xi_{1}-\xi_{1}^{\prime}\right|\right) \\
& \leqslant C \lambda^{-1}\left(2^{2 \ell-n}+2^{\ell} \delta\left|\nu-\nu^{\prime}\right|+2^{\ell} \delta\left|\mu-\mu^{\prime}\right|\right)
\end{aligned}
$$

which is bounded by the right-hand side of (6.41).

Since $\eta_{2}+\xi_{2}=0$, we have

$$
\left|I I^{\prime}\right|=\left|\left(\xi_{2}^{\prime}+\eta_{2}^{\prime} r\right)\left(\xi_{2}^{\prime}-\eta_{2}^{\prime}\right)\left(\frac{1}{\xi_{1}^{\prime 2}}+\frac{1}{{\eta_{1}^{\prime}}^{2}}\right)\right| \leqslant C \lambda^{-1}\left|\eta+\xi-\eta^{\prime}-\xi^{\prime}\right| .
$$

Finally we rewrite $I^{\prime}$ as

$$
\begin{aligned}
I^{\prime}=\frac{1}{2}\left(\frac{1}{\xi_{1}^{\prime 2}}-\frac{1}{\eta_{1}^{\prime 2}}\right)[ & \xi_{1}^{\prime 2}\left(\frac{\xi_{2}^{\prime 2}}{\xi_{1}^{\prime 2}}-\frac{\xi_{2}^{2}}{\xi_{1}^{2}}\right)+\frac{\xi_{2}^{2}}{\xi_{1}^{2}}\left(\xi_{1}^{\prime 2}-\xi_{1}^{2}\right) \\
& \left.+\eta_{1}^{\prime 2}\left(\frac{\eta_{2}^{\prime 2}}{{\eta_{1}^{\prime}}^{2}}-\frac{\eta_{2}^{2}}{\eta_{1}^{2}}\right)+\frac{\eta_{2}^{2}}{\eta_{1}^{2}}\left(\eta_{1}^{\prime 2}-\eta_{1}^{2}\right)\right] .
\end{aligned}
$$

Since by assumption

$$
\left|\frac{1}{\xi_{1}^{\prime 2}}-\frac{1}{\eta_{1}^{\prime 2}}\right| \leqslant \frac{C}{\lambda^{3}}\left|\eta_{1}^{\prime}-\xi_{1}^{\prime}\right| \leqslant \frac{C^{\prime}}{\lambda^{2}}\left(\delta+a_{0}\right)
$$

we can apply the previous estimates again and obtain (6.41).

Next, in order to prove $(6.42)$ we first note that

$$
\begin{aligned}
& \left|\beta+\beta^{\prime}\right| \approx 2^{\ell}+\left|\nu-\nu^{\prime}\right| \quad \text { if } \operatorname{sign} \beta=\operatorname{sign} \beta^{\prime}, \\
& \left|\alpha+\alpha^{\prime}\right| \approx 2^{\ell}+\left|\mu-\mu^{\prime}\right| \quad \text { if } \operatorname{sign} \alpha=\operatorname{sign} \alpha^{\prime} .
\end{aligned}
$$


Therefore, in the case that both $\operatorname{sign} \beta=\operatorname{sign} \beta^{\prime}$ and $\operatorname{sign} \alpha=\operatorname{sign} \alpha^{\prime},(6.42)$ immediately follows. Secondly, the case $\operatorname{sign} \beta \neq \operatorname{sign} \beta^{\prime}$ and $\operatorname{sign} \alpha \neq \operatorname{sign} \alpha^{\prime}$ does not occur since we assume that $\operatorname{sign} \beta \neq \operatorname{sign} \alpha$ and $\mu \leqslant \nu, \mu^{\prime} \leqslant \nu^{\prime}$.

Finally, by a symmetry consideration it is enough to consider the case $\operatorname{sign} \beta$ $\neq \operatorname{sign} \beta^{\prime}$ and $\operatorname{sign} \alpha=\operatorname{sign} \alpha^{\prime}$. We distinguish three possibilities, namely,

(1) $\left|\nu-\nu^{\prime}\right| \geqslant 10 \cdot 2^{\ell}$,

(2) $\left|\nu-\nu^{\prime}\right| \leqslant 10 \cdot 2^{\ell}$ and $\left|\mu-\mu^{\prime}\right| \geqslant\left|\nu-\nu^{\prime}\right| / 10$,

(3) $\left|\nu-\nu^{\prime}\right| \leqslant 10 \cdot 2^{\ell}$ and $\left|\mu-\mu^{\prime}\right| \leqslant\left|\nu-\nu^{\prime}\right| / 10$.

In the first case we have, like in (6.43), $\left|\beta+\beta^{\prime}\right| \approx 2^{\ell}+\left|\nu-\nu^{\prime}\right|$, and the lower bound for $\left|\beta^{2}-\beta^{\prime 2}\right|$ follows as before while the lower bound for $\left|\alpha^{2}-\alpha^{\prime 2}\right|$ follows by $(6.44)$. The latter is already sufficient in the second case. In the last case we obtain no favorable positive lower bound, but now we can show that

$$
\left|\eta+\xi-\eta^{\prime}-\xi^{\prime}\right| \geqslant c_{0} 2^{\ell} \lambda^{1 / 2}
$$

which implies (6.42) in this case. In order to see (6.45) we apply (6.30) with $\left(c_{1}, c_{2}, c_{3}, c_{4}\right)=\left(\eta_{1}, \xi_{1}, \eta_{1}^{\prime}, \xi_{1}^{\prime}\right)$ and $\left(d_{1}, d_{2}, d_{3}, d_{4}\right)=\left(\beta, \alpha, \beta^{\prime}, \alpha^{\prime}\right)$ and see that

$$
\eta_{2}+\xi_{2}-\eta_{2}^{\prime}-\xi_{2}^{\prime}=\left(\eta_{1}+\xi_{1}+\eta_{1}^{\prime}+\xi_{1}^{\prime}\right)\left(\beta-\beta^{\prime}+\alpha-\alpha^{\prime}\right)+\rho\left(\beta, \alpha, \beta^{\prime}, \alpha^{\prime}\right)
$$

where in the present case the remainder $\rho\left(\beta, \alpha, \beta^{\prime}, \alpha^{\prime}\right)$ is $O\left(a_{0} 2^{\ell} \lambda^{1 / 2}\right)$. Since now also $\left|\beta-\beta^{\prime}+\alpha-\alpha^{\prime}\right| \geqslant 2^{\ell-2} \lambda^{1 / 2}$, we obtain (6.45) and then also the desired estimate (6.40), provided we choose $\delta, a_{0}$ small enough and $A_{0}$ large enough. This finishes the proof.

Proof of Theorem 6.2. Let $\mathscr{F}_{\lambda}$ be as in (4.5) and subject to the same assumptions on the symbol $a_{\lambda}$ as in the proof of Theorem 6.1. Then we shall prove the inequality

$$
\left\|\mathscr{F}_{\lambda} f\right\|_{L^{4}\left(L^{2}\right)} \leqslant C_{\varepsilon} \lambda^{\varepsilon}\|f\|_{L^{4}\left(\mathbb{R}^{2}\right)}
$$

for any $\varepsilon>0$. This implies the statement of Theorem 6.2 in the case $p=q=4$. The general case follows by interpolation, using the result of Theorem 3.2 for the case $p=2, q=6$.

In order to prove the $L^{4}$ estimate we may also assume that $a_{\lambda}$ is supported in a cone which subtends an angle less than $\lambda^{-\varepsilon}$. This only leads to an additional blowup of the constants by a factor of $\lambda^{\varepsilon}$. We now make an angular decomposition $\mathscr{F}_{\lambda}=\sum_{\nu} \mathscr{F}_{\lambda}^{\nu}$ as in the proof of Theorem 6.1 and observe that for indices $\nu, \mu$ in this sum we have $|\mu-\nu| \leqslant \lambda^{1 / 2-\varepsilon}$.

As in the proof of Theorem 6.1 we need to decompose the symbol $a_{\lambda}^{\nu}=$ $\chi_{\nu} a_{\lambda}=\sum_{j} a_{\lambda, r}^{\nu, j}$ where $a_{\lambda, r}^{\nu, j}$ is as in (6.4). Then $a_{\lambda, r}^{\nu, j}$ is supported in a shell where $\left|\varphi_{t}^{\prime}-j\right| \leqslant C 2^{-r} \lambda$. In the present proof we will use this decomposition only if $2^{r}>\lambda^{1 / 4}$.

If $2^{r} \leqslant \lambda^{1 / 4}$, we replace the decomposition in terms of $\varphi_{t}^{\prime}$ by a radial one 
which does not depend on $(x, t)$. We then set, for $0<\varepsilon_{0} \ll \varepsilon$,

$$
\begin{array}{ll}
\tilde{a}_{\lambda, r}^{\nu, j}(x, t, \eta)=\chi_{\nu}(\eta) \rho\left(\lambda^{-1 / 2} 2^{-r}|\eta|-j\right) a_{\lambda}(x, t, \eta) & \text { if } 1 \leqslant 2^{r} \leqslant \lambda^{\varepsilon_{0}}, \\
\tilde{a}_{\lambda, r}^{\nu, j}(x, t, \eta)=\chi_{\nu}(\eta) \rho\left(\lambda^{-1 / 2+\varepsilon_{0}} 2^{-r}|\eta|-j\right) a_{\lambda}(x, t, \eta) & \text { if } \lambda^{\varepsilon_{0}} \leqslant 2^{r} \leqslant \lambda^{1 / 4}
\end{array}
$$

and define the operators

$$
\begin{aligned}
& \mathscr{F}_{\lambda, r}^{\nu, j} f(x, t)=\int e^{i \varphi(x, t, \eta)} a_{\lambda, r}^{\nu, j}(x, t, \eta) \hat{f}(\eta) d \eta, \\
& \widetilde{F}_{\lambda, r}^{\nu, j} f(x, t)=\int e^{i \varphi(x, t, \eta)} \tilde{a}_{\lambda, r}^{\nu, j}(x, t, \eta) \hat{f}(\eta) d \eta .
\end{aligned}
$$

The main inequality we want to prove is

$$
\begin{aligned}
\|\mathscr{F} \lambda\|_{L^{4}\left(\mathbb{R}^{2}, L^{2}(\mathbb{R})\right)} \leqslant & C_{\varepsilon} \lambda^{\varepsilon} \sum_{1 \leqslant 2^{r} \leqslant \lambda^{1 / 4}}\left\|\left(\sum_{\nu, j}\left|\widetilde{F}_{\lambda, r}^{\nu, j} f\right|^{2}\right)^{1 / 2}\right\|_{L^{4}\left(\mathbb{R}^{3}\right)} \\
& +C_{\varepsilon} \lambda^{\varepsilon} \sum_{\lambda^{1 / 4} \leqslant 2^{r} \leqslant \lambda^{1 / 2-\varepsilon}}\left\|\left(\sum_{\nu, j}\left|\mathscr{F}_{\lambda, r}^{\nu, j} f\right|^{2}\right)^{1 / 2}\right\|_{L^{4}\left(\mathbb{R}^{3}\right)} \\
& +C_{\varepsilon, N} \lambda^{-N}\|f\|_{L^{1}\left(\mathbb{R}^{2}\right)}
\end{aligned}
$$

We shall first show how (6.47) can be used to complete the proof of the theorem. First, since we can assume that $f$ is compactly supported the $L^{1}$ norm is, of course, controlled by the $L^{4}$ norm. Next we want to show that

$$
\begin{aligned}
& \left\|\left(\sum_{\nu, j}\left|\mathscr{F}_{\lambda, r}^{\nu, j} f\right|^{2}\right)^{1 / 2}\right\|_{L^{4}\left(\mathbb{R}^{3}\right)} \leqslant C_{\varepsilon} \lambda^{\varepsilon}\|f\|_{L^{4}\left(\mathbb{R}^{3}\right)}, \\
& \left\|\left(\sum_{\nu, j}\left|\widetilde{\mathscr{F}}_{\lambda, r}^{\nu, j} f\right|^{2}\right)^{1 / 2}\right\|_{L^{4}\left(\mathbb{R}^{3}\right)} \leqslant C_{\varepsilon} \lambda^{\varepsilon}\|f\|_{L^{4}\left(\mathbb{R}^{3}\right)} .
\end{aligned}
$$

We only show (6.48) since the proof of (6.49) is similar. We make a decomposition of each sector $S^{\nu}=\operatorname{supp}_{\eta} \chi_{\nu}$ which does not depend on $(x, t)$. Set, with $\rho$ as in (6.4),

$$
\beta_{\lambda, r}^{\kappa}(\eta)=\rho\left(\lambda^{-1} 2^{r}\left\langle\eta, \eta_{\nu}\right\rangle-\kappa\right)
$$

and define $Q_{\lambda, r}^{\nu, \kappa}$ by

$$
\widehat{Q_{\lambda, r}^{\nu, \kappa} f}=\chi_{S^{\nu}}(\eta) \beta_{\lambda, r}^{\kappa}(\eta) \hat{f}(\eta) .
$$

We shall use a square function estimate that is due to Córdoba [13], namely,

$$
\left\|\left(\sum_{\nu, \kappa}\left|Q_{\lambda, r}^{\nu, \kappa} f\right|^{2}\right)^{1 / 2}\right\|_{L^{4}\left(\mathbb{R}^{2}\right)} \leqslant C(\log \lambda)^{c}\|f\|_{L^{4}\left(\mathbb{R}^{2}\right)}
$$

for some positive constant $c$. On account of (6.50) we would be done if we could show that

$$
\left\|\left(\sum_{\nu, j}\left|\mathscr{F}_{\lambda, r}^{\nu, j} f\right|^{2}\right)^{1 / 2}\right\|_{L^{4}\left(\mathbb{R}^{3}\right)} \leqslant C \lambda^{\varepsilon}\left\|\left(\sum_{\nu, \kappa}\left|Q_{\lambda, r}^{\nu, \kappa} f\right|^{2}\right)^{1 / 2}\right\|_{L^{4}\left(\mathbb{R}^{2}\right)} .
$$


To this end set

$$
\mathscr{F}_{\lambda, r}^{\nu, j, \kappa} f(x, t)=\int e^{i \varphi(x, t, \eta)} a_{\lambda, r}^{\nu, j}(x, t, \eta) \beta_{\lambda, r}^{\kappa}(\eta) \hat{f}(\eta) d \eta .
$$

Then

$$
\mathscr{F}_{\lambda, r}^{\nu, j}(f)=\sum_{\kappa} \mathscr{F}_{\lambda, r}^{\nu, j, \kappa} Q_{\lambda, r}^{\nu, \kappa} f
$$

Observe also that if $K_{\lambda, r}^{\nu, j, \kappa}$ denotes the kernel of $\mathscr{F}_{\lambda, r}^{\nu, j, \kappa}$, then there are integers $\kappa(x, t, \nu, j)$ such that

$$
K_{\lambda, r}^{\nu, j}(x, t, y)=0 \quad \text { if }|\kappa-\kappa(x, t, \nu, j)|>C_{0}
$$

for a uniform constant $C_{0}$. Next an integration by parts argument as in [28] shows that the kernels satisfy the pointwise bounds

$$
\begin{aligned}
\left|K_{\lambda, r}^{\nu, j, \kappa}(x, t, y)\right| \leqslant & C_{N} \frac{2^{-r} \lambda}{\left(1+2^{-r} \lambda\left|\left\langle\varphi_{\eta}^{\prime}\left(x, t, \eta_{\nu}\right)-y, \eta_{\nu}\right\rangle\right|\right)^{N}} \\
& \times \frac{\lambda^{1 / 2}}{\left(1+\lambda^{1 / 2}\left|\left\langle\varphi_{\eta}^{\prime}\left(x, t, \eta_{\nu}\right)-y, \eta_{\nu}^{\perp}\right\rangle\right|\right)^{N}}
\end{aligned}
$$

where the unit vector $\eta_{\nu}^{\perp}$ is orthogonal to $\eta_{\nu}$. In particular, we see that

$$
\int\left|K_{\lambda, \ell}^{\nu, j, \kappa}(x, t, y)\right| d y \leqslant C .
$$

Using (6.52) and (6.54) the square of the right-hand side of $(6.51)$ is bounded by

$$
\begin{aligned}
\sup _{\|g\|_{2}=1} \mid & \sum_{\nu, j, \kappa} \int_{\mathbb{R}^{3}}\left|\mathscr{F}_{\lambda, r}^{\nu, j, \kappa} Q_{\lambda, r}^{\nu, \kappa} f(x, t)\right|^{2} g(x, t) d x d t \mid \\
\leqslant C \lambda^{2 \varepsilon} & \sup _{\|g\|_{2}=1} \int_{\mathbb{R}^{2}} \sum_{\nu, \kappa}\left|Q_{\lambda, r}^{\nu, \kappa} f\right|^{2} \\
& \times \sup _{\nu}\left\{\int_{\mathbb{R}^{3}} \sup _{j, \kappa}\left|K_{\lambda, r}^{\nu, j}(x, t, y)\right||g(x, t)| d x d t\right\} d y .
\end{aligned}
$$

Now the proof of $(6.51)$ is complete once we have established the maximal inequality

$$
\begin{aligned}
& \left(\int_{\mathbb{R}^{2}} \sup _{\nu}\left|\int_{\mathbb{R}^{3}} \sup _{j, \kappa}\right| K_{\lambda, r}^{\nu, j}(x, t, y)|g(x, t) d x d t|^{2} d y\right)^{1 / 2} \\
& \quad \leqslant C_{\varepsilon} \lambda^{\varepsilon}\|g\|_{L^{2}\left(\mathbb{R}^{3}\right)}, \quad \varepsilon>0,
\end{aligned}
$$

But this is a consequence of (6.53) and Theorem 5.3. 
Proof of (6.47). Using the Cauchy-Schwarz inequality we get immediately $(6.55)$

$$
\begin{aligned}
& \left\|\mathscr{F}_{\lambda} f\right\|_{L^{4}\left(L^{2}\right)} \leqslant C_{\varepsilon} \lambda^{\varepsilon}\left\|\left(\sum_{\nu}\left|\mathscr{F}_{\lambda}^{\nu} f\right|^{2}\right)^{1 / 2}\right\|_{L^{4}\left(L^{2}\right)} \\
& +\sum_{\lambda^{\varepsilon} \leqslant 2^{r} \leqslant \lambda^{1 / 2-\varepsilon}}\left(\int\left|\int \sum_{\substack{\left|\nu-\nu^{\prime}\right| \approx 2^{r} \lambda^{\varepsilon} \\
\nu^{\prime} \leqslant \nu}} \mathscr{F}_{\lambda}^{\nu} f \overline{\mathscr{F}_{\lambda}^{\nu^{\prime}} f} d t\right|^{2} d x\right)^{1 / 4} \\
& \leqslant C_{\varepsilon} \lambda^{\varepsilon} \sum_{2^{r} \leqslant \lambda^{\varepsilon}}\left\|\left(\sum_{\nu, j}\left|\mathscr{F}_{\lambda, r}^{\nu, j} f\right|^{2}\right)^{1 / 2}\right\|_{L^{4}\left(\mathbb{R}^{3}\right)} \\
& +C_{\varepsilon}\left[\sum _ { \lambda ^ { \varepsilon } \leqslant 2 ^ { r } \leqslant \lambda ^ { 1 / 4 } } \left(\int \mid \int \sum_{\substack{\left|j-j^{\prime}\right| \leqslant C_{1}\left|\nu-\nu^{\prime}\right| \approx 2^{r} \\
\nu^{\prime} \leqslant \nu}} \widetilde{\mathscr{F}}_{\lambda, r}^{\nu, j} f(x, t)\right.\right.
\end{aligned}
$$

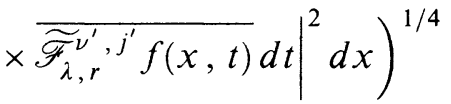

$$
\begin{aligned}
& +\sum_{\lambda^{1 / 4} \leqslant 2^{r} \leqslant \lambda^{1 / 2-\varepsilon}}\left(\int \mid \int \sum_{\left|j-j^{\prime}\right| \leqslant C_{1} \mid \begin{array}{c}
\left|\nu-\nu^{\prime}\right| \approx 2^{r} \\
\nu^{\prime} \leqslant \nu
\end{array}} \mathscr{F}_{\lambda, r}^{\nu, j} f(x, t)\right.
\end{aligned}
$$

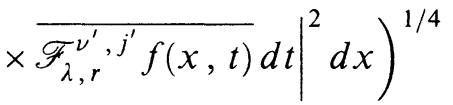

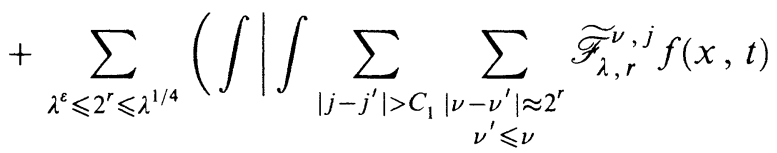

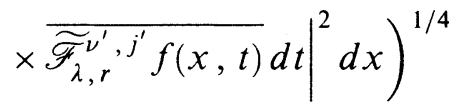

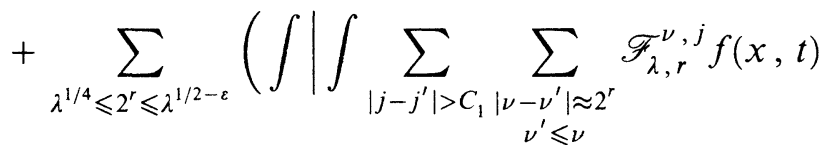

$$
\begin{aligned}
& \left.\left.\times\left.\overline{\mathscr{F}_{\lambda, r}^{\nu^{\prime}, j^{\prime}} f(x, t)} d t\right|^{2} d x\right)^{1 / 4}\right] .
\end{aligned}
$$

The reduction of (6.55) to (6.47) will depend on several orthogonality arguments. The first one shows that the last two terms in (6.55) are negligible provided $C_{1}$ is chosen sufficiently large (but independent of $\lambda$ ). The argument only depends on the assumption that $\varphi_{t}^{\prime}(x, t, \eta) \neq 0$ for $\eta \neq 0$.

Lemma 6.9. There is a constant $C_{1}$, independent of $\lambda$ such that if $\left|j-j^{\prime}\right| \geqslant C_{1}$

$$
\left|\int \mathscr{F}_{\lambda, r}^{\nu, j} f(x, t) \overline{\mathscr{F}_{\lambda, r}^{\nu^{\prime}, j^{\prime}} f(x, t)} d t\right| \leqslant C_{N} \lambda^{3} 2^{-2 r}\left(\lambda^{1 / 2} 2^{-r}\left|j-j^{\prime}\right|\right)^{-N}\|f\|_{1}^{2} .
$$

Suppose that $2^{r} \leqslant \lambda^{1 / 4},\left|\nu-\nu^{\prime}\right| \approx 2^{r}$, and $\left|j-j^{\prime}\right| \geqslant C_{1}$. Then if $C_{1}$ is 
sufficiently large,

$$
\begin{aligned}
& \left|\int \widetilde{\mathscr{F}}_{\lambda, r}^{\nu, j} f(x, t) \overline{\widetilde{\mathscr{F}}_{\lambda, r}^{\nu^{\prime}, j^{\prime}} f(x, t)} d t\right| \\
& \quad \leqslant C_{N} \lambda^{2} 2^{2 r}\left(2^{r}\left|j-j^{\prime}\right|\right)^{-N}\|f\|_{1}^{2} \quad \text { if } 1 \leqslant 2^{r} \leqslant \lambda^{\varepsilon_{0}}, \\
& \left|\int \widetilde{\mathscr{F}}_{\lambda, r}^{\nu, j} f(x, t) \overline{\widetilde{\mathscr{F}}_{\lambda, r}^{\nu^{\prime}, j^{\prime}} f(x, t)} d t\right| \\
& \quad \leqslant C_{N} \lambda^{2} 2^{2 r}\left(2^{r} \lambda^{-\varepsilon_{0}}\left|j-j^{\prime}\right|\right)^{-N}\|f\|_{1}^{2} \quad \text { if } \lambda^{\varepsilon_{0}} \leqslant 2^{r} \leqslant \lambda^{1 / 4} .
\end{aligned}
$$

Proof. Note that if $a_{\lambda, r}^{\nu, j}(x, t, \eta) \neq 0, a_{\lambda, r}^{\nu^{\prime}, j^{\prime}}\left(x, t, \eta^{\prime}\right) \neq 0$, and $\left|j-j^{\prime}\right|>4$, then

$$
\left|\varphi_{t}^{\prime}(x, t, \eta)-\varphi_{t}^{\prime}\left(x, t, \eta^{\prime}\right)\right| \geqslant c_{0} \lambda 2^{-r}
$$

without any restrictions on $\nu, \nu^{\prime}$. Now suppose that $\left|\nu-\nu^{\prime}\right| \leqslant 2^{r}$, $\tilde{a}_{\lambda, r}^{\nu, j}(x, t, \eta) \neq 0, \tilde{a}_{\lambda, r}^{\nu^{\prime}, j^{\prime}}\left(x, t, \eta^{\prime}\right) \neq 0$, and $\left|j-j^{\prime}\right|>C_{1}$. Since for $|\eta|,\left|\eta^{\prime}\right| \approx$ $\lambda$

$$
\left|\varphi_{t}^{\prime}(x, t, \eta)-\varphi_{t}^{\prime}\left(x, t, \eta^{\prime}\right)\right| \geqslant c_{0}\left\|\eta|-| \eta^{\prime}\right\|-C_{0} \lambda\left|\frac{\eta}{|\eta|}-\frac{\eta^{\prime}}{\left|\eta^{\prime}\right|}\right|,
$$

we get

$$
\begin{array}{ll}
\left|\varphi_{t}^{\prime}(x, t, \eta)-\varphi_{t}^{\prime}\left(x, t, \eta^{\prime}\right)\right| \geqslant c_{0} \lambda 2^{r} \lambda^{1 / 2} & \text { if } 2^{r} \leqslant \lambda^{\varepsilon_{0}}, \\
\left|\varphi_{t}^{\prime}(x, t, \eta)-\varphi_{t}^{\prime}\left(x, t, \eta^{\prime}\right)\right| \geqslant c_{0} \lambda 2^{r} \lambda^{1 / 2-\varepsilon_{0}} & \text { if } 2^{r} \geqslant \lambda^{\varepsilon_{0}},
\end{array}
$$

provided $C_{1}$ is chosen large enough, independent of $\lambda$ and $r$. Now the proof is finished by integration by parts similar as in the proof of Lemma 6.6. Note that one could have proved Lemma 6.6 above by performing integrations by parts in the $t$ variable alone.

Using Lemma 6.9 we see that the last two terms in (6.55) are bounded by $C_{\varepsilon, N} \lambda^{-N}\|f\|_{1}^{2}$ for every $N$. If we now apply the Schwarz inequality with respect to the $t$ variable we obtain

$$
\begin{aligned}
\left\|\mathscr{F}_{\lambda} f\right\|_{L^{4}\left(L^{2}\right)} \leqslant & C_{\varepsilon} \lambda^{\varepsilon}\left\|\left(\sum_{\nu}\left|\mathscr{F}_{\lambda}^{\nu} f\right|^{2}\right)^{1 / 2}\right\|_{L^{4}\left(L^{2}\right)}+C_{\varepsilon, N} \lambda^{-N}\|f\|_{4} \\
& +C_{\varepsilon} \sum_{\lambda^{\varepsilon} \leqslant 2^{r} \leqslant \lambda^{1 / 4}}\left(I_{r}\right)^{1 / 4}+C_{\varepsilon} \sum_{\lambda^{1 / 4} \leqslant 2^{r} \leqslant \lambda^{1 / 2-\varepsilon}}\left(I I_{r}\right)^{1 / 4}
\end{aligned}
$$

where

$$
\begin{aligned}
& I_{r}=\int \mid \sum_{\left|j-j^{\prime}\right| \leqslant C_{1} \mid} \sum_{\substack{\left|\nu-\nu^{\prime}\right| \approx 2^{r} \\
\nu^{\prime} \leqslant \nu}} \widetilde{\mathscr{F}}_{\lambda, r}^{\nu, j} f(x, t){\overline{\widetilde{F}_{\lambda, r}{ }^{\prime}, j^{\prime}} f(x, t)}_{\mid}^{2} d x d t \\
& =\int \sum_{\left|j-j^{\prime}\right| \leqslant C_{1}} \sum_{\left|k-k^{\prime}\right| \leqslant C_{1}} \sum_{\substack{\left|\nu-\nu^{\prime}\right| \approx 2^{r} \\
\nu^{\prime} \leqslant \nu}} \sum_{\substack{\left|\mu-\mu^{\prime}\right| \approx 2^{2} \\
\mu \leqslant \mu^{\prime}}} \widetilde{\mathscr{F}}_{\lambda, r}^{\nu, j} f(x, t) \\
& \times \widetilde{\mathscr{F}}_{\lambda, r}^{\mu, k} f(x, t){\overline{\widetilde{F}_{\lambda, r}^{\nu^{\prime}, j^{\prime}}} f(x, t) \widetilde{\mathscr{F}}_{\lambda, r}^{\mu^{\prime}, k^{\prime}} f(x, t)}_{d x} d t
\end{aligned}
$$


and, similarly,

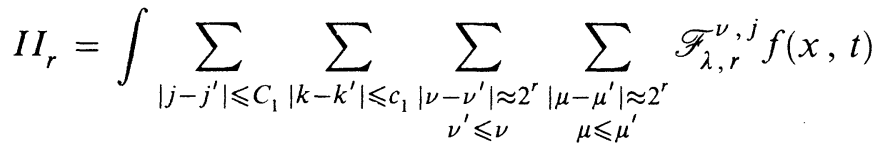

$$
\begin{aligned}
& \times \mathscr{F}_{\lambda, r}^{\mu, k} f(x, t) \overline{\mathscr{F}_{\lambda, r}^{\nu^{\prime}, j^{\prime}} f(x, t) \mathscr{F}_{\lambda, r}^{\mu^{\prime}, k^{\prime}} f(x, t)} d x d t .
\end{aligned}
$$

We estimated the first term in the right-hand side of (6.58) in the proof of Theorem 6.1. In order to estimate the terms $I_{r}$ and $I I_{r}$ we have to use another orthogonality estimate, which is similar to Lemma 6.7 .

Lemma 6.10. Suppose that $\left|\nu-\nu^{\prime}\right| \approx 2^{r},\left|\mu-\mu^{\prime}\right| \approx 2^{r}, \nu^{\prime} \leqslant \nu, \mu \leqslant \mu^{\prime}$, $\left|j-j^{\prime}\right| \leqslant c_{1},\left|k-k^{\prime}\right| \leqslant c_{1}$, and $\lambda>A_{1}$ for sufficiently large $A_{1}$. Furthermore assume that $|\nu-\mu|+\left|\nu^{\prime}-\mu^{\prime}\right| \geqslant A_{2}$ and $\left|\mu-\nu^{\prime}\right|+\left|\nu-\mu^{\prime}\right| \geqslant A_{2}$ for a sufficiently large constant $A_{2}$, independent of $\lambda$.

If $\lambda^{\varepsilon} \leqslant 2^{r} \leqslant \lambda^{1 / 4}$ and if $\nu<\mu$ or $\mu^{\prime}<\nu^{\prime}$ then

$$
\begin{aligned}
& \left|\int \widetilde{\mathscr{F}}_{\lambda, r}^{\nu, j} f(x, t) \widetilde{\mathscr{F}}_{\lambda, r}^{\mu, k} f(x, t){\overline{\widetilde{F}_{\lambda, r}{ }^{\prime}, j^{\prime}} f(x, t) \widetilde{\mathscr{F}}_{\lambda, r}^{\mu^{\prime}, k^{\prime}} f(x, t)}_{d x} d t\right| \\
& \leqslant C_{N} \lambda^{4} 2^{4 r}\left(2^{r}|\nu-\mu|+2^{r}\left|\nu^{\prime}-\mu^{\prime}\right|\right)^{-N}\|f\|_{1}^{4} \text {. }
\end{aligned}
$$

If $\lambda^{1 / 4} \leqslant 2^{r} \leqslant \lambda^{1 / 2-\varepsilon}$ and if $\mu<\nu$ and $\nu^{\prime}<\mu^{\prime}$ then

$$
\begin{aligned}
& \left|\int \mathscr{F}_{\lambda, r}^{\nu, j} f(x, t) \mathscr{F}_{\lambda, r}^{\mu, k} f(x, t){\overline{\mathscr{F}_{\lambda, r}^{\nu^{\prime}, j^{\prime}}} f(x, t) \mathscr{F}_{\lambda, r}^{\mu^{\prime}, k^{\prime}} f(x, t)}_{d x} d t\right| \\
& \leqslant C_{N} \lambda^{6} 2^{-4 r}\left(2^{r}\left|\nu-\mu^{\prime}\right|+2^{r}\left|\mu-\nu^{\prime}\right|\right)^{-N}\|f\|_{1}^{4} \text {. }
\end{aligned}
$$

If $\lambda^{\varepsilon} \leqslant 2^{r} \leqslant \lambda^{1 / 4}$ and if $\mu<\nu$ and $\nu^{\prime}<\mu^{\prime}$ then

$$
\begin{aligned}
& \left|\int \widetilde{\mathscr{F}}_{\lambda, r}^{\nu, j} f(x, t) \underset{\lambda, r}{\widetilde{\mathscr{F}}_{\lambda, k}^{\mu} f(x, t) \overline{\widetilde{F}}_{\lambda, r}^{\nu^{\prime}, j^{\prime}} f(x, t) \widetilde{\mathscr{F}}_{\lambda, r}^{\mu^{\prime}, k^{\prime}} f(x, t)} d x d t\right| \\
& \leqslant C_{N} \lambda^{4} 2^{4 r}\left(2^{r}\left|\nu-\mu^{\prime}\right|+2^{r}\left|\mu-\nu^{\prime}\right|\right)^{-N}\|f\|_{1}^{4} \text {. }
\end{aligned}
$$

If $\lambda^{1 / 4} \leqslant 2^{r} \leqslant \lambda^{1 / 2-\varepsilon}$ and if $\nu<\mu$ or $\mu^{\prime}<\nu^{\prime}$ then

$$
\begin{aligned}
& \left|\int \mathscr{F}_{\lambda, r}^{\nu, j} f(x, t) \mathscr{F}_{\lambda, r}^{\mu, k} f(x, t) \overline{\mathscr{F}_{\lambda, r}^{\nu^{\prime}, j^{\prime}} f(x, t) \mathscr{F}_{\lambda, r}^{\mu^{\prime}, k^{\prime}} f(x, t)} d x d t\right| \\
& \leqslant C_{N} \lambda^{6} 2^{-4 r}\left(2^{r}|\nu-\mu|+2^{r}\left|\nu^{\prime}-\mu^{\prime}\right|\right)^{-N}\|f\|_{1}^{4} \text {. }
\end{aligned}
$$

Let us remark that $(6.61)$ and (6.62) actually hold if $\lambda^{\varepsilon} \leqslant 2^{r} \leqslant \lambda^{1 / 2-\varepsilon}$. On the other hand, the restrictions on $r$ in (6.63), (6.64) are necessary, and this is the reason why we had to introduce two different decompositions $\mathscr{F}_{\lambda, r}{ }^{\nu, j}$ and $\widetilde{F}_{\lambda, j}^{\nu, j}$.

Given Lemma 6.10 straightforward applications of the Schwarz inequality 
show that

$$
\begin{aligned}
I_{r} \leqslant & C_{\varepsilon} \lambda^{4 \varepsilon}\left\|\left(\sum_{\nu, j}\left|\widetilde{F}_{\lambda, r}^{\nu, j} f\right|^{2}\right)^{1 / 2}\right\|_{L^{4}\left(\mathbb{R}^{3}\right)}^{4} \\
& +C_{\varepsilon, N} \lambda^{-N}\|f\|_{L^{4}\left(\mathbb{R}^{2}\right)}^{4} \text { if } \lambda^{\varepsilon} \leqslant 2^{r} \leqslant \lambda^{1 / 4} \\
I I_{r} \leqslant & C_{\varepsilon} \lambda^{4 \varepsilon}\left\|\left(\sum_{\nu, j}\left|\mathscr{F}_{\lambda, r}^{\nu, j} f\right|^{2}\right)^{1 / 2}\right\|_{L^{4}\left(\mathbb{R}^{3}\right)}^{4} \\
& +C_{\varepsilon, N} \lambda^{-N}\|f\|_{L^{4}\left(\mathbb{R}^{2}\right)}^{4} \text { if } \lambda^{1 / 4} \leqslant 2^{r} \leqslant \lambda^{1 / 2-\varepsilon}
\end{aligned}
$$

and, since $\varepsilon$ can be chosen arbitrary small, this together with (6.58) proves (6.47).

Proof of Lemma 6.10. We use the same integration by parts argument as in the proof of Lemma 6.7. Now let $b_{j, k, j^{\prime}, k^{\prime}}^{\nu, \mu, \nu^{\prime}}$ be as in (6.18) with $\ell$ replaced by $r$, and, analogously, let

$$
\begin{aligned}
& \tilde{b}_{j, k, j^{\prime}, k^{\prime}}^{\nu, \mu, \nu^{\prime}, \mu^{\prime}}\left(x, t, \eta, \xi, \eta^{\prime}, \xi^{\prime}\right) \\
& \quad=\tilde{a}_{\lambda, r}^{\nu, j}(x, t, \eta) \tilde{a}_{\lambda, r}^{\mu, k}(x, t, \xi) \overline{\tilde{a}_{\lambda, r}^{\nu^{\prime}, j^{\prime}}\left(x, t, \eta^{\prime}\right) \tilde{a}_{\lambda, r}^{\mu^{\prime}, k^{\prime}}\left(x, t, \xi^{\prime}\right)} .
\end{aligned}
$$

Observe that derivatives of $\tilde{b}$ behave much better then the derivatives of $b$, namely

$$
\begin{aligned}
& \left|\partial_{x, t}^{\alpha} \tilde{b}_{j, k, j^{\prime}, k^{\prime}}^{\nu, \mu, \nu^{\prime}}\left(x, t, \eta, \xi, \eta^{\prime}, \xi^{\prime}\right)\right| \leqslant C_{\alpha}, \\
& \left|\partial_{x, t}^{\alpha} b_{j, k, j^{\prime}, k^{\prime}}^{\nu, \mu, \nu^{\prime}, \mu^{\prime}}\left(x, t, \eta, \xi, \eta^{\prime}, \xi^{\prime}\right)\right| \leqslant C_{\alpha} 2^{r|\alpha|} .
\end{aligned}
$$

The various terms that come up if we perform the integration by parts (cf. (6.20)) are evaluated using Lemma 6.8. In all cases we have to check that assumptions (5) and (6) of that lemma are satisfied in our context. Let us assume that $|\nu-\mu|+\left|\nu^{\prime}-\mu^{\prime}\right| \approx 2^{\ell}$.

In order to prove (6.61) we have to apply (6.22) and (6.23) with respect to the variables $\varphi_{x}^{\prime}(x, t, \eta)$. For fixed $x, t$ we then set $h(\eta)=\left|J_{x, t}(\eta)\right|$, where $J_{x, t}$ is the inverse to the homogeneous diffeomorphism $\eta \rightarrow \varphi_{x}^{\prime}(x, t, \eta)$. Suppose that $\eta \in \operatorname{supp} \tilde{a}_{\lambda, r}^{\nu, j}$ and $\eta^{\prime} \in \operatorname{supp} \tilde{a}_{\lambda, r}^{\nu^{\prime}, j^{\prime}}$. Then for fixed $x, t$

$$
\left|h\left(\varphi_{x}^{\prime}(\eta)\right)-h\left(\varphi_{x}^{\prime}\left(\eta^{\prime}\right)\right)\right|=|| \eta|-| \eta^{\prime}|| \leqslant C 2^{r} \lambda^{1 / 2-\varepsilon_{0}} .
$$

To apply Lemma 6.8 we define $n$ by letting $2^{-n} \lambda$ equal the right-hand side. Then since $\left|\nu-\nu^{\prime}\right|+\left|\mu-\mu^{\prime}\right| \approx 2^{r}$, we see that $2^{\ell-n} \leqslant C 2^{r} 2^{\ell} \lambda^{-1 / 2-\varepsilon_{0}} \leqslant$ $a_{0}^{-1}\left(\left|\nu-\nu^{\prime}\right|+\left|\mu-\mu^{\prime}\right|\right)$. The same argument applies to $\left|h\left(\varphi_{x}^{\prime}(\xi)\right)-h\left(\varphi_{x}^{\prime}\left(\xi^{\prime}\right)\right)\right|$, and therefore we may use Lemma 6.8. We perform the integration by parts and 
use also (6.65) to get

$$
\begin{aligned}
& \left|\int \widetilde{\mathscr{F}}_{\lambda, r}^{\nu, j} f(x, t) \widetilde{\mathscr{F}}_{\lambda, r}^{\mu, k} f(x, t) \overline{\widetilde{F}}_{\lambda, r}^{\nu^{\prime}, j^{\prime}} f(x, t) \widetilde{\mathscr{F}}_{\lambda, r}^{\mu^{\prime}, k^{\prime}} f(x, t) d x d t\right| \\
& \quad \leqslant C_{\varepsilon, N} \lambda^{4} 2^{4 r}\left(\left|\nu-\nu^{\prime}\right|\left(2^{\ell}+\left|\nu-\nu^{\prime}\right|\right)+\left|\mu-\mu^{\prime}\right|\left(2^{\ell}+\left|\mu-\mu^{\prime}\right|\right)\right)^{-N}\|f\|_{1}^{4} \\
& \quad \leqslant \lambda^{4} 2^{4 r}\left(2^{r}|\nu-\mu|+2^{r}\left|\nu^{\prime}-\mu^{\prime}\right|\right)^{-N}\|f\|_{1}^{4} .
\end{aligned}
$$

In order to see (6.62) assume $\mu<\nu$ and $\nu^{\prime}<\mu^{\prime}$; that is, we have one of the following cases: $\mu<\nu^{\prime}<\nu<\mu^{\prime}, \mu<\nu^{\prime}<\mu^{\prime}<\nu, \nu^{\prime}<\mu<\mu^{\prime}<\nu$, or $\nu^{\prime}<\mu<\nu<\mu^{\prime}$. In either one of these cases we notice that $2^{\ell} \approx 2^{r}$. We want to apply Lemma 6.8 with $h=q$. Now if $\eta \in \operatorname{supp} a_{\lambda, r}^{\nu, j}$ and $\eta^{\prime} \in \operatorname{supp} a_{\lambda, r}^{\nu^{\prime}, j^{\prime}}$, then

$$
\left|q\left(\varphi_{x}^{\prime}(x, t, \eta)\right)-q\left(\varphi_{x}^{\prime}\left(x, t, \eta^{\prime}\right)\right)\right| \leqslant C \lambda 2^{-r} .
$$

So if we let $\lambda 2^{-n}$ be the right-hand side, we have $2^{\ell-n} \leqslant C$ and, hence, we may apply (6.24) and (6.25) to obtain

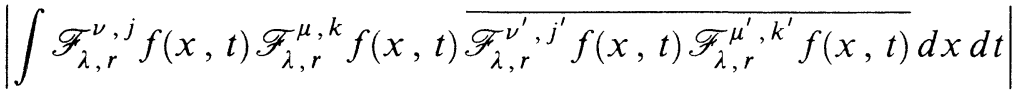

$$
\begin{aligned}
& \leqslant C_{\varepsilon, N} \lambda^{6} 2^{-4 r}\left(\left|\mu-\nu^{\prime}\right|\left(2^{\ell}+\left|\mu-\nu^{\prime}\right|\right)+\left|\nu-\mu^{\prime}\right|\left(2^{\ell}+\left|\nu-\mu^{\prime}\right|\right)\right)^{-N}\|f\|_{1}^{4} .
\end{aligned}
$$

This implies (6.62) since now $2^{r} \approx 2^{\ell}$.

In order to see (6.63) we note that in this case again $2^{r} \approx 2^{\ell}$. With $n$ as in (6.67) we have $2^{\ell-n} \leqslant C 2^{2 r} \lambda^{-1 / 2-\varepsilon_{0}} \leqslant C^{\prime}$ since now $2^{r} \leqslant \lambda^{1 / 4}$. To obtain (6.64) we need to observe that if, as in (6.68), $n=r$, then $2^{\ell-n} \leqslant 2^{r} \approx\left|\nu-\nu^{\prime}\right|+\left|\mu-\mu^{\prime}\right|$, since now $2^{2 r} \geqslant \lambda^{1 / 2} \geqslant 2^{\ell}$.

\section{MaXimal Riesz MEANS, MUltiplier tRansformations, AND OSCILLATORY INTEGRALS}

Let $(M, g)$ be a compact Riemannian manifold of dimension 2 , and let $\Delta$ be the Laplace-Beltrami operator. We shall assume that $(M, g)$ satisfies the following

Periodicity Assumption: The geodesic flow is periodic with minimal period $\Pi$.

We consider potentials $V$ such that $-\Delta+V$ is a positive operator. We shall study $L^{p}$ boundedness of Riesz means, defined in (1.4), and of the associated maximal operators.

Theorem 7.1. Suppose that $(M, g)$ satisfies the periodicity assumption (7.1). Let

$$
S_{*}^{\delta} f(x)=\sup _{R>0}\left|S_{R}^{\delta} f(x)\right|,
$$

and assume $2 \leqslant p<\infty$ and $\delta>\max \{2(1 / 2-1 / p)-1 / 2,0\}$. Then $S_{*}^{\delta}$ is a bounded operator on $L^{p}(M)$.

Remark. Our proof will show that more generally $S_{*}^{\delta}$ is a bounded operator from the Sobolev space $L_{\alpha}^{p}(M)$ into $L^{p}(M)$ if $\alpha, \delta \geqslant 0$, and $\delta+\alpha>$ 
$\max \{2(1 / 2-1 / p)-1 / 2,0\}$. In particular, if $S_{R}=\sum_{k \leqslant R} E_{k}$ is the partial summation operator then we have for $f \in L_{\alpha}^{p}, 2 \leqslant p \leqslant 4, \alpha>0$

$$
\left(\int \sup _{R \geqslant \lambda}\left|S_{R} f(x)-f(x)\right|^{p} d x\right)^{1 / p} \rightarrow 0 \quad \text { as } \lambda \rightarrow \infty .
$$

This convergence result is trivially true for smooth functions, and it follows for $f \in L_{\alpha}^{p}$ by approximating $f$ by smooth functions and applying the maximal inequality. Similarly one deduces the summability result for Riesz means, mentioned in the introduction.

We consider also more general multiplier operators $T_{m}$, which are defined for bounded continuous functions $m$ by

$$
T_{m} f(x)=\sum_{k} m(k) E_{k} f .
$$

We shall assume the usual Hörmander condition in one dimension, namely,

$$
\sup _{s>0}\|\beta(\cdot) m(s \cdot)\|_{L_{\alpha}^{2}(\mathbb{R})}=A_{\alpha}<\infty
$$

for some $\alpha>1 / 2$. Here $\beta \in \mathscr{C}_{0}^{\infty}(\mathbb{R})$ is supported in the interval $(1 / 2,2)$ and positive in a compact subinterval. The following result generalizes the multiplier theorem in [8].

Theorem 7.2. Suppose that $(M, g)$ satisfies the periodicity assumption (7.1). Assume that $1<p<\infty$ and $\alpha>\max \{2|1 / 2-1 / p|, 1 / 2\}$ and (7.3) is satisfied. Then

$$
\left\|T_{m} f\right\|_{p} \leqslant C A_{\alpha}\|f\|_{p} .
$$

In the proofs of our results it will be convenient to consider functions of $\sqrt{-\Delta+V}$ rather than $-\Delta+V$. This makes no difference since one can easily check that for a given $m$ the left-hand side of (7.3) is bounded if and only if this is true for $m(\sqrt{\cdot})$. Therefore, we shall consider a classical selfadjoint positive first-order pseudodifferential operator $A$, and we shall assume that the subprincipal symbol, $\operatorname{sub}(A)$, of $A$ is constant. Moreover, if

$$
H_{a}=\sum\left(\frac{\partial a}{\partial x_{j}} \frac{\partial}{\partial \xi_{j}}-\frac{\partial a}{\partial \xi_{j}} \frac{\partial}{\partial x_{j}}\right)
$$

is the Hamiltonian vector field, we shall assume the analogue of (7.1), namely,

Periodicity Assumption: The Hamiltonian flow $\exp t H_{a}$ is periodic with minimal period $\Pi$.

Then, according to results of Weinstein [39] and Colin de Verdière [11], there are constants $c, C \geqslant 0$ such that eigenvalues $\mu_{j}$ of $A$ are for $\mu_{j}>C$ contained in the union of the intervals $\left[\gamma_{k}-c / k, \gamma_{k}+c / k\right]$ for suitable $c \geqslant 0$. Here

$$
\gamma_{k}=\frac{2 \pi}{\Pi}\left(k+\frac{\sigma}{4}\right)
$$

where $\sigma=\operatorname{sub}(\mathrm{A})+\kappa$ and $-\kappa$ is the Maslov-Arnold index considered in $[14, \S 3]$. An important ingredient in the proof of these eigenvalue estimates is 
that one can slightly perturb $A$ to another pseudodifferential operator $B$ such that the wave operator $e^{i t B}$ is periodic (cf. [11, 20; Chapter XXIX]). More precisely, there is a selfadjoint first-order operator $B$ which commutes with $A$ such that $A-B$ is of order -1 and such that

$$
e^{i \Pi B}=e^{-i \sigma \pi / 2} \mathrm{Id} \text {. }
$$

The main step in the proofs of our results is an estimate for multipliers supported in dyadic intervals.

Proposition 7.3. Suppose $B$ is as in (7.5) and suppose that the level sets of the principal symbol, $\{\xi: a(x, \xi)=1\} \subset T_{x}^{*} M \backslash 0$, have nonvanishing Gaussian curvature. Let $m \in L_{\alpha}^{2}(\mathbb{R})$ be supported in the interval $(1,2)$, and assume that $\alpha>1 / 2$. Then we have for $2 \leqslant p \leqslant 4$

$$
\left\|\sup _{2^{j} \leqslant \tau \leqslant 2^{j+1}}|m(B / \tau) f|\right\|_{p} \leqslant C\|m\|_{L_{\alpha}^{2}}\|f\|_{p} .
$$

Proof. Let $\beta \in C_{0}^{\infty}((1 / 2,8))$ satisfy $\beta(s)=1$ for $1 \leqslant s \leqslant 4$ and put $L_{j} f=$ $\beta\left(B / 2^{j}\right) f$. Then

$$
m(B / \tau) f=\frac{1}{2 \pi} \int \tau \widehat{m}(\tau t) e^{i t B} L_{j} f d t, \quad \tau \in\left[2^{j}, 2^{j+1}\right] .
$$

By applying Schwarz's inequality we get

$$
\begin{aligned}
|m(B / \tau) f(x)| \leqslant & C\left(\int \tau\left(1+|\tau t|^{2}\right)^{\alpha}|\widehat{m}(\tau t)|^{2} d t\right)^{1 / 2} \\
& \times\left(\int \frac{\tau}{\left(1+|\tau t|^{2}\right)^{\alpha}}\left|e^{i t B} L_{j} f(x)\right|^{2} d t\right)^{1 / 2} \\
\leqslant & C\|m\|_{L_{\alpha}^{2}}\left(\int \frac{2^{j}}{\left(1+\left|2^{j} t\right|^{2}\right)^{\alpha}}\left|e^{i t B} L_{j} f(x)\right|^{2} d t\right)^{1 / 2} .
\end{aligned}
$$

Therefore, it suffices to prove the inequality

$$
\left\|\left(\int_{-\infty}^{\infty} \frac{2^{j}}{\left(1+\left|2^{j} t\right|^{2}\right)^{\alpha}}\left|e^{i t B} L_{j} f\right|^{2} d t\right)^{1 / 2}\right\|_{p} \leqslant C\|f\|_{p} .
$$

If we recall (7.5), then, since $\alpha>1 / 2$, we conclude that this in turn would be a consequence of

$$
\left\|\left(\int_{0}^{\Pi} \frac{2^{j}}{\left(1+\left|2^{j} t\right|^{2}\right)^{\alpha}}\left|e^{i t B} L_{j} f\right|^{2} d t\right)^{1 / 2}\right\|_{p} \leqslant C\|f\|_{p} .
$$

This would follow from

Lemma 7.4. Let $\varepsilon>0$. Then if $0<\gamma<\Pi / 2$ and $2 \leqslant p \leqslant 4$

$$
\left\|\left(\int_{\gamma}^{2 \gamma}\left|e^{i t B} L_{j} f\right|^{2} \frac{d t}{t}\right)^{1 / 2}\right\|_{L^{p}} \leqslant C_{\varepsilon}\left(1+\gamma 2^{j}\right)^{\varepsilon}\|f\|_{L^{p}} .
$$

Proof. If $\gamma$ is larger than a fixed constant, the estimate follows from Theorem 6.2 since $\left\|L_{j} f\right\|_{L_{\varepsilon}^{p}} \leqslant C 2^{j \varepsilon}\|f\|_{L^{p}}$. If $\gamma$ is less than $2^{-j}$, the estimate holds 
trivially as then $e^{i t B} L_{j}$ is bounded on all $L^{p}$ spaces since $\lambda \rightarrow e^{i t \lambda} \beta\left(\lambda / 2^{j}\right)$ belongs to a bounded subset of $S^{0}$ for $t<2^{-j}$. So, without loss of generality, we may assume that $2^{-j}<\gamma<\varepsilon_{0}$ where $\varepsilon_{0}$ is small.

In proving (7.7) we may assume that $f$ is supported in a small coordinate patch. Then if $t$ is small and we if we use local coordinates, we can write

$$
\left(e^{i t B} L_{j} f\right)(x)=\int e^{i \varphi(x, t, \eta)} b_{j}(x, t, \eta) \hat{f}(\eta) d \eta
$$

modulo an operator $R_{j}$ whose kernel is uniformly bounded. Here $b_{j} \in S^{0}$ vanishes unless $|\eta| \approx 2^{j}$ and $\varphi$ is a generating function for $\mathscr{C}$, the canonical relation for $e^{i t B}$. By the latter, we mean that (for small $t$ )

$$
\mathscr{C}=\left\{\left(x, t, \varphi_{x}^{\prime}(x, t, \eta), \varphi_{t}^{\prime}(x, t, \eta), \varphi_{\eta}^{\prime}(x, t, \eta), \eta\right)\right\} .
$$

Thus, since $e^{i t B}=$ Identity when $t=0$ and since the fact that $\tau=a(x, \xi)$ in $\mathscr{C}$ implies $\varphi_{t}^{\prime}(x, t, \eta)=a\left(x, \varphi_{x}^{\prime}(x, t, \eta)\right)$, we conclude that

$$
\varphi(x, t, \eta)=\langle x, \eta\rangle+t a(x, \eta)+t^{2} r(x, t, \eta),
$$

with $r \in S^{1}$ being homogeneous of degree one in $\eta$. Writing things in this way, we find, after making a change of scale, that (7.7) holds if and only if

$$
\begin{aligned}
& \left\|\left(\int_{1}^{2}\left|\int e^{i\left[\langle x, \eta\rangle+t a(\gamma x, \eta)+\gamma t^{2} r(\gamma x, \gamma t, \eta)\right]} b_{j}(\gamma x, \gamma t, \eta / \gamma) \hat{f}(\eta) d \eta\right|^{2} d t\right)^{1 / 2}\right\|_{p} \\
& \quad \leqslant C_{\varepsilon}\left(2^{j} \gamma\right)^{\varepsilon}\|f\|_{p}
\end{aligned}
$$

but this just follows from Theorem 6.2 as $b_{j}(\gamma x, \gamma t, \eta / \gamma) \in S^{0}$ vanishes for $|\eta| \geqslant C 2^{j} \gamma$.

Proof of Theorem 7.1. Since the result for $p=\infty$ is just the uniform $L^{\infty}$ boundedness of $S_{R}^{\delta}, \delta>1 / 2$ (see [31]) we have to prove the inequality only for $2 \leqslant p \leqslant 4$. We split $\left(1-s^{2}\right)_{+}^{\delta}=\widetilde{m}(s)+m(s)$ where $\widetilde{m} \in C_{0}^{\infty}$ vanishes for $s>1 / 2$. Using the calculus of pseudodifferential operators it is easy to see that the maximal function $\sup _{\tau}|\tilde{m}((-\Delta+V) / \tau) f|$ is controlled by a variant of the Hardy-Littlewood maximal function and, therefore, defines a bounded operator on $L^{p}$ if $1<p \leqslant \infty$.

We now let $A=\sqrt{-\Delta+V}$ and recall that the subprincipal symbol of $A$ vanishes. We find $B$ commuting with $A$, as in (7.5), the eigenvalues of $B$ are then contained in the arithmetic progression $\left\{\gamma_{k}\right\}$, which incidentally coincides with the arithmetic progression $\left\{\sigma_{k}\right\}$ in the introduction. Therefore, for large $\tau$ we see that

$$
\sum_{k} m(k / \tau) E_{k}=m(B / \tau)
$$


Now we can apply Proposition 7.3 and Littlewood-Paley theory and obtain

$$
\begin{aligned}
\left\|\sup _{\tau}|m(B / \tau) f|\right\|_{p} & \leqslant\left(\sum_{j}\left\|\sup _{1 / 2 \leqslant s \leqslant 2}\left|m\left(2^{-j} s B\right) L_{j} f\right|\right\|_{p}^{p}\right)^{1 / p} \\
& \leqslant C\left(\sum_{j}\left\|L_{j} f\right\|_{p}^{p}\right)^{1 / p} \\
& \leqslant C^{\prime}\left\|\left(\sum_{j}\left|L_{j} f\right|^{2}\right)^{1 / 2}\right\|_{p} \leqslant C^{\prime \prime}\|f\|_{p} .
\end{aligned}
$$

Proof of Theorem 7.2. Again it is sufficient to prove the $L^{p}$ boundedness of the operator $m(B), 2 \leqslant p \leqslant 4$, where $B$ is as in the proof of Proposition 7.3. We may split $m=\sum_{j>0} m_{j}\left(2^{-j} \cdot\right)$ where $m_{0}(s)$ vanishes for large $s$ and where $m_{j}$ is supported in $(1 / 2,2)$; then $m_{j} \in L_{\alpha}^{2}$, uniformly in $j$. In particular $m_{j} \in C^{\varepsilon}$ where $0<\varepsilon<\alpha-1 / 2$, uniformly in $j$, and by Proposition 7.3 we know that $m_{j}(B / \tau)$ is a bounded operator on $L^{p}$ with bounds uniformly in $\tau, j$. According to a result in [26] these two facts imply the $L^{q}$ boundedness of $m(B / \tau)$ for $2 \leqslant q<p$ (for references concerning previous results in the Euclidean context due to Carbery and the second author see also [26]). This together with another interpolation finishes the proof.

Remark. If we set $I=[-T, T]$ then we cannot control the bounds in Theorem 6.2 if $T \rightarrow \infty$. That is why we have to assume the periodicity assumption above. Let us drop this assumption for a moment and assume that $M$ is a compact manifold of dimension two and $A$ is a selfadjoint positive firstorder pseudodifferential operator with principal symbol $a$, having the property that the level sets $\{\xi: a(x, \xi)=1\} \subset T_{x}^{*} M \backslash 0$ have nonvanishing Gaussian curvature. Our argument will show that there is a sharp $L^{4}$ bound for the approximate projection operator

$$
\chi_{\tau}(A) f=\sum_{j} \chi\left(\lambda_{j}-\tau\right) e_{j}(f)
$$

provided $\chi$ is of exponential type, i.e., its Fourier transform is compactly supported. In this case the $L^{4}$ operator norm of $\chi_{\tau}(A)$ is $O\left(\tau^{\varepsilon}\right)$ for any $\varepsilon>0$, as $\tau \rightarrow \infty$, and the maximal function $\sup _{\tau>1} \tau^{-\varepsilon}\left|\chi_{\tau}(A) f\right|$ defines a bounded operator on $L^{4}$.

Remark. It would be interesting to prove a result like Theorem 7.2 for the operator $T_{m}$ replaced by $m(-\Delta+V)$. Both operators coincide for spherical harmonics expansions on the sphere. In order to prove a sharp $L^{4}$ result one would have to understand better the fine structure of the eigenvalue clusters. At present we are able to prove the inequality

$$
\begin{aligned}
&\|m(-\Delta+V) f\|_{p} \leqslant C \sup _{s>0}\|\beta(\cdot) m(s \cdot)\|_{L_{\alpha}^{2}(\mathbb{R})}\|f\|_{p} \\
& \text { if } \alpha>2(1 / 2-1 / p), p \geqslant 14 / 3 .
\end{aligned}
$$


By the argument in the proof of Theorem 7.2 we can assume that $m=m_{0}\left(\tau^{-1} \cdot\right)$ for some $m_{0} \in L_{\alpha}^{2}$ supported in $(1,2)$. Moreover, a simple averaging argument shows that we can assume that for fixed $l \in \mathbb{N}$

$$
m_{0}(s)=m_{0, l}(s)=2^{-l(\alpha-1 / 2)} \beta(s) \sum_{\nu \in \mathbb{Z}} a_{\nu} \chi_{l, \nu}(s)
$$

where $2(1 / 2-1 / p)<\alpha, \chi \in C_{0}^{\infty}(0,1)$, and $\chi_{l, \nu}(s)=\chi\left(2^{l} s-\nu\right)$.

Let $A=\sqrt{-\Delta+V}$ and $B$ be as in Proposition 7.3. Since we have seen that the corresponding inequality for $m_{0, l}(B / \tau)$ is true for $p \geqslant 4$, we have to examine the difference $m_{0, l}(A / \tau)-m_{0, l}(B / \tau)$, and it suffices to prove

$$
\begin{aligned}
& \left\|m_{0, l}(A / \tau)-m_{0, l}(B / \tau)\right\|_{2} \\
& \quad \leqslant C 2^{-l[\alpha-2(1 / p-1 / 2)-1 / 2]}\left(\sum\left|a_{\nu}\right|^{2}\right)^{1 / 2}\|f\|_{p^{\prime}} \quad \text { if } p \geqslant 14 / 3 .
\end{aligned}
$$

Note that

$$
m_{0, l}(A / \tau)-m_{0, l}(B / \tau)=\sum_{j}\left[m_{0, l}\left(\mu_{j} / \tau\right)-m_{0, l}\left(\tilde{\mu}_{j} / \tau\right)\right] e_{j}(f)
$$

where $\left|\mu_{j}-\tilde{\mu}_{j}\right| \leqslant C / \tau$. Let $J^{\nu}=\left[2^{-l} \nu, 2^{-l}(\nu+1)\right]$ and $I^{k}=\left[\gamma_{k}-c / k, \gamma_{k}+\right.$ $c / k]$. Using orthogonality we see that

$$
\begin{aligned}
& \left\|m_{0, l}(A / \tau)-m_{0, l}(B / \tau)\right\|_{2} \\
& \leqslant C 2^{-l \alpha}\left(\sum_{k \approx \tau} \sum_{\nu}\left|a_{\nu}\right|_{\substack{s, s^{\prime} \in \\
I^{k} \cap J^{\nu}}}\left|\chi_{\nu, l}\left(2^{l} \tau^{-1} s\right)-\chi_{\nu, l}\left(2^{l} \tau^{-1} s^{\prime}\right)\right|^{2}\left\|E_{k} f\right\|_{2}^{2}\right)^{1 / 2} .
\end{aligned}
$$

We shall use the fact that for $2 \leqslant p \leqslant 6$ the projection operator $E_{k}$ is bounded from $L^{2} \rightarrow L^{p}$ with norm $\leqslant k^{1 / 4-1 / 2 p}$ (see [30]). In order to apply this, note that for fixed $\nu$, and for Hölder exponents $0 \leqslant \delta \leqslant 1$

$$
\begin{gathered}
\left(\sum_{\substack{k \approx \tau \\
I^{k} \cap s^{\prime} \in}} \sup _{\substack{I^{\nu}\\
}}\left|\chi_{\nu, l}\left(2^{l} \tau^{-1} s\right)-\chi_{\nu, l}\left(2^{l} \tau^{-1} s^{\prime}\right)\right|^{2}\right)^{1 / 2} \\
\leqslant C \max \left\{1,\left(2^{-l} \tau\right)^{1 / 2}\right\}\left(2^{l} / \tau\right)^{\delta} \tau^{-\delta} .
\end{gathered}
$$

We apply this with $\delta=1 / 2$ if $2^{l} \leqslant \tau$ and with $\delta=2(1 / 2-1 / p)-1 / 2$ if $2^{l}>\tau$. If we use the $L^{p^{\prime}} \rightarrow L^{2}$ bounds for the projection operator, we get for $2 \leqslant p \leqslant 6$

$$
\begin{aligned}
\left\|m_{0, l}(A / \tau)-m_{0, l}(B / \tau)\right\|_{2} \leqslant & C 2^{-l(\alpha-1 / 2)}\left(\sum_{\nu}\left|a_{\nu}\right|^{2}\right)^{1 / 2} \tau^{1 / 4-1 / 2 p} \\
& \times \max \left\{\tau^{-1 / 2},\left(2^{l} \tau^{-2}\right)^{2(1 / 2-1 / p)-1 / 2}\right\}\|f\|_{p^{\prime}},
\end{aligned}
$$

and therefore if $2^{l} \leqslant \tau$,

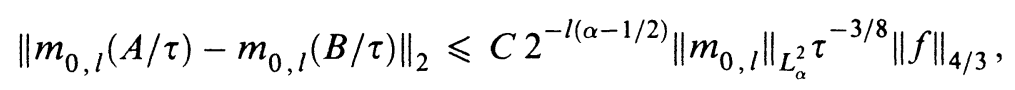


which is much better than what we wanted to prove. However, if $2^{l}>\tau$ we only get for $2 \leqslant p \leqslant 6$

$$
\left\|m_{0, l}(A / \tau)-m_{0, l}(B / \tau)\right\|_{2} \leqslant C 2^{-l\left(\alpha^{\prime}-2(1 / 2-1 / p)\right)}\left\|m_{0, l}\right\|_{L_{\alpha}^{2}} \tau^{7 / 2 p-3 / 4}\|f\|_{p^{\prime}},
$$

which is bounded independently of $\tau$ only if $p \geqslant 14 / 3$. This proves (7.9).

Maximal oscillatory integral theorems. Here we shall study oscillatory integral operators, which send functions of $n$ variables to functions of $n$ variables. As before we assume $n \geqslant 2$. Let

$$
S_{\lambda} f(x)=\int_{\mathbb{R}^{n}} e^{i \lambda \phi(x, y)} a(x, y) f(y) d y,
$$

where $a \in C_{0}^{\infty}\left(\mathbb{R}^{n} \times \mathbb{R}^{n}\right)$ and the real $C^{\infty}$ phase function $\phi$ satisfies a variant of the Carleson-Sjölin condition that we call the $n \times n$ Carleson-Sjölin condition. This requirement is that both $\nabla_{x} \phi$ and $\nabla_{y} \phi$ never vanish, that

$$
\operatorname{rank} \phi_{x y}^{\prime \prime} \equiv n-1 \text {, }
$$

and that there is a neighborhood $\mathcal{N}$ of $\operatorname{supp} a$ so that the immersed hypersurfaces

$$
\Sigma_{x_{0}}=\left\{\phi_{x}^{\prime}\left(x_{0}, y\right):\left(x_{0}, y\right) \in \mathcal{N}\right\} \subset T_{x_{0}}^{*} \mathbb{R}^{n} \backslash 0
$$

have everywhere nonvanishing Gaussian curvature.

These conditions arise naturally in the study of Fourier analysis on manifolds. For instance, $\phi(x, y)=\operatorname{dist}(x, y)$ satisfies the conditions near the diagonal, if $\operatorname{dist}(x, y)$ denotes the Riemannian distance between $x$ and $y$ with respect to some smooth metric $g$. For in this case

$$
\Sigma_{x_{0}}=\left\{\xi: \sum_{j, k} g^{j k}\left(x_{0}\right) \xi_{j} \xi_{k}=1\right\},
$$

and (7.11) is easy to check. Also, (7.11) and (7.12) are natural analogues of the conditions (2.17) and (2.20) for phase functions of nonhomogeneous oscillatory integral operators sending functions of $n-1$ variables into functions of $n$ variables. In fact, the latter can be used to prove estimates for the operators $S_{\lambda}$. This is because one can choose local coordinates $y=\left(y^{\prime}, y_{n}\right) \in \mathbb{R}^{n-1} \times \mathbb{R}$ so that for fixed $y_{n}$ the functions $\Phi\left(x, y^{\prime}\right)=\phi\left(x, y^{\prime}, y_{n}\right)$ satisfy the analogues of (2.17) and (2.20) in $n-1$ dimensions. So by using the oscillatory integral theorems in $[9,21,36]$ for such operators one can deduce the following sharp $L^{p} \rightarrow L^{q}$ mapping properties for the operators $S_{\lambda}$, namely, as $\lambda \rightarrow+\infty$,

$$
\left\|S_{\lambda} f\right\|_{L^{q}\left(\mathbb{R}^{n}\right)} \leqslant C \lambda^{-n / q}\|f\|_{L^{p}\left(\mathbb{R}^{n}\right)},
$$

if $q=\frac{n+1}{n-1} p^{\prime}$ and $1 \leqslant p \leqslant 2$ for $n \geqslant 3$ or $1 \leqslant p<4$ if $n=2$.

Using the square function estimates in Theorems 3.2 and 6.2 we can obtain a stronger maximal theorem for these operators. 
Theorem 7.5. Let $S_{\lambda}$ be as above. Then

(1) If $n \geqslant 2,1 \leqslant p \leqslant 2$, and $q=\frac{n+1}{n-1} p^{\prime}$,

$$
\left\|\sup _{\lambda>1}\left|\lambda^{n / q} S_{\lambda} f\right|\right\|_{L^{q}\left(\mathbb{R}^{n}\right)} \leqslant C\|f\|_{L^{p}\left(\mathbb{R}^{n}\right)}
$$

(2) If $n=2,2<p \leqslant 4, q=3 p^{\prime}$, and $\varepsilon>0$,

$$
\left\|\sup _{\lambda>1}\left|\lambda^{2 / q-\varepsilon} S_{\lambda} f\right|\right\|_{L^{q}\left(\mathbb{R}^{2}\right)} \leqslant C_{\varepsilon}\|f\|_{L^{p}\left(\mathbb{R}^{2}\right)} \text {. }
$$

This generalizes maximal theorems in $[7,10,25]$ concerning the model case where $\phi(x, y)=|x-y|$.

Proof. We shall give the proof of (1) and reduce it to an application of Theorem 3.2 for a suitable Fourier integral operator. The proof of (2) follows the same lines using instead Theorem 6.2 and is therefore omitted.

The inequality is trivial in the case $p=1$, so by interpolation it suffices to prove

$$
\left\|\sup _{\lambda>1}\left|\lambda^{n / q} S_{\lambda} f\right|\right\|_{L^{q}\left(\mathbb{R}^{n}\right)} \leqslant C\|f\|_{L^{2}\left(\mathbb{R}^{n}\right)}, \quad q=\frac{2(n+1)}{n-1} ;
$$

but if $\rho(\lambda) \in C^{\infty}\left(\mathbb{R}_{+}\right)$vanishes near 0 and equals one for $\lambda>1$, we argue as in $\S 5$ to get

$$
\begin{aligned}
\sup _{\lambda>1}\left|\lambda^{n / q} S_{\lambda} f(x)\right|^{2} \leqslant & \left(\int_{0}^{\infty}\left|\rho(\lambda) \lambda^{n / q} S_{\lambda} f(x)\right|^{2} d \lambda\right)^{1 / 2} \\
& \times\left(\int_{0}^{\infty}\left|\frac{d}{d \lambda}\left\{\rho(\lambda) \lambda^{n / q} S_{\lambda} f(x)\right\}\right|^{2} d \lambda\right)^{1 / 2} .
\end{aligned}
$$

Hence it suffices to show that for $q$ as in (7.13)

$$
\begin{gathered}
\left\|\left(\int_{0}^{\infty}\left|\rho(\lambda) \lambda^{n / q} S_{\lambda} f\right|^{2} d \lambda\right)^{1 / 2}\right\|_{L^{q}} \leqslant C\|f\|_{L^{2}}, \\
\left\|\left(\int_{0}^{\infty}\left|\frac{d}{d \lambda}\left\{\rho(\lambda) \lambda^{n / q} S_{\lambda} f\right\}\right|^{2} d \lambda\right)^{1 / 2}\right\|_{L^{q}} \leqslant C\|f\|_{L^{2}} .
\end{gathered}
$$

We shall only prove the first square function estimate, since the second follows from the same argument.

If we use Plancherel's theorem, we see that

$$
\int\left|\rho(\lambda) \lambda^{n / q} S_{\lambda} f(x)\right|^{2} d \lambda=\frac{1}{2 \pi} \int|\mathscr{F} f(x, t)|^{2} d t,
$$

where

$$
\mathscr{F} f(x, t)=\int e^{i \lambda[\phi(x, y)-t]} \rho(\lambda) \lambda^{n / q} a(x, y) f(y) d \lambda d y .
$$


Note that $\mathscr{F}$ is a Fourier integral operator of class $I^{-1 / 4+\mu}\left(\mathbb{R}^{n+1}, \mathbb{R}^{n} ; \mathscr{C}\right)$ where $\mu=n / q-(n-1) / 2$ and

$$
\mathscr{C}=\left\{\left(x, \phi(x, y), \lambda \phi_{x}^{\prime}(x, y), \lambda,-\lambda \phi_{y}^{\prime}(x, y)\right):(x, y) \in \operatorname{supp} a, \lambda \neq 0\right\} .
$$

It is easy to check that $\mathscr{C}$ satisfies conditions (2.2), (2.3), and (2.6)-see also the remark below.

Now if $T=2 \sup |\phi(x, y)|$ then an integration by parts in $\lambda$ leads to the estimate

$$
|\mathscr{F} f(x, t)| \leqslant C_{N}|t|^{-N}\|f\|_{L^{1}}, \quad|t|>T,
$$

while by Theorem 3.2

$$
\left\|\left(\int_{-T}^{T}|\mathscr{F} f(\cdot, t)|^{2} d t\right)^{1 / 2}\right\|_{L^{q}} \leqslant C\|f\|_{L^{2}} .
$$

This finishes the proof.

Remark. The conditions on the phase functions can be relaxed somewhat. Suppose that $\phi \in C^{\infty}$ is a real-valued phase function whose Monge-Ampere determinant is nonsingular:

$$
\operatorname{det}\left(\begin{array}{cc}
0 & \partial \phi / \partial x \\
\frac{\partial \phi}{\partial y} & \frac{\partial^{2} \phi}{\partial x \partial y}
\end{array}\right) \neq 0, \quad(x, y) \in \mathscr{N} .
$$

Then, of course, $\phi_{x}^{\prime} \neq 0, \phi_{y}^{\prime} \neq 0$ in $\mathscr{N}$. (7.15) also implies that, for every value of $t_{0}=\phi\left(x_{0}, y_{0}\right)$ with $\left(x_{0}, y_{0}\right) \in \mathscr{N}$, the set

$$
\Sigma_{x_{0}, t_{0}}=\left\{\phi_{x}^{\prime}\left(x_{0}, y\right):\left(x_{0}, y\right) \in \mathcal{N}, \phi\left(x_{0}, y\right)=t_{0}\right\} \subset T_{x_{0}}^{*} \mathbb{R}^{n} \backslash 0
$$

is an immersed hypersurface. Our third assumption is the curvature condition:

$$
\text { For }\left(x_{0}, t_{0}\right) \text { as above, the hypersurfaces } \Sigma_{x_{0}, t_{0}} \text { have everywhere }
$$

nonvanishing Gaussian curvature.

If $S_{\lambda}$ is defined as in (7.10) then the estimates in Theorem 3.4 remain valid if the phase function $\phi$ satisfies (7.15) and (7.16). To see this one has to check that the canonical relation $\mathscr{C}$ in (7.14) satisfies conditions (2.2), (2.3), and (2.6), but our assumption about the Monge-Ampere matrix of $\phi$ is equivalent to (2.3) and this assumption also gives us (2.2). Finally, in this setting (2.6) is equivalent to our curvature assumption (7.16).

\section{REFERENCES}

1. R. Beals, Spatially inhomogeneous pseudodifferential operators. II, Comm. Pure Appl. Math. 27 (1974), 161-205.

2. P. Bérard, Riesz means on Riemannian manifolds, Proc. Sympos. Pure Math., vol. 36, Amer. Math. Soc., Providence, RI, 1980, pp. 1-12.

3. A. Besse, Manifolds all of whose geodesics are closed, Springer-Verlag, Berlin, 1978.

4. J. Bourgain, Averages in the plane over convex curves and maximal operators, J. Anal. 47 (1986), 69-85. 
5. Funct. Anal. 1 (1991), 147-187.

6. P. Brenner, $L_{p}-L_{p^{\prime}}$-estimates for Fourier integral operators related to hyperbolic equations, Math. Z. 152 (1977), 273-286.

7. A. Carbery, The boundedness of the maximal Bochner-Riesz operator on $L^{4}\left(\mathbb{R}^{2}\right)$, Duke Math. J. 50 (1983), 409-416.

8. A. Carbery, G. Gasper, and W. Trebels, Radial Fourier multipliers of $L^{p}\left(\mathbb{R}^{2}\right)$, Proc. Nat. Acad. Sci. U.S.A. 81 (1984), 3254-3255.

9. L. Carleson and P. Sjölin, Oscillatory integrals and a multiplier problem for the disc, Studia Math. 44 (1972), 287-299.

10. M. Christ, On the almost everywhere convergence of Bochner-Riesz means in higher dimensions, Proc. Amer. Math. Soc. 95 (1985), 16-20.

11. Y. Colin de Verdière, Sur le spectra des opérateurs elliptiques à bicharactéristiques toutes périodiques, Comment. Math. Helv. 54 (1979), 508-522.

12. A. Córdoba, A note on Bochner-Riesz operators, Duke Math. J. 46 (1979), 505-511.

13. __ Geometric Fourier analysis, Ann. Inst. Fourier (Grenoble) 32 (1982), 215-226.

14. J. J. Duistermaat and V. W. Guillemin, The spectrum of positive elliptic operators and periodic bicharacteristics, Invent. Math. 29 (1975), 39-79.

15. C. Fefferman, A note on spherical summation multipliers, Israel J. Math. 15 (1973), 44-52.

16. J. Garcia-Cuerva and J. L. Rubio de Francia, Weighted norm inequalities and related topics, North-Holland, Amsterdam, 1985.

17. A. Greenleaf and G. Uhlmann, Estimates for singular Radon transforms and pseudodifferential operators with singular symbols, J. Funct. Anal. 89 (1990), 202-232.

18. L. Hörmander, The spectral function of an elliptic operator, Acta Math. 88 (1968), 341-370.

19. __ Fourier integral operators. I, Acta Math. 127 (1971), 79-183.

20. _ The analysis of linear partial differential operators, Vols. I-IV, Springer-Verlag, Berlin, 1983, 1985.

21. __ Oscillatory integrals and multipliers on $F L^{p}$, Ark. Mat. 11 (1971), 1-11.

22. J. -L. Journé, A. Soffer, and C. D. Sogge, Decay estimates for Schrödinger operators, Comm. Pure Appl. Math. 44 (1991), 573-604.

23. M. Kaneko and G. Sunouchi, On the Littlewood-Paley and Marcinkiewicz functions in higher dimensions, Tôhoku Math. J. 37 (1985), 343-365.

24. G. Mockenhaupt, A. Seeger, and C. D. Sogge, Wave front sets, local smoothing and Bourgain's circular maximal theorem, Ann. of Math. (2) 136 (1992), 207-218.

25. A. Seeger, On quasiradial Fourier multipliers and their maximal functions, J. Reine Angew. Math. 370 (1986), 61-73.

26. A. Seeger and C. D. Sogge, On the boundedness of functions of (pseudo)-differential operators on compact manifolds, Duke Math. J. 59 (1989), 709-736.

27. 669-682.

28. A. Seeger, C. D. Sogge and E. M. Stein, Regularity properties of Fourier integral operators, Ann. of Math. (2) 103 (1991), 231-251.

29. C. D. Sogge, Oscillatory integrals and spherical harmonics, Duke Math. J. 53 (1986), 43-65.

30. Concerning the $L^{p}$ norm of spectral clusters for second order elliptic operators on compact manifolds, J. Funct. Anal. 77 (1988), 123-134.

31. _ On the convergence of Riesz means on compact manifolds, Ann. of Math. (2) 126 (1987), 439-447.

32. __ Propagation of singularities and maximal functions in the plane, Invent. Math. 104 (1991), 349-376. 
33. C. D. Sogge and E. M. Stein, Averages over hypersurfaces. II, Invent. Math. 86 (1986), 233-242.

34. _ Averages of functions over hypersurfaces: Smoothness of generalized Radon transforms, J. Analyse Math. 54 (1990), 165-188.

35. E. M. Stein, Maximal functions: spherical means, Proc. Nat. Acad. Sci. U.S.A. 73 (1976), 2174-2175.

36. __ Oscillatory integrals in Fourier analysis, Beijing Lectures in Harmonic Analysis, Princeton Univ. Press, Princeton, NJ, 1986, pp. 307-356.

37. S. Sternberg, Lectures on differential geometry, Chelsea, New York, NY, 1964 and 1983.

38. R. Strichartz, A priori estimates for the wave equation and some applications, J. Funct. Anal. 5 (1970), 218-235.

39. __ Restriction of Fourier transform to quadratic surfaces, Duke Math. J. 44 (1977), 705-714.

40. A. Weinstein, Asymptotics of eigenvalue clusters for the Laplacian plus a potential, Duke Math. J. 44 (1977), 883-892.

(G. Mockenhaupt and C. D. Sogge) Department of Mathematics, University of CaliFORNIA, Los ANGELES, CALIFORNIA 90024

Current address, G. Mockenhaupt: Fachbereich Mathematik, Universität Siegen, 5900 Siegen, Germany

E-mail address, C. D. Sogge: sogge@math.ucla.edu

(A. Seeger) Department of Mathematics, Princeton University, Princeton, New JerSEY 08544

Current address: University of Wisconsin, Madison, Wisconsin 53706

E-mail address: seeger@math.wisc.edu 